\title{
Novel Solutions and Applications to Elevator-like Problems
}

\author{
By \\ Omar Ghaleb

\begin{abstract}
A thesis submitted to
the Faculty of Graduate and Postdoctoral Affairs

in partial fulfilment of

the requirements for the degree of

Master of Computer Science
\end{abstract} \\ Ottawa-Carleton Institute for Computer Science \\ School of Computer Science \\ Carleton University \\ Ottawa, Ontario
}

September 2018

(C) Copyright

2018, Omar Ghaleb 
The undersigned hereby recommend to the Faculty of Graduate and Postdoctoral Affairs acceptance of the thesis,

Novel Solutions and Applications to

\section{Elevator-like Problems}

submitted by

\section{Omar Ghaleb}

\begin{tabular}{c}
\hline Dr. Douglas Howe \\
(Director, School of Computer Science) \\
\hline $\begin{array}{c}\text { Dr. B. John Oommen } \\
\text { (Thesis Supervisor) }\end{array}$
\end{tabular}

(Thesis Supervisor)

Carleton University

September 2018 


\begin{abstract}
The field of AI has been a topic of interest for the better part of a century, where the goal is to have computers mimic human behaviour. Researchers have attempted to incorporate AI in different problem domains, such as autonomous driving, playing games like Chess and Go, diagnosis and security. They have also worked extensively on different subfields of AI such as machine learning, pattern recognition and voice operated-systems.

This thesis concentrates on a subfield of AI which is the field of Learning Automata (LA). Rather than working with the well-established mathematical formulations of the field, our intention has been to use these tools to tackle a specific set of problems referred to as Elevator-Like Problems.

Our work in this thesis considers a problem that has not been tackled before using AI. It involves the problem of optimizing the scheduling of elevators. In particular, we are concerned with determining the Elevators' optimal "parking" locations. Problems with similar characteristics are referred to as Elevator-like Problems (ELPs). In our case, the objective is to find the optimal parking floors for the set of available elevators so as to minimize the passengers' Average Waiting Time (AWT).

Apart from proposing benchmark solutions, we have provided two different novel LA-based solutions for two different general settings, namely the single-elevator and the multi-elevator scenarios. The first pair of solutions are based on the well-known $L_{R I}$ scheme, and the second pair incorporate the Pursuit concept to improve the performance and the convergence speed of the first solutions, leading to the $P L_{R I}$ scheme. The simulation results presented demonstrate that our solutions performed better than those used in modern-day elevators, and provided results that are nearoptimal, yielding a performance increase of up to $90 \%$.
\end{abstract}




\section{ACKNOWLEDGEMENTS}

I would like to primarily thank my Supervisor and Mentor, Prof. B. John Oommen, for his direction, encouragement and the significant advice and help that I received from him. During my studies, he was like a second father to me, and he treated me like his son. His door was always open for me no matter when I needed him. I would not have been able to complete such a research program and thesis without his help. There are not enough words that I can use to describe how thankful I am to have had him as a Supervisor.

I would like to offer a special thanks to my family for their support. My father, Amin, is my role model in life and is the one whom I am striving to be like. He supported me in my journey through life without any hesitation, and believed in me. I owe my mother, Eman, an indescribable debt. It was she who stood by my side, and who constantly encouraged me to go further, and who made it easier for me during all my trials in life. My brothers, Abdurrahman and Ahmed, also supported me and helped me during my studies. I would not have done any of this without their support.

Finally, I would like to thank the School of Computer Science, the faculty, the staff and my friends here. I am grateful for the friendships that I was able to develop, and the support from everyone whom I became friends with at the School. Together, they made it a pleasant and enjoyable atmosphere. 


\section{Contents}

1 Introduction 1

1.1 Introduction . . . . . . . . . . . . . . . . . . . 1

1.2 Motivation for the Thesis . . . . . . . . . . . . . . . 3

1.3 Objectives of the Thesis $\ldots \ldots \ldots \ldots \ldots$

1.4 Problem Statement . . . . . . . . . . . . . . . . . . . 4

1.5 Contributions of the Thesis $\ldots \ldots \ldots \ldots \ldots$

1.6 Hardware/Software Used for the Simulations . . . . . . . . . . . 6

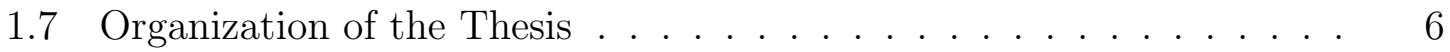

2 Survey of the Relevant Fields $\quad 8$

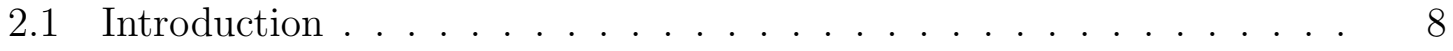

2.2 The Learning Automata $(\mathrm{LA}) \ldots \ldots \ldots \ldots$

2.2 .1 What is Learning? . . . . . . . . . . . . . . . . 9

2.2 .2 Learning Automata (LA) . . . . . . . . . . . . . . 9

2.2 .3 Environment . . . . . . . . . . . . . . . . . . . 11

2.2 .4 Automaton . . . . . . . . . . . . . . . . 11

2.2.5 Structures and Classes of LA . . . . . . . . . . . 14

2.2.6 Fixed Structure Stochastic Learning Automata (FSSA) . . . . 14

2.2.7 Variable Structure Stochastic Learning Automata (VSSA) . . 19

2.2.8 Estimator and Pursuit Algorithms . . . . . . . . . . . . . . 23

2.2 .9 LA for Non-Stationary REs . . . . . . . . . . . . . . . 23

2.2 .10 Conclusions on the Review of LA . . . . . . . . . . . 24

2.3 Elevator-like Problems . . . . . . . . . . . . . . . . . . . . . 25 
2.3 .1 Introduction . . . . . . . . . . . . . . . . . 25

2.3.2 Single Elevator Problem _ . . . . . . . . . . . . 26

2.3 .3 Multiple Elevator Problem . . . . . . . . . . . . . . . . . 29

2.3.4 Elevator-like Problems . . . . . . . . . . . . . . . . 34

2.4 Chapter Conclusion . . . . . . . . . . . . . . . . . . . 35

3 Solutions for the SEP $\quad 36$

3.1 Introduction . . . . . . . . . . . . . . . . 36

3.2 Models of Computation . . . . . . . . . . . . . . . . . . . 37

3.3 Simulation Settings . . . . . . . . . . . . . . . . . . . 38

3.4 Previous Solutions . . . . . . . . . . . . . . . . . . . . . . . 39

3.4.1 Do_Nothing Policy: SEP1 . . . . . . . . . . . . . . 43

3.4 .2 Myopic Policy: SEP2 . . . . . . . . . . . . . . . . 46

3.5 LA-Based Solutions . . . . . . . . . . . . . . . . . . . 52

3.5 .1 Problem Modelling . . . . . . . . . . . . . . . 52

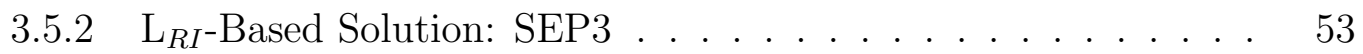

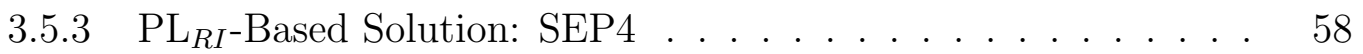

3.6 Discussion . . . . . . . . . . . . . . . . . . . . . . . . . . . . 62

3.7 Conclusion . . . . . . . . . . . . . . . . . . . . 65

4 Solutions for the MEP $\quad 67$

4.1 Introduction . . . . . . . . . . . . . . . . . 67

4.2 Models of Computation . . . . . . . . . . . . . . . . 68

4.3 Simulation Settings . . . . . . . . . . . . . . . . . . 68

4.4 Previous Solutions . . . . . . . . . . . . . . . . . . . 69

4.4.1 Do Nothing Policy: MEP1 _ . . . . . . . . . . . . . 70

4.4 .2 Myopic Policy: MEP2 _. . . . . . . . . . . . . . . . 72

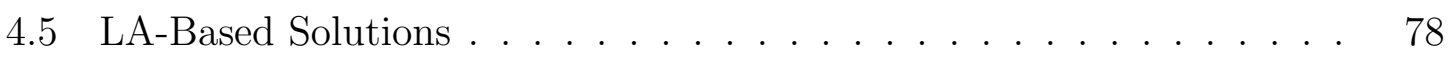

4.5 .1 Problem Modelling . . . . . . . . . . . . . . . . . . 79

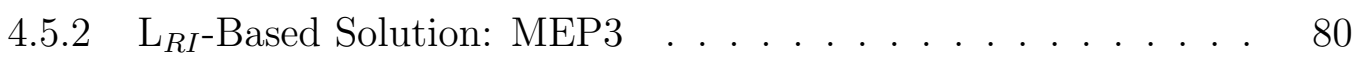

4.5.3 $\mathrm{PL}_{R I}$-Based Solution: MEP4 . . . . . . . . . . . . 84

4.6 Discussion . . . . . . . . . . . . . . . . . . . . . . . . . . . . . 90 
4.7 Conclusion . . . . . . . . . . . . . . . . . . . . . . . . . . . 92

5 Summary, Conclusions and Future Work $\quad 94$

5.1 Summary and Conclusion . . . . . . . . . . . . . 94

5.2 Conclusion and Future Work . . . . . . . . . . . . . . . . . 96

$\begin{array}{ll}\text { Appendices } & 98\end{array}$

$\begin{array}{ll}\text { A SEP Results } & 99\end{array}$

$\begin{array}{ll}\text { B MEP Results } & 107\end{array}$

$\begin{array}{ll}\text { Bibliography } & 115\end{array}$ 


\section{List of Figures}

2.1 The Automaton-Environment Feedback Loop. . . . . . . . . . . . . 10

2.2 The state transition graphs for Tsetlin's $L_{2 N, 2} \ldots \ldots \ldots \ldots \ldots \ldots$

2.3 The state transition graphs for Krinsky's $K r_{2 N, 2} \ldots \ldots \ldots \ldots \ldots$

2.4 The state transition graphs for Krylov's $L_{2 N, 2} \ldots \ldots \ldots \ldots \ldots \ldots$

3.1 A typical bimodal distribution for a 12-floor building and the corresponding discretized probabilities constituting $\mathcal{C} \ldots \ldots \ldots$

3.2 The Average Waiting Time for the "Do Nothing" Policy, SEP1, for an ensemble of 200 experiments for the case of the Exp distribution. . . 47

3.3 Average Waiting Time for the "Myopic Policy", SEP2, for an ensemble of 200 experiments for the case of Experiment $E x(1) \ldots \ldots \ldots$

3.4 The Average Waiting Time for SEP1 vs. SEP2, for an ensemble of 200 experiments for the case of the Exp distribution. . . . . . . . . . 52

3.5 The Average Waiting Time for the $L_{R I}$ solution, SEP3, for an ensemble of 200 experiments for the case of the Exp distribution. . . . . . . 58

3.6 The Average Waiting Time for the $P L_{R I}$ solution, SEP4, for an ensemble of 200 experiments for the case of the Exp distribution. . . . .

3.7 The Average Waiting Time for the $L_{R I}$ solution, SEP3, vs. $P L_{R I}$ solution, SEP4, for an ensemble of 200 experiments for the case of the Exp distribution. . . . . . . . . . . . . . .

3.8 The Average Waiting Time for SEP1, SEP2, SEP3 and SEP4 for an ensemble of 200 experiments for the case of the Exp distribution, given in the graph as $P 1, P 2, L_{R I}, P L_{R I}$ respectively. . . . . . . 
4.1 The Average Waiting Time for the "Do Nothing" Policy, MEP1, for an ensemble of 200 experiments for the case of the Exp distribution. . 73

4.2 The Average Waiting Time for the Myopic Policy, MEP2, for an ensemble of 200 experiments for the case of the Exp distribution. . . . . 78

4.3 The Average Waiting Time for the MEP1 vs. MEP2, for an ensemble of 200 experiments for the case of the Exp distribution. . . . . . . . . 79

4.4 The Average Waiting Time for the $L_{R I}$ solution, MEP3, for an ensemble of 200 experiments for the case of the Exp distribution. . . . . . .

4.5 The Average Waiting Time for the $P L_{R I}$ solution, MEP4, for an ensemble of 200 experiments for the case of the Exp distribution. . . . .

4.6 The Average Waiting Time for the MEP3 vs. MEP4, for an ensemble of 200 experiments for the case of the Exp distribution. . . . . . . . . 90

4.7 The Average Waiting Time for MEP1, MEP2, MEP3 and MEP4 for an ensemble of 200 experiments for the case of the Exp distribution. .

A.1 The Average Waiting Time for SEP1, SEP2, SEP3 and SEP4 for an ensemble of 200 experiments for the case of the Exp distribution for an 8-floor setting. . . . . . . . . . . . . . . . . . 100

A.2 The Average Waiting Time for SEP1, SEP2, SEP3 and SEP4 for an ensemble of 200 experiments for the case of the InvExp distribution for an 8-floor setting. . . . . . . . . . . . . . . . . .

A.3 The Average Waiting Time for SEP1, SEP2, SEP3 and SEP4 for an ensemble of 200 experiments for the case of the Gaussian distribution for an 8-floor setting. . . . . . . . . . . . . . . . . . . 101

A.4 The Average Waiting Time for SEP1, SEP2, SEP3 and SEP4 for an ensemble of 200 experiments for the case of the Bimodal distribution for an 8-floor setting. . . . . . . . . . . . . . . . . . . 101

A.5 The Average Waiting Time for SEP1, SEP2, SEP3 and SEP4 for an ensemble of 200 experiments for the case of the Exp distribution for an 16-floor setting. . . . . . . . . . . . . . . . 102 
A.6 The Average Waiting Time for SEP1, SEP2, SEP3 and SEP4 for an ensemble of 200 experiments for the case of the InvExp distribution for an 16-floor setting. . . . . . . . . . . . . . . . . . . 103

A.7 The Average Waiting Time for SEP1, SEP2, SEP3 and SEP4 for an ensemble of 200 experiments for the case of the Gaussian distribution for an 16 -floor setting. . . . . . . . . . . . . . . . . . 103

A.8 The Average Waiting Time for SEP1, SEP2, SEP3 and SEP4 for an ensemble of 200 experiments for the case of the Bimodal distribution for an 16-floor setting. . . . . . . . . . . . . . . . . . . . 104

A.9 The Average Waiting Time for SEP1, SEP2, SEP3 and SEP4 for an ensemble of 200 experiments for the case of the Exp distribution for an 20 -floor setting. . . . . . . . . . . . . . . . 105

A.10 The Average Waiting Time for SEP1, SEP2, SEP3 and SEP4 for an ensemble of 200 experiments for the case of the InvExp distribution for an 20 -floor setting. . . . . . . . . . . . . . . . . 105

A.11 The Average Waiting Time for SEP1, SEP2, SEP3 and SEP4 for an ensemble of 200 experiments for the case of the Gaussian distribution for an 20-floor setting. . . . . . . . . . . . . . . . 106

A.12 The Average Waiting Time for SEP1, SEP2, SEP3 and SEP4 for an ensemble of 200 experiments for the case of the Bimodal distribution for an 20 -floor setting. . . . . . . . . . . . . . . . . 106

B.1 The Average Waiting Time for MEP1, MEP2, MEP3 and MEP4 for an ensemble of 200 experiments for the case of the Exp distribution for an 8 -floor setting. . . . . . . . . . . . . . . . . . 108

B.2 The Average Waiting Time for MEP1, MEP2, MEP3 and MEP4 for an ensemble of 200 experiments for the case of the InvExp distribution for an 8-floor setting. The graph for MEP3 showed a superior performance to MEP4, and this was probably a statistical anomaly. . . . . . . 108 
B.3 The Average Waiting Time for MEP1, MEP2, MEP3 and MEP4 for an ensemble of 200 experiments for the case of the Gaussian distribution for an 8-floor setting. . . . . . . . . . . . . . . . . 109

B.4 The Average Waiting Time for MEP1, MEP2, MEP3 and MEP4 for an ensemble of 200 experiments for the case of the Bimodal distribution for an 8-floor setting. . . . . . . . . . . . . . . . . . . 109

B.5 The Average Waiting Time for MEP1, MEP2, MEP3 and MEP4 for an ensemble of 200 experiments for the case of the Exp distribution for an 16-floor setting. . . . . . . . . . . . . . . . . . . 110

B.6 The Average Waiting Time for MEP1, MEP2, MEP3 and MEP4 for an ensemble of 200 experiments for the case of the InvExp distribution for an 16-floor setting. . . . . . . . . . . . . . . . . . 111

B.7 The Average Waiting Time for MEP1, MEP2, MEP3 and MEP4 for an ensemble of 200 experiments for the case of the Gaussian distribution for an 16-floor setting. . . . . . . . . . . . . . . . . . 111

B.8 The Average Waiting Time for MEP1, MEP2, MEP3 and MEP4 for an ensemble of 200 experiments for the case of the Bimodal distribution for an 16-floor setting. . . . . . . . . . . . . . . . . . . 112

B.9 The Average Waiting Time for MEP1, MEP2, MEP3 and MEP4 for an ensemble of 200 experiments for the case of the Exp distribution for an 20-floor setting. . . . . . . . . . . . . . . . . . .

B.10 The Average Waiting Time for MEP1, MEP2, MEP3 and MEP4 for an ensemble of 200 experiments for the case of the InvExp distribution for an 20-floor setting. . . . . . . . . . . . . . . . . . .

B.11 The Average Waiting Time for MEP1, MEP2, MEP3 and MEP4 for an ensemble of 200 experiments for the case of the Gaussian distribution for an 20-floor setting. . . . . . . . . . . . . . . . . . . . 113

B.12 The Average Waiting Time for MEP1, MEP2, MEP3 and MEP4 for an ensemble of 200 experiments for the case of the Bimodal distribution for an 20-floor setting. . . . . . . . . . . . . . . . . . . . 114 


\section{Chapter 1}

\section{Introduction}

\section{$1.1 \quad$ Introduction}

Right from its infancy, the goal of the field of Artificial Intelligence (AI) has been to make computers respond intelligently in everyday and challenging situations. Turing, in his Turing test, suggested that a machine could be considered to possess AI if a human observer would not have been able to distinguish its behaviour from the behaviour of a real human being. The goal, although it has been lofty, has been achieved to a phenomenal degree. AI-based computer programs have challenged and beaten the best players in many games, including Chess and Go.

It is staggering to record the areas in which AI has been used. Machine learning, pattern recognition, medical diagnosis and voice-operated systems are commonplace in today's world. AI has been used in practically every single application domain.

This thesis considers a field in which AI has not been applied much. Consider a tennis player who is moving within his side of the court. After he hits the ball, the question that he encounters is to know where he should place himself so as to best counter his opponent's response. This can be modelled as a parking problem, i.e., where should the player "park" himself so that when his opponent hits the ball, it is in the vicinity of where he has parked. We could refer to problems of this type as "elevator-like" problems. They are, in fact, in a unidimensional domain, related to the problem of where an elevator within a building should be parked. In other words, 
if there is a building with $n$ floors and if the elevator car is requested at floor $i$, where should the car move to after the passenger has been dropped off? Of course, the answer to this question depends on the criterion function, but it is expedient to work with the understanding that the car should be parked near to the next passenger call. The same analogy can be seen if we extend the problem domain to know where police or emergency vehicles should be parked so as to be available, in the shortest possible time, for the next call.

Although the above problems are, in general, transportation problems, their common facet can also be extended to other domains. For example, one could consider the problem of where the read/write disk head in a memory bank should be placed so that it can access data more expeditiously. Similarly, one could consider the problem of where security guards, who move on a rotational basis, should be placed so that the facility is maximally secured. Indeed, our problem model can be extended to consider improving underwater communication systems by determining where the underwater sensors should be located.

In this thesis, we refer to all of these problems as "Elevator-like" problems, and this is the primary focus of the research. However, to render the problem to be nontrivial, we assume that "the world" in which we are operating is stochastic and that the underlying distributions are unknown. For example, in the case of the tennis player, we assume that there is a distribution for the place where the opponent will place his counter-shot, but that this distribution is unknown.

Our aim is to model all these problems in a unidimensional space by means of incorporating them into an elevator "setting". Such an elevator system is characterized by two distributions $\mathcal{C}=\left\{c_{1}, \ldots, c_{n}\right\}$ and $\mathcal{D}=\left\{d_{1}, \ldots, d_{n}\right\}$, where $c_{i}$ is the probability of receiving a single-passenger call from floor $i$, and $d_{j}$ is the probability of the "elevator" being asked to move to destination $j$. The question now is that of determining where the "elevator" should be "parked" so that it is ready for the next call.

Thus far, we have discussed the scenario when there is only a single "elevator" car. The problem becomes far more complex when we are dealing with multiple "elevators". In such a case, we have to consider how all the "elevators" can be moved around so that one of them is close enough when the next request is made. 
The goal of this thesis is to provide solutions to general elevator-like problems using a subfield of AI referred to as Learning Automata (LA).

\subsection{Motivation for the Thesis}

Elevator-like problems have been tackled before by researchers. However, the focus has been on other perspectives and not on parking considerations. Previous research sought for solutions to these problems, but was usually concerned with optimizing a host of metrics when it concerns the elevator's efficiency. Researchers tried to minimize the energy consumption, the total travel time or the scheduling of the cars, and few have tackled the problem of determining the optimal parking position for the elevator during its idle period.

From an alternate perspective, researchers have used various methods and tools to achieve the optimization of the above-mentioned metrics. For example, they have used Artificial Neural Networks (ANNs), Ant Colony Optimization or linear programming to solve the elevator scheduling problem. Our investigations show that none of the research reported in the literature has used the field of LA to tackle the elevator problem.

This is the foundation for the motivation of our thesis. Our goal is to tackle the problem of parking single and multiple elevators during their idle period by incorporating LA-based solutions. The primary purpose of the exercise will be to minimize the waiting time for the passengers.

\subsection{Objectives of the Thesis}

The main objective of this thesis is to investigate how LA can be used to solve reallife elevator problems, and by extension, elevator-like problems. To achieve this, we have sub-divided our objective into smaller ones, which can, collectively, represent the overall objective.

First of all, we would like to see which competitive models can be used as benchmarks against the LA-based solutions that we intend to propose. In this regard, we 
will introduce two competitive solutions. The first one is the dominant policy in modern-day buildings. It depends solely on the destination floor, and the controller decides to park the car at that floor after dropping the passenger off. The second solution, is the optimal one, and is based on knowing the calling distribution a priori. It involves exhaustively searching through the available options to find the best (optimal) parking floor. While the first solution serves as an upper bound for the solution to the problem considered here, the second solution provides a lower bound.

Secondly, we would like to see how the elevator can be modelled in such a way that makes it applicable to be solved using LA-based schemes. We will thus divide the fundamental problem into two main scenarios, namely, the Single Elevator Problem (SEP) and the Multi-Elevator Problem (MEP). Our objective is to provide two new LA-based solutions for each setting so as to get as close as possible to the optimal solutions.

\subsection{Problem Statement}

The problem we are trying to tackle can be stated as follows: We have a specific building with $n$ floors and $e$ elevators. The floors and passengers are characterized by distributions $\mathcal{C}=\left\{c_{1}, \ldots, c_{n}\right\}$ and $\mathcal{D}=\left\{d_{1}, \ldots, d_{n}\right\}$, where $c_{i}$ is the probability of receiving a call from floor $i$, and $d_{j}$ is the probability that the elevator drops the passenger off at floor $j$. These distributions are unknown to the decision-making algorithm, and our goal is to design LA-based solutions such that they adaptively determine a set of floors for the $e$ elevators to park at during the idle period so as to minimize the passengers' waiting time.

The metric used as a performance measure in our study is the Average Waiting Time (AWT) of the passengers. This is clearly a function of the number of floors, and of also how close the converged solution is to the optimal floor. 


\subsection{Contributions of the Thesis}

In the context of what we have discussed above, we list the contributions of this thesis as follows:

- We have surveyed a subfield of AI, namely the field of Learning Automata (LA), and have concluded that it has not been used previously to solve the elevator problem.

- We were able to identify two different models of computations for the elevator problem, where the first requires the calling distribution to be known a priori, and the second does not need such information.

- We were able to model the elevator problem in such a way that it can be solved using LA approaches.

- We introduced a Linear Reward-Inaction $\left(L_{R I}\right)$-based solution to tackle the single elevator problem. It has been referred to as SEP3.

- We also presented an improvement on SEP3 by including the so-called pursuit phenomenon into the LA solution. This led to the $P L_{R I}$-based solution, referred to as SEP4, and this yielded better results and faster convergence than SEP3.

- We also tackled the multi-elevator problem, which is the more complex scenario. Here too we presented an $L_{R I}$-based solution, referred to as MEP3. This resulted in an increase of up to $90 \%$ in the performance of the elevators.

- We proceeded to present the enhancement to the MEP3 by again including the so-called pursuit concept. This yielded the second and superior $P L_{R I}$-based solution, MEP4.

- To summarize, we have shown that LA-based solutions can solve elevatorlike problems without requiring any knowledge of the underlying distributions. Amazingly enough, the results and solutions that they yielded are near-optimal. 


\subsection{Hardware/Software Used for the Simulations}

Before we move into the body of the thesis, in the interest of completeness, we state the details of the platforms on which the simulations in this thesis were done:

- The hardware used for the simulations in the thesis was an Intel Core i7-6800K CPU with $16 \mathrm{GBs}$ of physical memory installed;

- The language used for the simulations in the thesis was 'Python' with a version 3.6 and using the software 'PyCharm' version 2018.1.

- It is pertinent to mention that there are two fundamental methods to evaluate simulations. The first computes the average of the results obtained and also the standard deviations within a pre-specified margin of error. The alternative method is the one used in the field of LA, and it involves computing the ensemble average of the time average of a large number of experiments. This is the method that we follow in this thesis.

\subsection{Organization of the Thesis}

Chapter 2 presents an overview of the two relevant fields that pertain to this thesis. The chapter first surveys the field of LA. It mentions how it has been central to reinforcement learning, and the ways by which it has been applied to an ensemble of problem domains. Thereafter, we survey Elevator-like Problems (ELPs) briefly stated above. The chapter includes a survey of how previous researchers have tackled the problem from different perspectives.

Chapter 3 discusses the case of single elevator buildings. It presents two previous solutions that we have considered as benchmarks in the thesis. The first solution, referred to as SEP1, provides an upper bound for the Average Waiting Time (AWT), and SEP2, the second solution, provides us with a lower bound for the AWT. This chapter also includes our new LA-based solutions for the SEP, namely SEP3, an $L_{R I^{-}}$ based solution, and SEP4, a $P L_{R I}$-based solution. The chapter includes a comparative study of the four schemes. 
Chapter 4 presents the case of the multi-elevator building settings. Again, the chapter presents the solutions that we have used as benchmarks, which are MEP1 that extends SEP1, and MEP2 that extends SEP2 for the multi-elevator domain. The chapter then submits our extended LA-based solutions for the MEP, which we refer to as MEP3 and MEP4. We also include results that show how they performed, and compare them to the benchmark solutions.

Chapter 5 concludes the thesis. It presents a summary of each chapter and discusses potential future work, namely, solving the ELP in non-stationary Environments, and the task of combining different dispatching policies in such settings.

The results presented in the bodies of Chapters 3 and 4 are representative of the entire set of results that we have obtained. In the interest of readability, the complete suite of results are presented in Appendix A and Appendix B respectively. 


\section{Chapter 2}

\section{Survey of the Relevant Fields}

\section{$2.1 \quad$ Introduction}

In this chapter, we will present a survey of the two relevant fields that pertain to this thesis. First of all, we will survey the field of Learning Automata (LA). We will mention how it has been central to reinforcement learning, and the algorithms by which it has been applied to an ensemble of problem domains.

Secondly, we will discuss Elevator-like Problems (ELPs) that were introduced in Chapter 1. We will also survey how previous researchers tackled the problem from different perspectives. From a "controls" perspective we shall see how they have tried to optimize the elevators' control systems to reduce, for example, the energy consumption, waiting time, travel time and/or other metrics. The methods that have been used, and the corresponding results obtained, will be briefly presented.

Our goal in the following chapters will be to see how we can obtain LA-based solutions to ELPs, in order to optimize the waiting time and other metrics.

\subsection{The Learning Automata (LA)}

In this section, we will survey the field of LA. First, we will introduce some concepts of learning theory and the phenomenon of learning. Next, we are going to define LA, give a mathematical definition to it and talk about how it is used. After that, we 
will present the different types and classes of LA and the differences between them, before concluding this section.

\subsubsection{What is Learning?}

In this thesis, our work is based on a paradigm that involves "Learning Automata" (LA). To understand what LA are, we are first going to clarify what "Learning" itself means. By quantifying the behaviour associated with the process of learning, we shall create a model that can be used to formulate this process.

The definition of "learning" traces back to psychologists who studied the concepts associated with it. They investigated the behaviour of an intelligent individual/being when it interacted with an environment (or with an agent) that could provide it with some kind of feedback, using which it could update its behaviour. By the process of repeating this cycle, scientists were able to notice an increase in the ability of the individual/being to react in a better way and/or to take an action that would not be detrimental. One of the main areas of research in Computer Science involves Reinforcement Learning, and this field is precisely based on such a model of learning. It also constitutes the foundations for some of the most fundamental tools in Machine Learning (ML) and Artificial Neural Networks (ANN), essentially those that characterize Learning, and in making decisions so as to minimize the penalties or maximize the rewards that the intelligent machine receives.

\subsubsection{Learning Automata (LA)}

We now concentrate on the field that we shall work in, namely, that of LA. The concept of LA was first introduced in the early 1960's in the pioneering research done by Tsetlin [37]. He proposed a computational learning scheme that can be used to learn from a random (or stochastic) Environment which offers a set of actions for the automaton to choose from. The automaton's goal is to pick the best action that maximizes the reward received from the Environment and minimizes the penalty. The evaluation is based on a function that permits the Environment to stochastically measure how good an action is, and to thereafter send an appropriate feedback signal 
to the automata.

After the introduction of LA, different structures of LA, such as the deterministic and the stochastic schemes, were introduced and studied by the famous researchers Tsetlin, Krinsky and Krylov in [37] and Varshavskii in [38].

The field of LA, like many of the Reinforcement Learning techniques, has been used in a variety of problems, mainly optimization problems, and in many fields of AI. It has been used in neural network adaptation [11], solving communication and networking problems [12], [18], [20] and [21], in distributed scheduling problems [25], and in the training of Hidden Markov Models [7].

In this survey, we will cover the relevant background required to help the reader understand the fundamental concepts for our proposed work ${ }^{1}$.

In Figure 2.1, we have the general stochastic learning model associated with LA. The components of the model are the Random Environment, the Automaton, the Feedback received from the environment and the Actions chosen by the automaton.

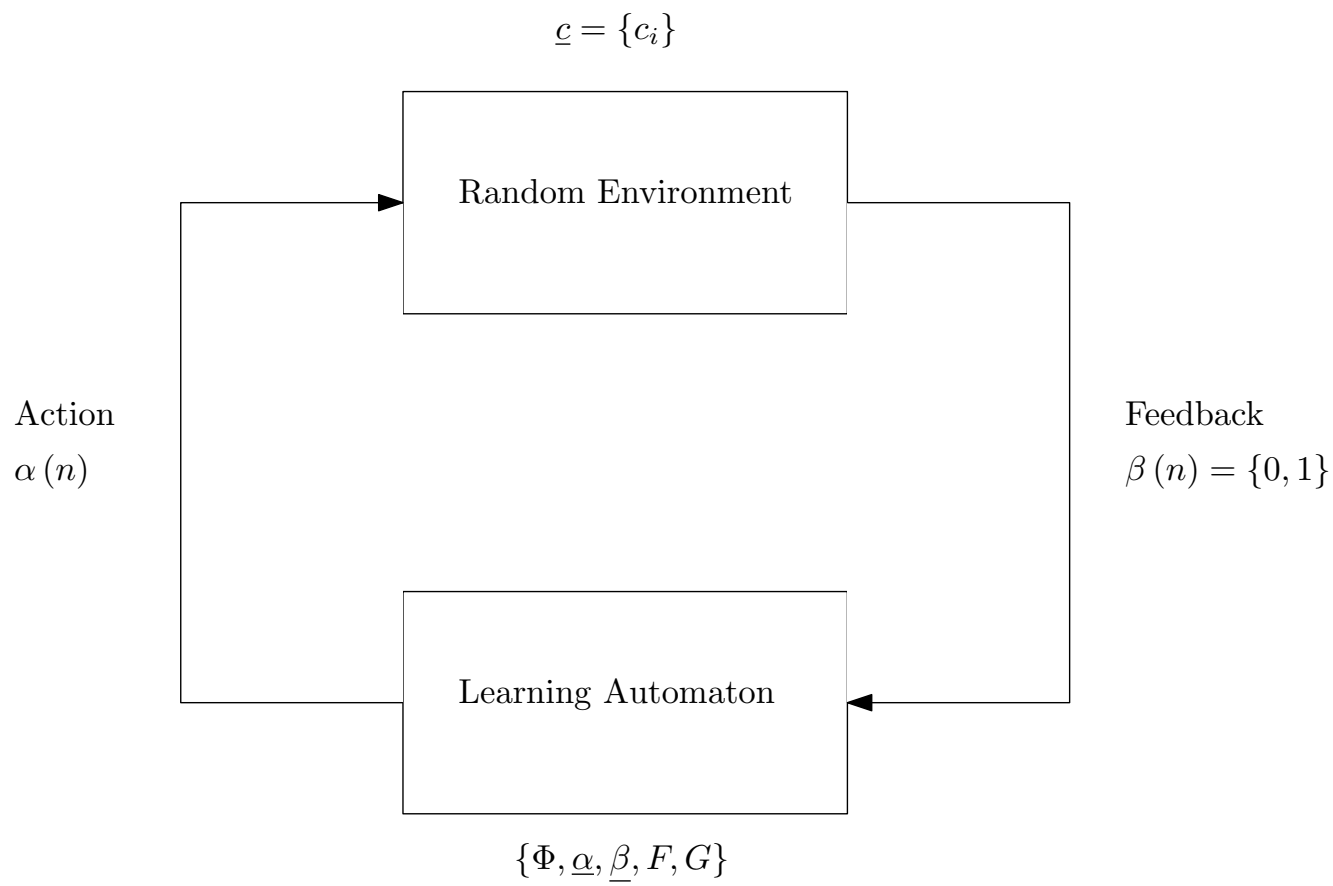

Figure 2.1: The Automaton-Environment Feedback Loop.

\footnotetext{
${ }^{1}$ We will not go through irrelevant details and/or the proofs of the LA-related claims.
} 


\subsubsection{Environment}

We first define the stochastic Random Environment that the automaton interacts with. Initially, the automaton picks an action from the set of actions available to it and communicates it to the environment. The Environment evaluates that action according to a random function, and sends back to the automaton a feedback signal, depending on whether that action resulted in a reward or a penalty.

The Environment can be mathematically described as a triple:

$$
\mathbb{E}=\{\underline{\alpha}, \underline{c}, \underline{\beta}\}
$$

where:

- $\underline{\alpha}$ : is the set of actions $\left\{\alpha_{1}, \alpha_{2}, \ldots, \alpha_{r}\right\}$

- $\underline{\beta}$ : is the set feedbacks, where, typically, $\underline{\beta}=\{0,1\}$, and is transmitted from $\mathbb{E}$ to the LA

- $\underline{c}$ : is the set of penalty probabilities associated with the Environment, and it corresponds to the set of actions $\underline{\alpha}$ where:

$$
c_{i}=\operatorname{Pr}\left[\beta(n)=0 \mid \alpha(n)=\alpha_{i}\right] \quad(i=1,2, \ldots, r) .
$$

The Environment, $\mathbb{E}$, can be classified as being one of two types. The first one is a stationary Environment, and this is when the penalty probabilities are constant. On the other hand, a non-stationary Environment is characterized by the fact that the penalty probabilities change with time.

\subsubsection{Automaton}

Narendra and Thathachar [15], the early pioneers of the field of LA, define the LA as a quintuple:

$$
\{\Phi, \underline{\alpha}, \underline{\beta}, F(\cdot, \cdot), G(\cdot)\}
$$

where: 
- $\Phi$ : represents the set of states. $\phi(n)$ is the current state at time $n$. Hence, we can formally describe: $\Phi=\left\{\phi_{1}, \phi_{2}, \ldots, \phi_{r}\right\}$.

- $\underline{\alpha}$ : represents the set of actions that the automaton can pick from. $\alpha(n)$ is the action selected by the automaton at time $n$. Thus, $\underline{\alpha}=\left\{\alpha_{1}, \alpha_{2}, \ldots, \alpha_{r}\right\}$.

- $\beta$ : represents the set of possible feedback signals transmitted by the Environment, where $\beta=\{0,1\} . \beta=0$ is the case when the Environment rewards the taken action. On the other hand, $\beta=1$ is the case when the environment penalizes the taken action.

- $F$ : represents the transition function for the LA from the current state $\phi(n)$ to the next state $\phi(n+1)$. Formally, $\phi(n+1)=F(\phi(n), \beta(n))$

- $G$ : represents the output function of the automaton, where $G$ can be stochastic or deterministic. This output specifies the selection of the action by the LA. Formally, $\alpha(n)=G(\phi(n))$.

The LA achieves its learning process as an iterative operation that is based on the Environment and the interaction between them. This process consists of two main steps which are, policy evaluation, which is how the Environment evaluates the selected action. Moreover, the second step involves policy improvement, where the LA improves the probability of selecting an action that would maximize the reward received from the Environment.

Hence, we can formalize the LA by having the following equation $\forall n$ :

$$
\begin{aligned}
& p_{i}(n)=\operatorname{Pr}\left[\alpha(n)=\alpha_{i}\right]:(i=1, \ldots, r), \text { and } \\
& \sum_{i=1}^{r} p_{i}(n)=1,
\end{aligned}
$$

where $p_{i}(n)$ is the probability of the LA choosing an action $\alpha_{i}$ at time $n$, and the collective probability vector has all the action probabilities in the corresponding indices of that vector, $P(n)$.

One quantity that is used to measure the performance of the LA is the average penalty, $M(n)$, for the probability vector $P(n)$. This average penalty is specified with 
the following equation:

$$
\begin{aligned}
M(n) & =E[\beta(n) \mid P(n)]=\operatorname{Pr}[\beta(n)=1 \mid P(n)] \\
& =\sum_{i=1}^{r} c_{i} p_{i}(n) .
\end{aligned}
$$

Consider the pure-chance automaton, which is when we do not have any preference or biases for any actions. In this automaton, the average penalty $M_{0}$ is given:

$$
M_{0}=\frac{1}{r} \sum_{i=1}^{r} c_{i} .
$$

This pure-chance automaton is used to compare the performances of different LA schemes. For the LA to be a well-performing LA, it has to be at least better than the pure-chance LA. Hence, we compare $E[M(n)]$ with $M_{0}$ and determine whether the expected average penalty is better than the pure-chance machine or not.

We can characterize the LA by four possibilities having four different cases for $E[M(n)]$. If the LA performs better than the pure-chance machine, we say that we have an Expedient LA, where:

$$
\lim _{n \rightarrow \infty} E[M(n)]<M_{0} .
$$

Secondly, we may have an optimal LA when:

$$
\lim _{n \rightarrow \infty} E[M(n)]=c_{l}, \quad \text { where } \quad c_{l}=\min _{i}\left\{c_{i}\right\} .
$$

Thirdly, an LA is said to be $\epsilon$-optimal whenever:

$$
\lim _{n \rightarrow \infty} E[M(n)] \leq c_{l}+\epsilon,
$$

where $\epsilon>0$ is an arbitrarily small user-defined value.

Finally, we say that the LA is Absolutely Expedient when:

$$
E[M(n+1) \mid P(n)]<M(n) .
$$




\subsubsection{Structures and Classes of LA}

In this section, we are going to discuss the different structures and classes of LA.

As we saw before, LA have two main functions that it uses, the transition function, $F(\cdot, \cdot)$, and the output function, $G(\cdot)$. These two functions are what determine the type of the LA. When both of these functions are deterministic, the LA is classified as being a deterministic LA. On the other hand, when at least one of them is stochastic, we have a stochastic LA. The difference is that in the deterministic case, we can precisely determine the next state and action for the LA from only a knowledge of its current state. However, in stochastic version, we have only a transition probability that is used to determine the next state stochastically. This transition probability is defined by the matrix $F$ where:

$$
f_{i j}^{(\beta)}=\operatorname{Pr}\left[\phi(n+1)=\phi_{j} \mid \phi(n)=\phi_{i}, \beta(n)=\beta\right]: i, j=1,2, \cdots, r .
$$

As one sees, each entry corresponds to the transition probability of the LA going from the current state to the next state.

LA can be classified into two main classes, namely:

1. Fixed Structure Stochastic Automata (FSSA), and

2. Variable Structure Stochastic Automata (VSSA)

Narendra and Thathachar, in [15] explained, in detail, the various structures and classes of LA. We summarize these concepts below.

\subsubsection{Fixed Structure Stochastic Learning Automata (FSSA)}

In this section, we discuss the first family of LA, namely FSSA. In FSSA, the transition probabilities are constant and do not change with time. To describe and analyze any FSSA, one uses the theory of Markov Chains. In particular, we present the three main machines due to Tsetlin, Krinsky and Krylov. All these FSSA use the theory of Markov Chains to analyze the quality of their performances. 


\section{The Two Action Automaton with Memory: $L_{2 N, 2}$}

In early 1960's [37], the first LA scheme was introduced by Tsetlin, and it is referred to as $L_{2 N, 2}$. The $L_{2 N, 2}$ automaton has $2 N$ states $\left\{\phi_{1}, \ldots, \phi_{2 N}\right\}$ and two actions ${ }^{2}$ $\left\{\alpha_{1}, \alpha_{2}\right\}$. The $2 N$ states for $L_{2 N, 2}$ scheme are divided into two subsets. The first set of states, $\left\{\phi_{1}, \ldots, \phi_{N}\right\}$, corresponds to the first action, $\alpha_{1}$, and the rest of states, i.e., $\left\{\phi_{N+1} \ldots, \phi_{2 N}\right\}$, correspond to the second action, $\alpha_{2}$.

In the $L_{2 N, 2}$ scheme described in Figure 2.2, we can see how the state transitions are defined when the automaton receives a reward (i.e., $\beta=0$ ) or a penalty (i.e., $\beta=1)$. When the machine receives a reward, the LA goes one step deeper in the memory of that selected action that yielded the reward. On the other hand, the LA moves one step towards the states representing the other action when it receives a penalty.

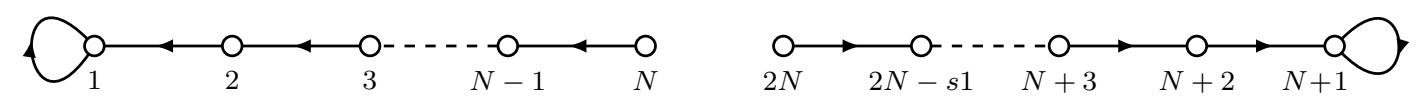

On a reward, $\beta=0$

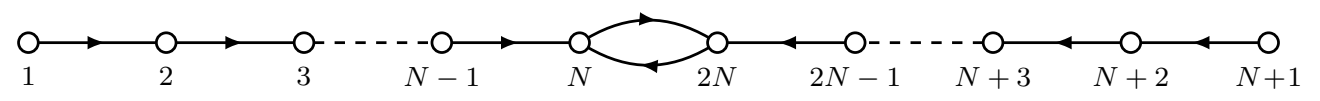

On a penalty, $\beta=1$

Figure 2.2: The state transition graphs for Tsetlin's $L_{2 N, 2}$.

\footnotetext{
${ }^{2}$ This can be easily be generalized for the case when there $r$ actions.
} 
The transition matrices for Tsetlin, $F^{(0)}$ and $F^{(1)}$, are defined in Eq. (2.9) and Eq. (2.10) where $F^{(0)}$ is the transition matrix when the automaton receives a reward and $F^{(1)}$ is the matrix when it receives a penalty with:

$$
\begin{aligned}
F_{(2 N \times 2 N)}^{(0)} & =\left[\begin{array}{ccccc|ccccc}
1 & 0 & 0 & \cdot & \cdot & \cdot & \cdot & \cdot & \cdot & \cdot \\
1 & 0 & 0 & \cdot & \cdot & \cdot & \cdot & \cdot & \cdot & \cdot \\
0 & 1 & 0 & \cdot & \cdot & \cdot & \cdot & \cdot & \cdot & \cdot \\
\cdot & \cdot & \cdot & \cdot & \cdot & \cdot & \cdot & \cdot & \cdot & \cdot \\
0 & 0 & 0 & 1 & 0 & 0 & 0 & \cdot & \cdot & \cdot \\
\hline \cdot & \cdot & \cdot & \cdot & \cdot & 1 & 0 & 0 & \cdot & \cdot \\
\cdot & \cdot & \cdot & \cdot & \cdot & 1 & 0 & 0 & \cdot & \cdot \\
\cdot & \cdot & \cdot & \cdot & \cdot & 0 & 1 & 0 & \cdot & \cdot \\
\cdot & \cdot & \cdot & \cdot & \cdot & \cdot & \cdot & \cdot & \cdot & \cdot \\
\cdot & \cdot & \cdot & \cdot & \cdot & 0 & \cdot & \cdot & 1 & 0
\end{array}\right], \\
F_{N \times 2 N)}^{(1)} & =\left[\begin{array}{ccccc|ccccc}
0 & 1 & 0 & \cdot & \cdot & \cdot & \cdot & \cdot & \cdot & \cdot \\
0 & 0 & 1 & \cdot & \cdot & \cdot & \cdot & \cdot & \cdot & \cdot \\
\cdot & \cdot & \cdot & \cdot & \cdot & \cdot & \cdot & \cdot & \cdot & \cdot \\
0 & 0 & 0 & \cdot & 1 & \cdot & \cdot & \cdot & \cdot & \cdot \\
0 & 0 & 0 & \cdot & 0 & \cdot & \cdot & \cdot & \cdot & 1 \\
\hline \cdot & \cdot & \cdot & \cdot & \cdot & 0 & 1 & 0 & \cdot & \cdot \\
\cdot & \cdot & \cdot & \cdot & \cdot & 0 & 0 & 1 & \cdot & \cdot \\
\cdot & \cdot & \cdot & \cdot & \cdot & 0 & 1 & 0 & \cdot & \cdot \\
\cdot & \cdot & \cdot & \cdot & \cdot & 0 & 0 & 0 & \cdot & 1 \\
\cdot & \cdot & \cdot & \cdot & 1 & 0 & 0 & 0 & 0 & 0
\end{array}\right] .
\end{aligned}
$$

In [15], Tsetlin was able to prove that his machine is $\epsilon$-optimal as per the definition of Eq. (2.6) where $c_{\min }<0.5$, and so:

$$
\lim _{n \rightarrow \infty} M(n)=\min \{c 1, c 2\}+\epsilon<0.5+\epsilon .
$$

and this is achieved by arbitrarily increasing the memory $N$, associated with the LA. 


\section{Krinsky Automaton $K_{1}$}

After Tsetlin proposed his $2 N$-state LA, different types of the similar $2 N$-state LA were introduced, including one due to Krinsky. Krinsky's LA design had two different mechanisms for each reward and penalty feedbacks. In Figure 2.3, we can see the transition functions for the Krinsky LA. When the machine receives a reward, the state changes from the current state to the most internal state i.e., $\phi_{i}(i=1,2, \ldots, N)$ to $\phi_{1}$, or from $\phi_{i}(i=N+1, N+2, \ldots, 2 N)$ to $\phi_{N+1}$, without going through any of the intermediate states. On the other hand, when it receives a penalty, it behaves just as the original Tsetlin's machine, $L_{2 N, 2}$, by going step-by-step towards the opposing action. It was shown that Krinsky's machine is $\epsilon$-optimal in all Random Environments.

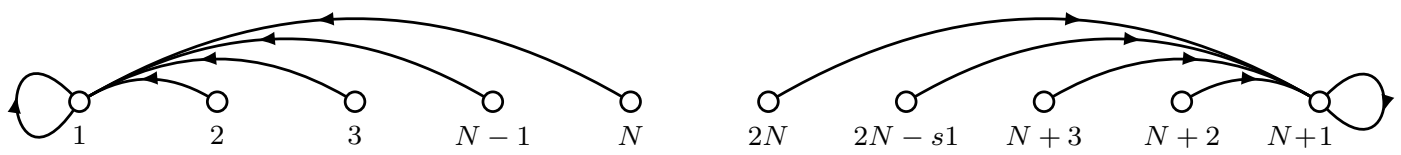

On a reward, $\beta=0$

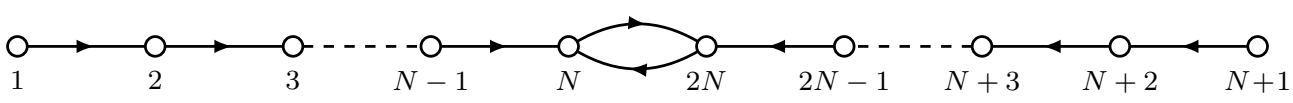

On a penalty, $\beta=1$

Figure 2.3: The state transition graphs for Krinsky's $K r_{2 N, 2}$.

For the Krinsky scheme, the transition matrix for a penalty response, $F^{(1)}$, is the same as in Tsetlin's case. On the other hand, the transition matrix for a reward response, $F^{(0)}$, is described as follows:

$$
\begin{array}{rlrl}
f_{i j}^{0} & =1 \quad \text { if } \quad i=1,2, \ldots, N & j=1 \\
& =0 . & & j \neq 1 \\
f_{i j}^{0} & =1 \quad \text { if } & i=N+1, N+2, \ldots, 2 N & j=N+1 \\
& =0 . & & j \neq N+1
\end{array}
$$


For the Krinsky machine, the expected penalty for the LA can be shown to be as follows:

$$
M\left(K_{1}\right)=\frac{c_{1} c_{2}^{N}+c_{2} c_{1}^{N}}{c_{1}^{N}+c_{2}^{N}}=\frac{\frac{1}{c_{1}^{N-1}}+\frac{1}{c_{2}^{N-1}}}{\frac{1}{c_{1}^{N}}+\frac{1}{C_{2}^{N}}},
$$

and so, as $N \rightarrow \infty$, we have:

$$
\lim _{n \rightarrow \infty} M\left(K_{1}\right)=\min \{c 1, c 2\} .
$$

Hence, the Krinsky machine is $\epsilon$-optimal in all Random Environments.

\section{Krylove Automaton $K_{2}$}

A subsequent work was done by Krylov which has also been described in [8]. In Figure 2.4, we can see the transition function of the Krylov machine and how it behaves. His LA works as follows; When the LA receives a reward, it responds the same way as in Tsetlin's $L_{2 N, 2}$. On the other hand, when it receives a penalty, it moves with a probability $\frac{1}{2}$ as if it was a $L_{2 N, 2}$ penalty and a probability $\frac{1}{2}$ as if it was a $L_{2 N, 2}$ reward, i.e., from state $\phi_{i}(i \neq 1, N, N+1,2 N)$ to $\phi_{i+1}$ with probability $\frac{1}{2}$ and to $\phi_{i-1}$ with probability $\frac{1}{2}$. When $i=1, N+1$, the LA stays in the same state with a probability of $\frac{1}{2}$ and can transition to $\phi_{i+1}$ with a $\frac{1}{2}$ probability. When $i=N$, the current state moves to $\phi_{N-1}$ with a $\frac{1}{2}$ probability or can move to $\phi_{2 N}$ with the same probability. Finally, when $i=2 N$, the current state moves to $\phi_{2 N-1}$ with probability of $\frac{1}{2}$ or it can move to $\phi_{N}$ with the same probability.

Thus, we can formulate Krylov's transition matrix when there is a penalty as follows:

$$
\begin{array}{ccl}
f_{i, i-1}^{1}=f_{i, i+1}^{1} & =\frac{1}{2}, \quad(i \neq 1, N, N+1,2 N) \\
f_{1,1}^{1}=f_{1,2}^{1} & =\frac{1}{2} ; \quad f_{N, N-1}^{1}=f_{N, 2 N}^{1}=\frac{1}{2} \\
f_{N+1, N+1}^{1}=f_{N+1, N+2}^{1} & =\frac{1}{2} ; \quad f_{2 N, 2 N-1}^{1}=f_{2 N, N}^{1}=\frac{1}{2} .
\end{array}
$$

For the Krylov LA, the asymptotic expected penalty can be shown to be as follows:

$$
M\left(K_{2}\right)=\frac{\frac{1}{\lambda_{1}^{N-1}} \frac{\lambda_{1}^{N}-1}{\lambda_{1}-1}+\frac{1}{\lambda_{2}^{N-1}} \frac{\lambda_{2}^{N}-1}{\lambda_{2}-1}}{\frac{1}{c_{1}} \frac{1}{\lambda_{1}^{N-1}} \frac{\lambda_{1}^{N}-1}{\lambda_{1}-1}+\frac{1}{c_{2}} \frac{1}{\lambda_{2}^{N-1}} \frac{\lambda_{2}^{N}-1}{\lambda_{2}-1}},
$$



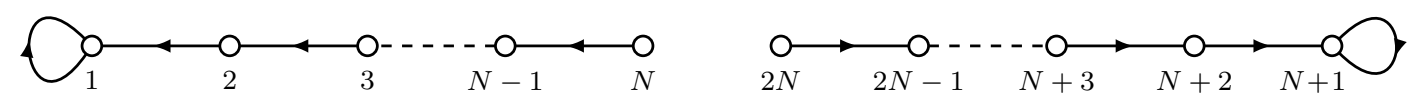

On a reward, $\beta=0$

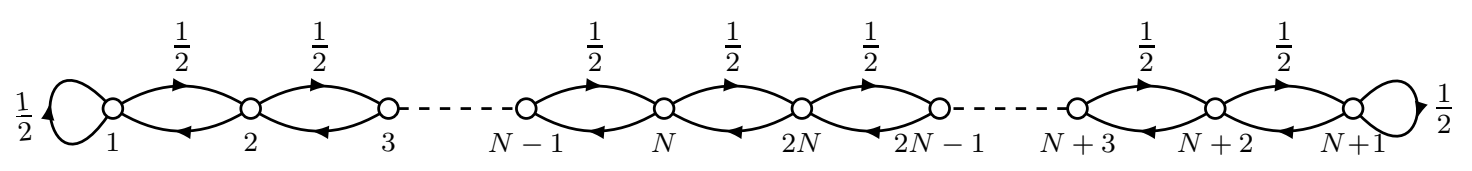

On a penalty, $\beta=1$

Figure 2.4: The state transition graphs for Krylov's $L_{2 N, 2}$.

where $\lambda_{\alpha}=\frac{c_{\alpha}}{1+d_{\alpha}}$, and $d_{\alpha}=\left(1-c_{\alpha}\right),(\alpha=1,2)$. Thus, as $N \rightarrow \infty$ :

$$
\lim _{n \rightarrow \infty} M(K r y)=\min \{c 1, c 2\}+\epsilon,
$$

where this is achieved by arbitrarily increasing the memory N, associated with the LA. Krylov's automaton has also been proven to be $\epsilon$-optimal in all Random Environments.

\subsubsection{Variable Structure Stochastic Learning Automata (VSSA)}

In VSSA, the action and the transition probabilities are not constant, as in the case of FSSA. Rather, they could change at every time instant. The action probabilities are, consequently, affected by these changes, and the theory of Markov Processes is necessary to analyze VSSA.

VSSA were first proposed by the researchers Varshavskii and Vorontsova in [38] by introducing the first automata that updated its transition probabilities. To simplify the mathematical expressions, we note that each state corresponds only to a single action. Hence we have $r=s<\infty$, and $G$, for VSSA, to be the identity mapping. From this, we can represent an automaton in the case of VSSA as a triple:

$$
\{\underline{\alpha}, \underline{\beta}, A\}
$$


where $A$ is the reinforcement scheme or the learning algorithm, and the other elements of the triple are the same as mentioned in the previous section. The action probabilities of the VSSA are updated as they interact with the Random Environment.

A general reinforcement scheme, which is used to update the action and state probabilities, can be represented by either one of two representations. Firstly,

$$
P(n+1)=T[P(n), \alpha(n), \beta(n)],
$$

where $T$ is a mapping. In the above equation, Eq. (2.19), the updated action probability vector at time $n+1, P(n+1)$, is based on the probability vector at time $n, P(n)$, the action taken at time $n, \alpha(n)$, and the Environment's response, $\beta(n)$. Alternatively, it can also be represented by

$$
f_{i j}^{\beta}(n+1)=T^{\prime}\left[f_{i j}^{\beta}(n), \phi(n), \alpha(n), \beta(n)\right],
$$

where $T^{\prime}$ is a mapping. In Eq. (2.20), $f_{i j}^{\beta}$ is the transition probability of moving from state $\phi_{i}$ to $\phi_{j}$ at time $n$ given that the Environment's response is $\beta(n)$. Typically, Eq. (2.19) is used to characterize VSSA rather than Eq. (2.20). If $P(n+1)$ is a linear function of $P(n)$, the scheme is referred to as being linear, otherwise it is non-linear.

In general, reinforcement schemes can be classified based on three criteria:

- Their asymptotic behaviour (i.e., whether they are expedient, absolute expedient or $\epsilon$-optimal)

- The nature of $T$ or $T^{\prime}$ (i.e., whether they are linear or non-linear)

- The properties of the underlying Markov process (whether it is ergodic or absorbing).

Different types of mappings have been proposed in the literature. Linear schemes have been the most extensively-studied schemes due to their analytical tractability. Before we specify the families of linear schemes, we will first present the general scheme, and then see how they simplify to linear schemes. 
In [15], the general updating scheme for the action probabilities was represented as follows. If $\alpha(n)=\alpha_{i},(i=1,2, \cdots, r)$, the updating rules for the cases when $\beta=0$ and $\beta=1$ are as given by Eq. (2.21) and Eq. (2.22) respectively.

$$
\begin{array}{ll}
p_{j}(n+1)=p_{j}(n)-g_{j}[P(n)] & \text { when } \beta(n)=0, \\
p_{j}(n+1)=p_{j}(n)+h_{j}[P(n)] & \text { when } \beta(n)=1, \forall j \neq i .
\end{array}
$$

Since $\sum_{j=1}^{r} p_{j}(n)=1$, this implies that:

$$
\begin{aligned}
& p_{i}(n+1)=p_{i}(n)+\sum_{j=1, j \neq i}^{r} g_{j}[P(n)] \quad \text { when } \beta(n)=0, \\
& p_{i}(n+1)=p_{i}(n)-\sum_{j=1, j \neq i}^{r} h_{j}[P(n)] \quad \text { when } \beta(n)=1 .
\end{aligned}
$$

For the functions $g_{j}$ and $h_{j}(j=1,2,3, \cdots, r)$ in Eq. (2.21) and Eq. (2.22), one observes that they must follow the following:

Assumption 1: $\forall j, g_{j}$ and $h_{j}$ are continuous functions;

Assumption 2: $\forall j, g_{j}$ and $h_{j}$ are non-negative functions;

Assumption 3: $0<\sum_{j=1, j \neq i}^{r}\left[p_{j}(n)+h_{j}[P(n)]\right]<1$.

The above must be true for all $i=1,2,3, \cdots, r$, and for all $P(n)$ for which its elements are in the open interval $(0,1)$.

In linear schemes, there are three main possibilities, as discussed in [15]. These are the Linear Reward-Penalty scheme $\left(L_{R-P}\right)$, the Linear Reward- $\epsilon$-Penalty scheme $\left(L_{R-\epsilon P}\right)$, and the Linear Reward-Inaction scheme $\left(L_{R-I}\right)$. We discuss below the two main schemes, which are the $L_{R-P}$ and the $L_{R-I}$.

\section{Linear Reward-Penalty $\left(L_{R-P}\right)$ Scheme}

One of the earliest schemes that were introduced was the $L_{R-P}$ scheme. It was studied in details by the researchers in [38]. In the $L_{R-P}$, once the Environment sends the feedback to the LA, the LA uses this feedback to increase the probability of the selected action and reduce the others when it receives a reward. On the other hand, when it receives a penalty, it decreases the selected action's probability and possibly increases the probabilities of the other actions. In [10], McMurtry and Fu implemented and studied the model, in detail, for continuous data. 
If we consider the $r$-action LA, we can specify Eq. (2.21) and Eq. (2.22) by

$$
\begin{array}{ll}
g_{j}[P(n)]=a p_{j}(n) & 0<a<1, \quad \text { and } \\
h_{j}[P(n)]=\frac{b}{r-1}-b p_{j}(n) & 0<b<1,
\end{array}
$$

where, in the above, $a$ and $b$ are the reward and penalty parameters respectively.

Thus, the $L_{R-P}$ can represent the updating algorithm as follows:

If $\alpha(n)=\alpha_{i}$,

$$
\left.\begin{array}{l}
p_{i}(n+1)=p_{i}(n)+a\left[1-p_{i}(n)\right] \\
p_{j}(n+1)=(1-a) p_{j}(n), j \neq i
\end{array}\right\} \quad \beta(n)=0,
$$

When $a=b$, the $L_{R-P}$ is at most expedient, implying that it is "better than nothing". On the other hand it is $\epsilon$-optimal when $b \ll a$, and when $a \rightarrow 0$ simultaneously, leading to the $L_{R-\epsilon P}$ scheme.

\section{Linear Reward-Inaction $\left(L_{R-I}\right)$ Scheme}

Another scheme was introduced subsequently by the researchers Shapiro and Narendra in [26]. This scheme is based on the idea of ignoring the penalty when the LA receives one, implying that it maintains the action probabilities as they are. On the other hand, when it receives a reward, it changes the action probability as in the $L_{R-P}$. This modified scheme can be achieved by setting $b=0$ in Eq. (2.25). Hence, it can be characterized by the following updating scheme:

If $\alpha(n)=\alpha_{i}$,

$$
\begin{aligned}
& \left.\begin{array}{l}
p_{i}(n+1)=p_{i}(n)+a\left[1-p_{i}(n)\right] \\
p_{j}(n+1)=(1-a) p_{j}(n), j \neq i
\end{array}\right\} \quad \beta(n)=0, \\
& p_{i}(n+1)=p_{i}(n) \quad \beta(n)=1 .
\end{aligned}
$$

The $L_{R-I}$ can be proven to be $\epsilon$-optimal. 


\subsubsection{Estimator and Pursuit Algorithms}

The concept of Estimator Algorithms was introduced by Thathachar and Sastry in $[34,35]$ when they realized that the family of Absolutely Expedient algorithms would be absorbing, and that they possessed a small probability of not converging to the best action. Estimator algorithms were initially based on Maximum Likelihood (ML) estimates (and later on Bayesian Estimates), where they also used the estimates of the reward probabilities to update the actions' probabilities. This concept was achieved by keeping track of the number of rewards received by the selected actions and by pursuing the ones with the superior estimates. By doing this, the LA converged faster to the actions that possessed the higher reward estimates.

The original Pursuit Algorithms are the simplest versions of those using the Estimator paradigm introduced by Thathachar and Sastry in [36]. These algorithms are based on the pursuit strategy where the idea is to have the algorithm pursuing the best-known action based on the corresponding reward estimates. The pursuit algorithms were proven to be $\epsilon$-optimal. After the initial family of Pursuit algorithms, the concept of designing discretized Pursuit LA was introduced by Oommen and Lanctot in [19]. These LA were also proven to be $\epsilon$-optimal. The discretized versions of LA were shown to converge faster than their continuous counterparts.

\subsubsection{LA for Non-Stationary REs}

The vast majority of algorithms discussed in the previous sections were designed for stationary Environments, where the penalty probabilities do not change with time. Unfortunately, in many real life scenarios, this is not the case. There have thus been approaches to formulate and design LAs for non-stationary REs, where the penalty probabilities that are associated with the actions change with time. Although, in our work, we consider only the stationary case for Elevator-like problems, this section is included primarily for the sake of completeness.

In Non-Stationary Environments, there is no fixed best or optimal action. Rather, 
the LA attempts to find the action that minimizes the time-averaged expected penalty:

$$
\lim _{T \rightarrow \infty} \frac{1}{T} \sum_{n=1}^{T} E[\beta(n)] .
$$

There are many types of non-stationary Environments that LA can work with. However, three of the main cases are:

- Markovian Switching Environments (MSEs);

- Periodic Environments (PEs);

- Hierarchical Systems.

In the first case, the MSEs, the Environments themselves are considered to be states of a Markov Chain. Different schemes, FSSA and VSSA, which interact with such MSEs, were discussed in [14]. In the case of PEs, the operation of LA was introduced by Narendra and Viswanathan in [16], where the Environment changed periodically. In their LA, the design included two levels of automata. The purpose of the first level was to learn the periodic behaviour of the Environment, while the second level learned the best action for that specific environment. Poznyak introduced the concept of hierarchical LA systems in [24] where the paper proposed the design of LA that operated in a two-level scheme. Subsequently, hierarchical tree-based LA systems were proposed by Thathachar and Ramakrishnan in [33].

Baba and Sawaragi presented an alternate result for non-stationary Environments in [1] where the $L_{R I}$ scheme was proven to be $\epsilon$-optimal.

\subsubsection{Conclusions on the Review of LA}

The sections of this chapter surveyed a field of computer science which focuses on Learning. We started by addressing the definition of "Learning" and how it has been applied in various areas of computer science.

After this, we concentrated on the main area of interest, namely the field of Learning Automata (LA), and showed how it is related to the concept of Learning. We then classified LA into different classes according to their characteristics. This 
involved into two main classes. The first was the family of Fixed Structure Stochastic Automata (FSSA), and the second was the family of Variable Structure Stochastic Automata (VSSA). We explained each class in detail and showed the differences between them. We also went through the performance and convergence phenomenon of each of the LA that we visited.

Subsequently, after introducing the concept of the Maximum Likelihood Estimator (MLE) of the actions' reward probabilities, we showed how LA can be enhanced to yield the families of Estimator and Pursuit LA. Finally, we mentioned the concept of non-stationary REs, and explained how they differed from the stationary ones. Here, we also mentioned the different types of non-stationary REs, i.e., Markovian, Periodic and Hierarchical systems.

In the following sections and chapters, we are going to introduce Elevator-Like Problems, and will describe how others have attempted to tackle this problem from different perspectives.

\subsection{Elevator-like Problems}

\subsubsection{Introduction}

Although the primary intent of this thesis is to study ELPs, it turns out that most of the state-of-art deals with concepts and solutions for real-life elevators. When it concerns solutions to each real-life elevator problem, these are usually concerned with optimizing the metrics for its efficiency. Some of these metrics are the waiting time, energy consumption or travel time. In this section, we will survey solutions to optimizing the motion of elevators and consider how it is formulated for both single and multiple elevators systems. We will then proceed to discuss different types of ELPs themselves.

First of all, we will present the single elevator problem. We will discuss attempts that have been made to solve it and consider the criteria used for the respective solutions. Thereafter, we will generalize the formulation to cover the multi-elevator problem and discuss the differences encountered when dealing with elevators in stationary 
and non-stationary environments. The reader will observe that the performance and solutions of the problem get complicated with the introduction of non-stationary environments.

We will conclude this survey by discussing some ELPs and how they are similar to the problems involving real elevators.

\subsubsection{Single Elevator Problem}

We first discuss the Single Elevator Problem (SEP) and explain the solutions by which researchers have tackled it in the past. As mentioned, the SEP is a subclass of the MEP in which we have a building with $n$ floors and a single elevator, and where we are to design a policy for the elevator in order to operate in such a way so as to save energy, reduce the travel time or the waiting time for passengers. Moreover, the elevator policy should decide how the elevator should operate in order to achieve its goal. For example, one possible policy could require that the elevator picks the best route in order to pick passengers, while another could be to serve the longest queue first or to decide where the elevator should wait for the next call. As we shall see, the literature does not record several solutions for the SEP. The reason for this is that most papers deal with the more complex scenario, the MEP, which is a generalization of the SEP.

Two notable contributions for the SEP were by Tanaka et al. in [30, 31]. In their papers, they studied the operation problem of a single-car elevator with so-called "destination hall call registration". "Destination hall call registration" is a system that is responsible for passengers registering their destination floors at the hall before boarding the elevator. This is opposed to regular or "traditional" elevators, where passengers can only pick the direction of their trip before boarding, and thereafter specify the destination floor after boarding. In the first part of their study [30], the authors were trying to answer two questions, namely: (a) "Can (single) car operations be improved with destination hall call registration?", and (b) "How can it be realized?". They were able to formulate the operation problem as a Dynamic Optimization Problem (DOP) such that an objective function, which is the weighted 
average waiting time for passengers, is minimized. This set the stage for the second question. For the first question, they were able to show by simulation that the DOP substantially improved the capability of the transportation when compared to the conventional "selective collective" operation.

In the second part [31], the authors introduced a branch-and-bound algorithm to solve the formulation of the DOP problem, which was the exact formulation proposed for the work in [30]. In this case, the lower bound calculations for the subproblems, that were generated in the course of the branch-and-bound algorithm, came from decomposing the problem into three subproblems, i.e., the passenger loading and unloading, car stops and lastly the car floor-to-floor travel. They then applied the Lagrangian relaxation method to solve the overall problem. Unfortunately, the authors observed that their algorithm was not fast enough for real-life situations. To improve it, they had to take into consideration the constraints of the elevator's capacity.

After their previous studies in [30] and [31], the authors conducted a study in [29] where they tried to examine how one could improve the efficiency of a single elevator system with "destination hall call" considering the objective functions that had to be dynamically optimized. In this paper, the authors applied a simulated annealing-based method, and from their results they showed that the weighted average of two different objective functions, such as the weighted average service time and the maximum, yielded a better performance than using only a single objective function. Another point that they observed was that one had to choose the weights of the objective functions carefully because this choice significantly affected the results of the experiments. Moreover, these weights changed depending on the elevator's specific characteristics.

The authors of [28] addressed the goal of estimating the optimal values for the upper and lower bounds for the elevator scheduling problem, in which they assumed the availability of all the information about the passengers. Because of the large numbers of decision variables that had to be taken into consideration, one could not compute the exact optimal performance, and they, thus, provided its estimate. To achieve this, they formulated the problem in two parts, a namely a high level and a low level components. The high level component was a passenger-to-car assignment, 
and the low level component was the passenger-to-trip assignment. In both these assignments, they defined a "trip" as the start of the elevator's movement in a single direction until the elevator reversed to go to the other direction. This then was used for the formulation of the low-level component. The authors obtained the upper bound, by finding a reasonable solution to the problem. They then obtained the lower bound, by defining a lower bound for a newly constructed similar problem using the Lagrangian Relaxation method. This method permitted the approximation of a constrained optimization problem by a more straightforward problem. Moreover, the solution to the simpler problem provided an approximation to the original problem. The results that the authors obtained were both efficient and scalable.

A subsequent work was done by Molina et al. [13], where they designed an algorithm that is based on Ant Colony Optimization (ACO) for solving the SEP. The ACO model involved a partially-connected construction graph used by the artificial ants to construct the solutions to the SEP. It provided a sequence of visits to the requested floors to minimize the average waiting time for the passengers. They introduced an objective function that specified how good the proposed solution was and the problem was formulated as a combinatorial optimization problem. Using the latter, the authors were able to minimize the average waiting time for passengers by invoking straightforward well-known ACO methods. In their experiments, they focused on finding the best parameters for their algorithm, which they referred to as the ACS-elevator to obtain the best sequence of visits for the elevator. These parameters were the initial positions of the elevator, the maximum capacity of the elevator, the number of floors in the building, the length of the sequence of visits or the solution, the maximum number of algorithm iterations, etc.

Another work was presented by $\mathrm{Xu}$ and Feng [39] modelled the single elevator scheduling problem as a mixed integer linear program (MILP). They focused on using this model to improve the service time for passengers. Based on a prototype model obtained from the industry for the dynamics of a moving elevator, they linearized the nonlinear travel activities for the problem. They also cut planes and introduced many constraints to accelerate the computation for solving the MILP. They tested their model under different circumstances and scenarios. After introducing their 
constraints, they observed that the problem converged in a radically short time. They conjectured that their model could be extended and used as a benchmark because of its simple implementation and the speed of the relevant computations.

\subsubsection{Multiple Elevator Problem}

In the previous section, we discussed the SEP and how researchers have attempted to solve it. In this section, we will consider an extension for the SEP which is the MEP. In the MEP, the problem is to try to optimize a set of elevators in a building instead of having only a single elevator. This extension, obviously, introduces more complexity to the problem and adds more constraints. The problem can be solved in two different types of environments. In the first case, we deal with a stationary environment, where the environment does not change with time. In the second scenario, the environment is non-stationary, and it changes with time. Here, the search space changes while one attempts to find the optimal solution to the problem. The solution could consist of a set of movements for the elevator, or finding the optimal parking floors where the elevators should be parked to minimize the service time or the waiting time for passengers.

In Crites and Barto's paper [2], they proposed a Reinforcement Learning (RL)based solution to the elevator dispatching problem. At the time of their work, there were a variety of challenges related to this problem that had not been addressed earlier. In the elevator system that they examined, they simulated a building with 10-stories and four elevators. They used a Poisson distribution for the arrival of passengers at each floor. They then modelled the system as a discrete event system where the events happened at discrete time instances. They also used a different RL agent for each elevator. Such a design made them encounter a couple of complications such as the noise that the agents obtained when they received a reinforcement signal because of the effects of other agents. After they conducted their experiments, they compared their results with eight previous algorithms and heuristics, and were able to demonstrate, by their simulations, that their algorithm noticeably outperformed the previous algorithms. 
In [5], Cho et al. proposed a new approach for the control system for a group of elevators. To estimate the waiting time for hall calls, they formulated two dominant parameters, which were the stop number and the travelling distance. They showed that their estimation was valid for different traffic patterns such as up-peak, downpeak and inter-floor time using computer simulations on a twenty-floor building that had three elevator cars. To deal with exceptional situations and a load of passengers in the cars, they used two assignment policies for the elevator cars. The first one was a No-Cancellation Policy, which meant that cars that had already been assigned to a hall call could not be re-assigned. The second one involved a Re-Assignment Policy, and this allowed the re-assignment of hall calls to another car after they had been assigned a specific car. They also introduced the concept of extended policies for these two scenarios that additionally took into consideration threshold times referred to as NCP-THT and RAP-THT, specifically introduced to reduce the variance of hall wait times.

In $[17,40]$, the authors presented an algorithm to calculate estimates for the average waiting time for passengers in group elevator systems. They modelled the system as a discrete-state Markov chain for the moving elevator car. They thus used dynamic programming to analyze the Markov Chain and to compute the expected measures for their proposed metrics for the performance of the elevators. These metrics were, for example, the expected average waiting time for the passengers. They then introduced a linear-time controller that was based on the method that they had earlier specified, referred to as the ESA-DP algorithm.

In their paper, they first introduced the "base" ESA-DP scheme after which they extended the algorithm to deal with specific cases. Their base algorithm involved some assumptions such as an unlimited elevator capacity, no future passenger arrivals, full state information and a known marginal distribution for the destination floors. In the subsequent work, they extended the algorithm to deal with the nonuniform distributions for the destination floors. They then showed, in their results, that this algorithm outperformed other algorithms that were used as benchmarks and that they were able to reduce the waiting times by $30 \%-40 \%$ in heavy traffic scenarios. Besides, their algorithm was applicable and computationally efficient for real-life 
elevator systems.

In [4], which might be considered to be the closest representation and approach to the model and solution that we propose, the authors presented a paper concerning the problem of optimally parking elevator cars in a building with an attempt to minimize the waiting time for passengers. This was done by anticipating the arrival of the passengers. They studied two traffic patterns of passengers which involved the down-peak and up-peak settings. These patterns represent the cases when the frequency of passengers going down to the lobby is high, and alternatively when the frequency of going up the building from the lobby was high, respectively. The authors of [4] modelled the system as a Markov Decision Process whose states corresponded to possible parking locations or floors. Using this, they proposed two dynamic programming solutions for the elevators for selecting which location they should park the cars for the up-peak and down-peak traffic patterns. The results that they obtained demonstrated that their algorithm was able to acquire the dependency between the number of cars parked that needed to be parked at the lobby and the arrival rate of passengers. It also yielded good results for the medium and low arrival patterns.

In [42], the authors presented an algorithm that combines RL and Neural Network to achieve a better performance for group elevator systems. They first formulated the model of the group elevator system as an MDP. They then defined the elements of the model based on their algorithm. They considered metrics such as the riding time, the waiting time for the passengers and the task of reducing the number of stops. They thus defined their goal to find an optimal dispatching policy that minimizes these metrics for the overall elevator system. To be able to pick the best dispatching policy, they applied a stochastic exploration strategy and the neural network with back propagation together with a Q-learning algorithm. They used Q-learning to approximate the state-value function. They then used a Gibbs distribution for the selection of elevators to serve new passengers. The authors also simulated the behaviour of their algorithm on a building with four elevators and with different traffic patterns. From the results that they obtained, they were able to compare the performance of their algorithm with a GA-based scheme. They demonstrated that their algorithm drastically reduced the waiting time and average riding time when compared to the 
GA algorithm.

Similar to [4], the authors of [9] presented an optimal parking strategy for the MEP. However, in this case, they focused on saving energy and how it affected the trip time, i.e., whether it would be reduced or not for the group elevator systems during inter-floor traffic patterns. They first constructed the model of the energy consumption for the elevator system and acquired the data that corresponded to it. They then developed a GA to find the optimal solution, which was the parking zones for the elevator cars. They then simulated the algorithm on a 13-floor building with three elevator cars. The results that they acquired demonstrated that by finding optimal or close-to-optimal parking zones, reduced the energy consumption and waiting time for passengers significantly by $10.38 \%$ and $41.55 \%$ respectively compared to the common zoning strategy.

In [3], the authors proposed a new algorithm also based on GA and Tabu Search for the elevator group control problem. They were the first to introduce the concept of Tabu search for this problem domain. They invoked the two approaches to maximize the efficiency of hall calls and to reduce the waiting time for the passengers. They first defined the mathematical formulae and models for the elevator system, and defined the average journey time (AJT), which consisted of the average travel time and the average waiting time. They then introduced their GA algorithm and Tabu search methods for the controller. They tested their algorithms using computer simulation in different scenarios for buildings having 10 to 24 floors and a different number of elevator cars, i.e., from 2 to 6 cars. They also tested lunch-peak traffic since it included up-peak and down-peak patterns. From the results that they obtained, they showed that the GA outperformed the conventional methods used in group elevator systems, and that it reduced the average waiting and journey time for the passengers while maintaining a reasonable computational effort that allowed it to be implemented in real elevators. On the other hand, the Tabu search results were characterized by worse times when compared to the GA's performance, but they believed that it could also be implemented in real elevators.

Another work that is based on RL was proposed by the authors of [41]. They presented an algorithm based on RL and specifically on Q-learning to find the optimal 
dispatching policy to optimize the average waiting time and average riding time for the passengers in a group elevator system. They first described the mathematical model that they constructed and used MDP for representing the system. They then presented the RL algorithm that they used, which was based on neural networks that estimated the criterion functions. The authors conducted simulations to test their algorithm on a 20-floor building with five elevator cars. The results that they acquired were compared with two other algorithms, the first being GA-based and the other using Temporal Differences. The authors of [41] showed that their solution was feasible and that it possessed an average performance comparable to these methods, but which was superior to a GA-based solution during peak times.

Another approach for optimizing the average waiting time for group elevator systems was mentioned in [6], where the authors used a fuzzy logic-based algorithm. Their algorithm was based on dynamic dispatching, which allowed the elevators to be reassigned to a hall call. They first compared the differences between using a non-dynamical fuzzy logic dispatching design and a dynamical fuzzy logic dispatching through feedback. This feedback was the fundamental concept for the design, because this was how the parameters for the fitness function were updated with every iteration. They simulated their algorithm on a 19-floor building with seven elevator cars. Their simulations had two traffic patterns, namely down-peak and inter-floor traffic. They then compared their results to the "nearest call algorithm", which is the most commonly-used dispatching algorithm in the industry. The results that these authors obtained demonstrated that their algorithm decreased the average waiting time drastically.

In [32], the authors proposed new GA-based algorithms to optimize the dispatching of group elevator systems to reduce the car trip time, waiting time, travel time and the journey time for the passengers. The authors first encoded the problem so as to be able to solve it using GAs. They then proposed their algorithms where they introduced two different schemes for two different scenarios. The first scenario was when the system did not know the destination of the passengers, which meant that passengers only called the elevator and they did not specify the destination floor until they entered the car. Instead, they specified only the direction of their proposed trip. 
In this scenario, they tried to optimize the waiting time for passengers. The other scenario was when the passenger could specify the destination floor from the hall call before boarding the car. For this scenario, they proposed a second GA to optimize the waiting time, car travel time and the journey time. The authors simulated their algorithms on a 20-floor building which consisted of 4 elevator cars, and compared their results with two GAs and the conventional duplex algorithm. The results that they obtained demonstrated that their algorithms outperformed the previous GAs against which they had done the comparison.

In [27], the authors presented a different research task than what had been undertaken in the previous papers, where they focused more on the waiting time for passengers' queues. They first analyzed the operation of the elevator system so as to understand how it operated. They then tried to minimize the size of the waiting passengers' queues. They divided the elements into two groups, namely passive and active ones, which were the queues and the processors or the servers respectively. They then developed a simulation for these elements that enabled them to test the model and the configuration of the system. This enabled them to use a heuristic to find the best configuration suitable for the elevator to minimize the queues' waiting time. Their simulations consisted of different scenarios by using six and seven elevators in a 12 -floor building. The results that they obtained from their simulations demonstrated that the waiting time for passengers reduced by $13 \%$ for the six-elevator scenario, and by $37.08 \%$ in the seven-elevator scenario.

\subsubsection{Elevator-like Problems}

It is noteworthy that solutions to problems involving Elevators are also pertinent to Elevator-like Problems, i.e., other problems that are "similar" to the elevator problem. Indeed, they can be seen to share the same mapping and analogous optimization conditions. These are, generally, optimization problems, and are specifically problems where the agent tries to stay idle in a place waiting for the next anticipated location [31] which may not necessarily be a "floor" in a building. 
One example of this type of problem in two dimensions is the problem of a tennis player moving to a location waiting for the return shot. Another example is the problem of parking of police cars and emergency vehicles, where the parking location best anticipates the next location where they are needed. A third analogous problem involves locating the position of the read/write head for computer disks. Other potential tasks could be those of locating security guards (or alarms) in buildings so as to have their optimal physical distributions [22]. A similar problem, studied by Porretta [23], involves improving underwater communication systems that learn to adapt by knowing where to locate the underwater sensors.

All these problems share the same underlying structure (or mapping), and we seek a standard mechanism for their common solution. These problems differ in the objective functions that quantify the qualities of the solutions, i.e., of how good the solutions or the chosen actions are. In spite of the above, in the interest of simplicity, in this thesis, we consider the physical elevator problem and attempt to use the passengers' waiting time as the metric to measure the performance of a potential solution.

\subsection{Chapter Conclusion}

In this chapter, we first introduced the concept of learning from a philosophical perspective. We then introduced the paradigm of Learning Automata (LA) which uses the concepts of learning and which has been reckoned as a sub-field of reinforcement learning. The summary of what we considered is given in Section 2.2.10.

In the second half of the survey, we surveyed the Elevator Problem and Elevatorlike Problems. As explained, most of the work done on the Elevator problem is related to obtaining a control policy and of how one can design efficient policies. As far as we know, there is only a single paper that was close to our goal, namely, that of determining the optimal parking position for the elevator so as to reduce the waiting time for the passengers. In our work, we will try to achieve an LA-based near-optimal strategy for parking single and multiple elevators so as to reduce the passengers' waiting time. 


\section{Chapter 3}

\section{Solutions for the SEP}

\subsection{Introduction}

In this chapter, we will first introduce the "competitive" models that we will use for the SEP against which we will compare the performance of our proposed LAbased solutions. In each case, we will present the policy used and the results of the simulations of these models that will be recorded as benchmarks. Apart from considering SEP models, we shall also highlight their salient features. We will then describe how LA have been used by us to resolve the SEP, and also submit the simulation results for the new solutions.

In the literature, not many of the reported papers have tackled the SEP since most of the researchers considered the more complicated problem, namely, the MEP. Thus, there were only two solutions that we considered as being competitive. The first solution, referred to as SEP1, is one where the elevator parks at the same floor as the current destination i.e., the drop-off location of the passenger call. We believe that it is the most widely-used policy. The second, referred to as SEP2 [22], moves the elevator car and parks it at a predetermined destination after every passenger drop-off. 


\subsection{Models of Computation}

There are essentially two models of computations that can be envisioned when we attempt to solve the SEP. The first assumes that the distributions at stake, namely the pick-up and the destination distributions, are known ${ }^{1}$ a priori. By virtue of this prior knowledge, one can calculate the optimal parking floor.

On the other hand, unarguably, the more complex scenario involves the model in which the pick-up and destination distributions are unknown, and not estimated either. Solutions for this model will utilize LA, as explained later in this chapter.

The LA-based solutions do not require any information about the calling or dropoff distributions. They are "unknown" to the decision-making algorithm, which calculates the best parking floor. Instead, they are "blindfolded", and they only know how the Environment (the overall elevator system) responds upon receiving instructions as to where the car should be parked.

To formalize this, we assume that there are two pertinent distributions, $\mathcal{C}$ and $\mathcal{D}$. $\mathcal{C}=\left\{c_{1}, \ldots, c_{n}\right\}$ specifies the probabilities of passengers requesting to be picked up, where $c_{i}$ is the probability that a passenger requests a pickup from floor $i$. Similarly, $\mathcal{D}=\left\{d_{1}, \ldots, d_{n}\right\}$, where $d_{j}$ specifies the probability that a passenger will alight at floor $j$. Our computation model assumes that $\sum_{i=1}^{n} c_{i}=1$ and $\sum_{j=1}^{n} d_{j}=1$. Throughout our study, we will assume that the elevator car carries only a single passenger ${ }^{2}$ at a time and that this passenger is serviced before the subsequent passengers' requests are processed. Of course, the elevator car could pick up a passenger while it is on its route from the calling floor to the destination floor. That being stated, this is not primarily important in our study, because we are more concerned with where the car will be parked during the idle period. Observe that the Environment that determines where the passenger issues his calls from and where he goes to has to be aware of the distributions $\mathcal{C}$ and $\mathcal{D}$. However, this knowledge may not be available to the algorithms that deal with planning. In the first model of computation stated above, these distributions are known to the planning algorithm. They are unknown in the second model described above.

\footnotetext{
${ }^{1}$ If they are not known, we can assume that they are obtained by a pre-processing estimation.

${ }^{2}$ The multi-passenger case is currently open.
} 


\subsection{Simulation Settings}

Simulations require mutual specific configurations or settings so as to be able to compare the different results obtained from different solutions. In our simulations, we will incorporate different simulation settings for the buildings. The first item for these settings is the number of floors that the building has. In the interest of uniformity, we will test the models on four different types of buildings with varying numbers of floors, which are 8-floor, 12-floor, 16-floor and 20-floor buildings.

We will also perform the simulations using four different types of distributions ${ }^{3}$ for $\mathcal{C}$, listed below:

1. The first is an exponential distribution designed, referred to as Exp, to simulate an up-peak traffic pattern, where most calls come from the ground and/or lower floors, and where the passengers intend to travel up;

2. The second distribution is the inverse exponential distribution, referred to as InvExp, that represents a down-peak traffic pattern, where most calls are from the upper floors, and where the passengers travel downward;

3. The third distribution is the Gaussian (Normal) distribution, referred to as Gaussian, and this is intended to represent the traffic of passengers during the middle of the day, also referred to as "regular traffic";

4. The final distribution that we will use is a bimodal distribution, referred to as Bimodal, which is specified as a mixture of Gaussian distributions to represent a more complex "regular traffic" pattern.

Given a particular distribution for $\mathcal{C}$, we now specify how the discretized probabilities for the respective floors are obtained. We clarify this in Figure 3.1 for a 12-floor building with a bimodal distribution. The heights of the bimodal curve for the 12 uniformly placed points on the $x$-axis represent the corresponding probabilities. The final values of the probabilities are obtained by normalizing these heights so that the sum becomes unity.

\footnotetext{
${ }^{3}$ It is a trivial task to examine other distributions, for example, ones for which $c_{1}$ is close to unity, as in the real-life setting of early morning traffic.
} 


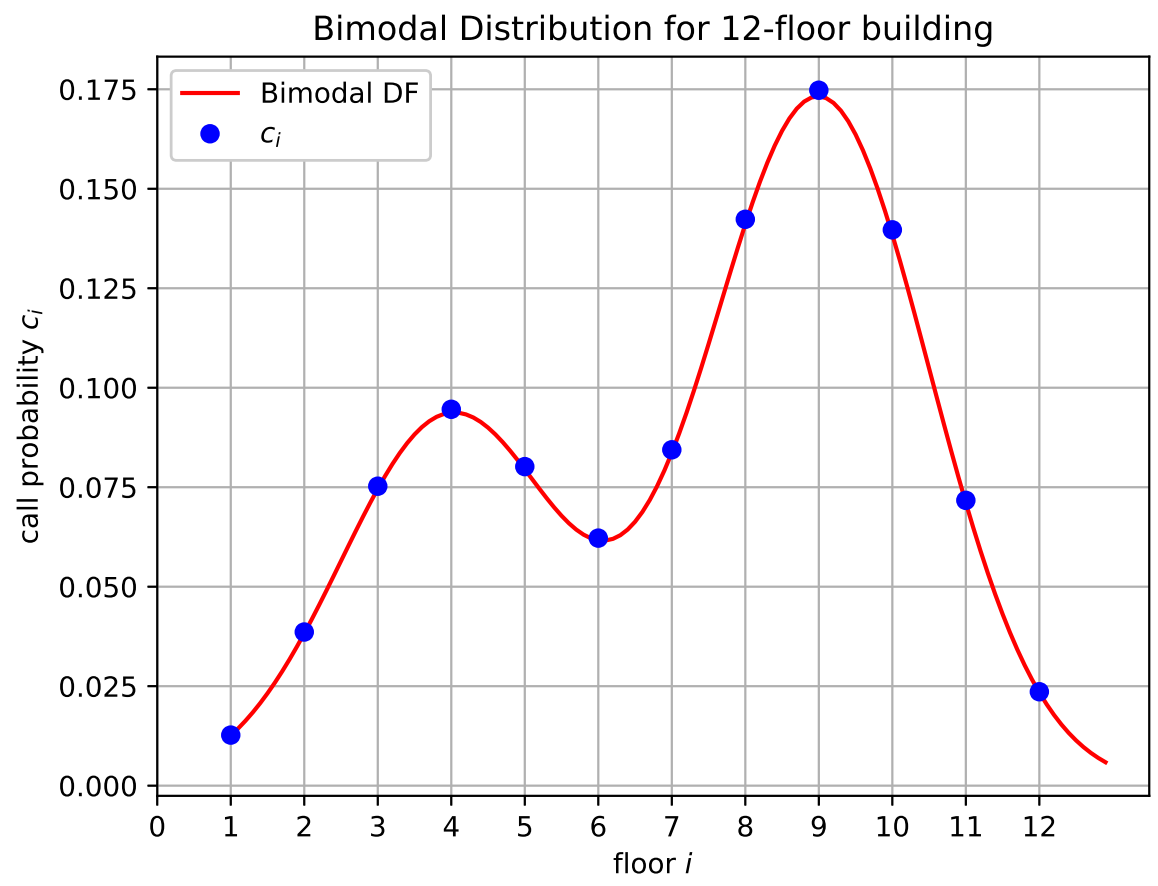

Figure 3.1: A typical bimodal distribution for a 12-floor building and the corresponding discretized probabilities constituting $\mathcal{C}$.

Tables 3.1, 3.2, 3.3 and 3.4 represent the calling probabilities obtained from the four different distributions mentioned above for 8, 12, 16 and 20-floor buildings respectively. The corresponding tables for the Destination distributions are obtained in an analogous manner.

\subsection{Previous Solutions}

As mentioned above, we first present the two solutions that we intend to use as benchmarks for the single-elevator scenario. The first policy is currently the one predominantly used in modern-day settings. The principle motivating this is that once the elevator picks up a passenger and drops him at his destination, it waits at the same floor for the next call - without attempting to move to an alternate, possibly superior, floor during the idle period. The second policy uses the "known" 
Table 3.1: Simulation settings for $\mathcal{C}$ for an 8-floor building for different distributions. The values represent the probabilities of receiving a passenger call from each floor in accordance with the associated distribution.

\begin{tabular}{c|cccc}
\hline Dist & Exp & InvExp & Gaussian & Bimodel \\
\hline$c_{1}$ & 0.59387372 & 0.00109053 & 0.08215232 & 0.01898451 \\
$c_{2}$ & 0.24145104 & 0.00268228 & 0.1146528 & 0.08516571 \\
$c_{3}$ & 0.09816667 & 0.00659734 & 0.14318402 & 0.14316436 \\
$c_{4}$ & 0.03991159 & 0.01622684 & 0.16001087 & 0.12033347 \\
$c_{5}$ & 0.01622684 & 0.03991159 & 0.16001087 & 0.17698614 \\
$c_{6}$ & 0.00659734 & 0.09816667 & 0.14318402 & 0.2620605 \\
$c_{7}$ & 0.00268228 & 0.24145104 & 0.1146528 & 0.15804965 \\
$c_{8}$ & 0.00109053 & 0.59387372 & 0.08215232 & 0.03525567 \\
\hline
\end{tabular}

Table 3.2: Simulation settings for $\mathcal{C}$ for a 12 -floor building. The meaning of the entries is as described in the caption of Table 3.1.

\begin{tabular}{c|cccc}
\hline Dist & Exp & InvExp & Gaussian & Bimodal \\
\hline$c_{1}$ & 0.59344245 & 0.00002978 & 0.02592411 & 0.01270883 \\
$c_{2}$ & 0.24127569 & 0.00007324 & 0.04518336 & 0.03860891 \\
$c_{3}$ & 0.09809538 & 0.00018013 & 0.07046903 & 0.07525205 \\
$c_{4}$ & 0.0398826 & 0.00044306 & 0.09834746 & 0.09457958 \\
$c_{5}$ & 0.01621506 & 0.00108974 & 0.12282111 & 0.08017525 \\
$c_{6}$ & 0.00659255 & 0.00268033 & 0.13725493 & 0.06220755 \\
$c_{7}$ & 0.00268033 & 0.00659255 & 0.13725493 & 0.08440493 \\
$c_{8}$ & 0.00108974 & 0.01621506 & 0.12282111 & 0.14232761 \\
$c_{9}$ & 0.00044306 & 0.0398826 & 0.09834746 & 0.17475873 \\
$c_{10}$ & 0.00018013 & 0.09809538 & 0.07046903 & 0.13967666 \\
$c_{11}$ & 0.00007324 & 0.24127569 & 0.04518336 & 0.07169797 \\
$c_{12}$ & 0.00002978 & 0.59344245 & 0.02592411 & 0.02360195 \\
\hline
\end{tabular}


Table 3.3: Simulation settings for $\mathcal{C}$ for a 16 -floor building. The meaning of the entries is as described in the caption of Table 3.1.

\begin{tabular}{c|cccc}
\hline Dist & Exp & InvExp & Gaussian & Bimodal \\
\hline$c_{1}$ & 0.59343067 & 0.00000081 & 0.00588623 & 0.00309355 \\
$c_{2}$ & 0.24127091 & 0.000002 & 0.01281213 & 0.00952918 \\
$c_{3}$ & 0.09809343 & 0.00000492 & 0.02495463 & 0.02286339 \\
$c_{4}$ & 0.03988181 & 0.00001211 & 0.04349365 & 0.04274851 \\
$c_{5}$ & 0.01621473 & 0.00002978 & 0.06783372 & 0.06242093 \\
$c_{6}$ & 0.00659242 & 0.00007324 & 0.09466958 & 0.07186065 \\
$c_{7}$ & 0.00268028 & 0.00018013 & 0.118228 & 0.06788 \\
$c_{8}$ & 0.00108972 & 0.00044305 & 0.13212205 & 0.0604008 \\
$c_{9}$ & 0.00044305 & 0.00108972 & 0.13212205 & 0.06530901 \\
$c_{10}$ & 0.00018013 & 0.00268028 & 0.118228 & 0.08883733 \\
$c_{11}$ & 0.00007324 & 0.00659242 & 0.09466958 & 0.1184869 \\
$c_{12}$ & 0.00002978 & 0.01621473 & 0.06783372 & 0.13154 \\
$c_{13}$ & 0.00001211 & 0.03988181 & 0.04349365 & 0.1155474 \\
$c_{14}$ & 0.00000492 & 0.09809343 & 0.02495463 & 0.07933226 \\
$c_{15}$ & 0.000002 & 0.24127091 & 0.01281213 & 0.04245367 \\
$c_{16}$ & 0.00000081 & 0.59343067 & 0.00588623 & 0.01769641 \\
\hline
\end{tabular}


Table 3.4: Simulation settings for $\mathcal{C}$ for a 20-floor building. The meaning of the entries is as described in the caption of Table 3.1.

\begin{tabular}{c|cccc}
\hline Dist & Exp & InvExp & Gaussian & Bimodal \\
\hline$c_{1}$ & 0.59343035 & 0.00000002 & 0.00088438 & 0.00077597 \\
$c_{2}$ & 0.24127078 & 0.00000005 & 0.00240398 & 0.00306903 \\
$c_{3}$ & 0.09809338 & 0.00000013 & 0.00584751 & 0.00945329 \\
$c_{4}$ & 0.03988179 & 0.00000033 & 0.01272787 & 0.02267767 \\
$c_{5}$ & 0.01621473 & 0.00000081 & 0.02479051 & 0.04237177 \\
$c_{6}$ & 0.00659242 & 0.000002 & 0.04320761 & 0.06168651 \\
$c_{7}$ & 0.00268028 & 0.00000492 & 0.0673876 & 0.07013443 \\
$c_{8}$ & 0.00108972 & 0.00001211 & 0.09404697 & 0.06308409 \\
$c_{9}$ & 0.00044305 & 0.00002978 & 0.11745045 & 0.04806619 \\
$c_{10}$ & 0.00018013 & 0.00007324 & 0.13125312 & 0.04023324 \\
$c_{11}$ & 0.00007324 & 0.00018013 & 0.13125312 & 0.05156804 \\
$c_{12}$ & 0.00002978 & 0.00044305 & 0.11745045 & 0.0817498 \\
$c_{13}$ & 0.00001211 & 0.00108972 & 0.09404697 & 0.11525582 \\
$c_{14}$ & 0.00000492 & 0.00268028 & 0.0673876 & 0.12987546 \\
$c_{15}$ & 0.000002 & 0.00659242 & 0.04320761 & 0.11450328 \\
$c_{16}$ & 0.00000081 & 0.01621473 & 0.02479051 & 0.07868357 \\
$c_{17}$ & 0.00000033 & 0.03988179 & 0.01272787 & 0.04211504 \\
$c_{18}$ & 0.00000013 & 0.09809338 & 0.00584751 & 0.01755607 \\
$c_{19}$ & 0.00000005 & 0.24127078 & 0.00240398 & 0.00569962 \\
$c_{20}$ & 0.00000002 & 0.59343035 & 0.00088438 & 0.00144109 \\
\hline
\end{tabular}


information about the probability distributions of the calling floors, $\mathcal{C}$, to calculate the best possible parking floor. In both policies, we will use the average waiting time as the performance metric.

\subsubsection{Do_Nothing Policy: SEP1}

The Do_Nothing policy, referred to as SEP1, is formally presented in Algorithm 1. As we can observe from the algorithm, the simulation starts by selecting a random initial parking floor for the elevator. This is done in an equiprobable manner. We then simulate a number of passenger calls that require the elevator to go to the calling floor, dropping-off the passenger at the destination floor, and then parking the elevator car at the same destination floor while it waits for the next passenger call.

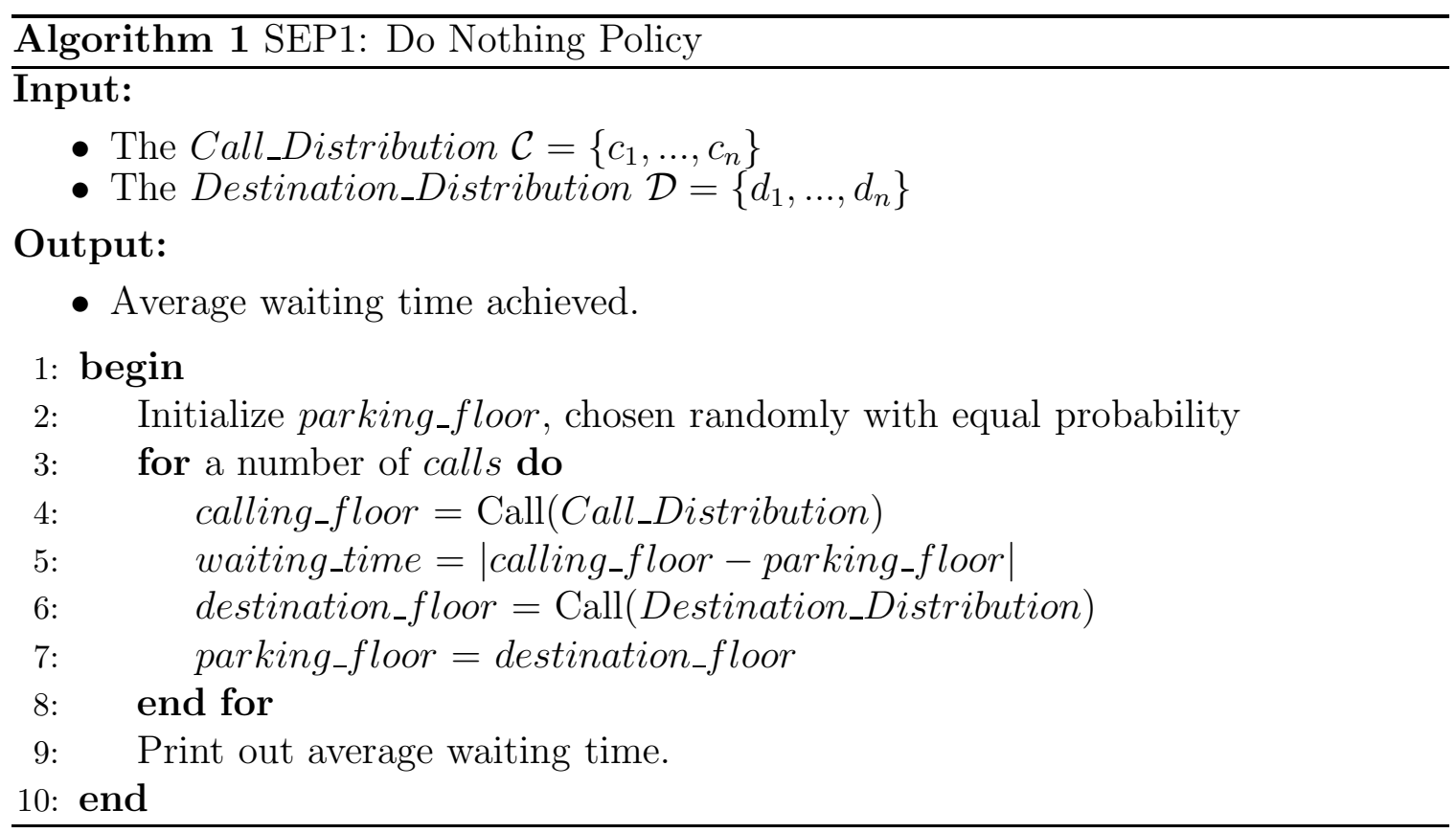

To evaluate the average waiting time, we calculated the waiting time for each call which, as mentioned, is used to evaluate the performance of the policy. This is done by using the following equation:

$$
W T=\theta * \mid \text { calling_floor }- \text { parking_floor } \mid,
$$


where $\theta$ is a parameter characterizing the pace variable, and which differs from one system to another because of different shaft speeds and the associated accelerations/decelerations of the elevator shafts. Since this is constant for all the simulations, we ignored this pace parameter and focused on the travel distance from the parked location to the floor where the call is made, |calling_floor - parking_floor $\mid$, which does not change for different elevator systems even if their paces are different. By doing this, the model would be generalized so that it can be applied to different elevator systems since it will be system independent. This yields the final waiting time equation to be:

$$
W T=\mid \text { calling_floor }- \text { parking_floor } \mid,
$$

which is used to calculate the waiting time for all the policies studied in the thesis.

\section{Simulation Results}

To test the models, we ran a number of simulations for different building settings, as mentioned in Section 3.3. We ran our tests on all the settings, but we will present and discuss, in the body of this chapter, the results for the 12-floor scenario with the four different types of call distributions specified above, and for a uniform destination distribution. A more detailed and comprehensive set of results and plots for the other types of buildings and distributions is included in Appendix A, but not reported here to enhance the readability.

In Table 3.5, we present the different call distributions for the 12-floor building, in which we tested SEP1 on, and the results of these simulations. The table shows the ensemble $A W T$ for passengers for an ensemble of 200 experiments, and where the number of iterations (i.e., passenger calls) was 1,000. In the table, Dist refers to the type of distribution used in that simulation, $c_{i}$ is the call probability at floor $i$, and $A W T$ is the corresponding average waiting time of the passengers in that experiment.

To cite one example, for the exponential distribution, Exp, we obtained an average waiting time, $A W T$, of 5.36. This means that if the building has call probabilities as per this distribution, the elevator with no intelligent parking policy will result in having an $A W T$ of almost 6 floors distance. 
Table 3.5: Simulation results for a 12-floor building for the policy SEP1 for an ensemble of 200 experiments. The results reported are the average waiting times for passengers in terms of number of floors for the elevator car to travel so as to reach the next call from the parked location.

\begin{tabular}{c|cccc}
\hline Dist & Exp & InvExp & Gaussian & Bimodal \\
\hline$c_{1}$ & 0.59344245 & 0.00002978 & 0.02592411 & 0.01270883 \\
$c_{2}$ & 0.24127569 & 0.00007324 & 0.04518336 & 0.03860891 \\
$c_{3}$ & 0.09809538 & 0.00018013 & 0.07046903 & 0.07525205 \\
$c_{4}$ & 0.0398826 & 0.00044306 & 0.09834746 & 0.09457958 \\
$c_{5}$ & 0.01621506 & 0.00108974 & 0.12282111 & 0.08017525 \\
$c_{6}$ & 0.00659255 & 0.00268033 & 0.13725493 & 0.06220755 \\
$c_{7}$ & 0.00268033 & 0.00659255 & 0.13725493 & 0.08440493 \\
$c_{8}$ & 0.00108974 & 0.01621506 & 0.12282111 & 0.14232761 \\
$c_{9}$ & 0.00044306 & 0.0398826 & 0.09834746 & 0.17475873 \\
$c_{10}$ & 0.00018013 & 0.09809538 & 0.07046903 & 0.13967666 \\
$c_{11}$ & 0.00007324 & 0.24127569 & 0.04518336 & 0.07169797 \\
$c_{12}$ & 0.00002978 & 0.59344245 & 0.02592411 & 0.02360195 \\
\hline$A W T$ & 5.36434 & 5.376775 & 3.60748 & 3.707325 \\
\hline
\end{tabular}


Similarly in the inverse exponential distribution, InvExp, SEP1 yielded very similar results, with an $A W T$ of 5.38 , which is very reasonable since it merely "inverses" the probabilities to be in an increasing order.

In the Gaussian distribution, Gaussian, the $A W T$ was reduced to 3.6 as the traffic was evenly more distributed across the building. Similarly, in the more complicated distribution, Bimodal, the value was close to the Gaussian, with an AWT of 3.7.

Since the parking policy in SEP1 depends on the destination floor, the $A W T$ is heavily affected by both $\mathcal{C}$ and $\mathcal{D}$. If both the distributions are skewed towards a specific area of the building, this will result in a small value for $A W T$. If, however, they are opposing each other, such as having $\mathcal{C}$ to be $\operatorname{Exp}$ and $\mathcal{D}$ to be InvExp, it will produce a very high $A W T$. These simulations results are included in Appendix A.

In Figure 3.2, we plot the results of the $A W T$ for an ensemble of 200 experiments for the Exp Distribution, where the number of passenger calls is 1,000. Observe that the SEP1 policy quickly leads to the final converged value of the $A W T$, in less than 50 passenger calls.

\subsubsection{Myopic Policy: SEP2}

The second policy, SEP2, was based on the model that was proposed in [22], referred to as a "Myopic Policy". The principle motivating it is that the model selects a predetermined floor that the elevator waits at for the next call. The model precomputes the best possible floor that the elevator should park at, so as to minimize the average waiting time. This model resorts to using the Call Distribution, $\mathcal{C}$, to determine that floor.

The main simulation follows the same process as the SEP1 policy in Section 3.4.1, where the elevator receives a call and then picks the passenger up at that floor and drops the passenger off at the destination. It then moves to the pre-determined floor to wait for the next call. The difference between SEP1 and SEP2 lies in the selection of the parking floor policy, where, instead of waiting at the drop-off floor, it moves to the pre-determined parking floor where it will wait for the next call. The 


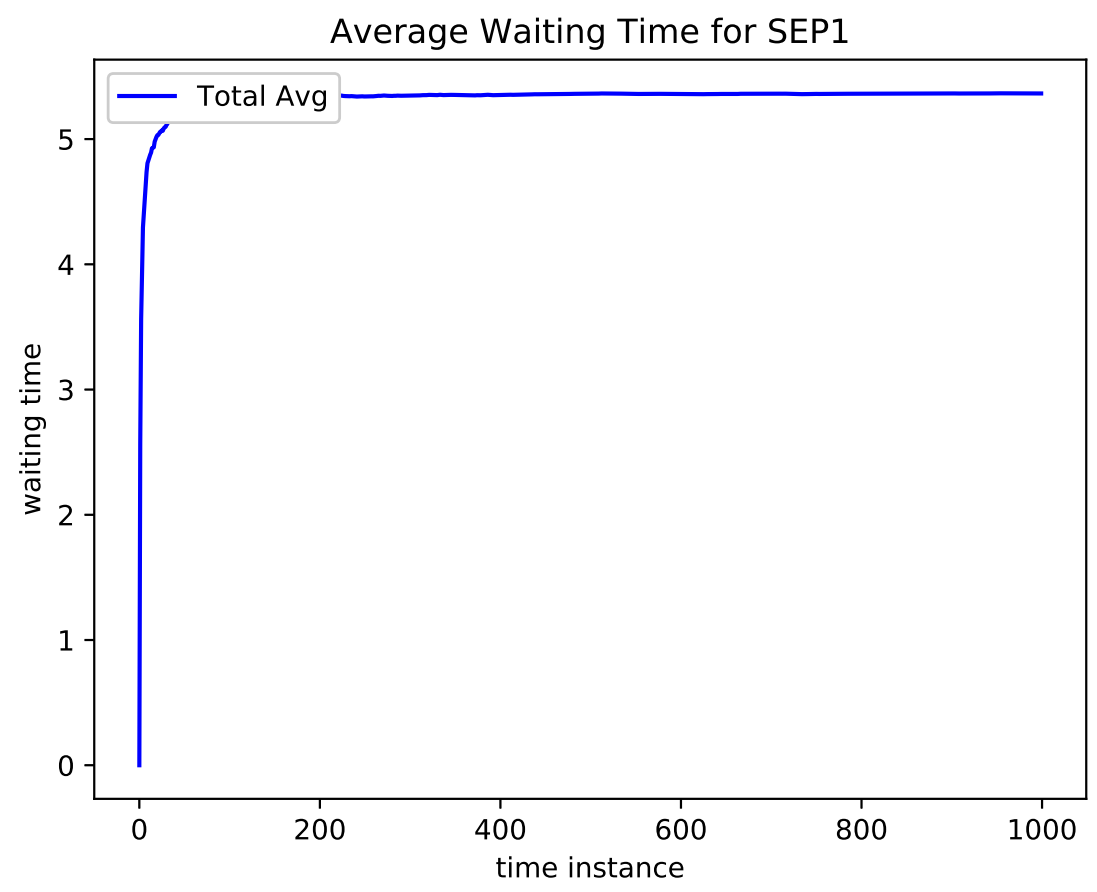

Figure 3.2: The Average Waiting Time for the "Do Nothing" Policy, SEP1, for an ensemble of 200 experiments for the case of the Exp distribution.

corresponding algorithm is in Algorithm 2.

Algorithm 3 shows the "optimal floor" selection algorithm that is used to compute the optimal parking floor. This is done by exhaustively searching across all the floors so as to compute which floor produces the minimum expected waiting time, as described in Equation (3.3).

$$
T(f)=\sum_{y=1}^{n}|y-f| * g(y),
$$

where $f$ is the floor selected as a parking floor, $n$ is the number of floors in the building, $g(y)$ is the probability of receiving a call from the floor $y$.

The main disadvantage of this policy is that it requires the a priori knowledge of $\mathcal{C}$ so as to calculate the expected waiting time for each floor. 


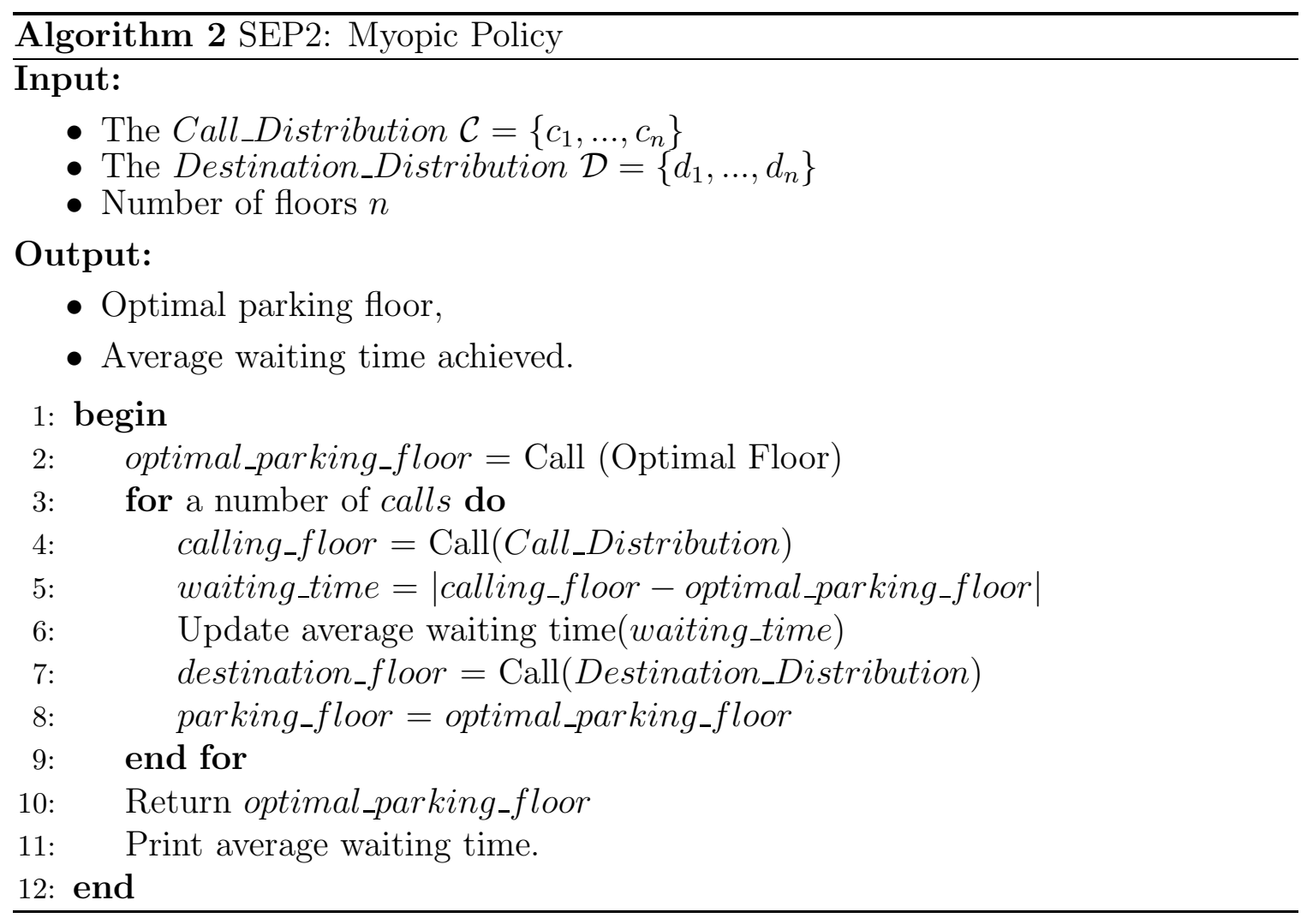




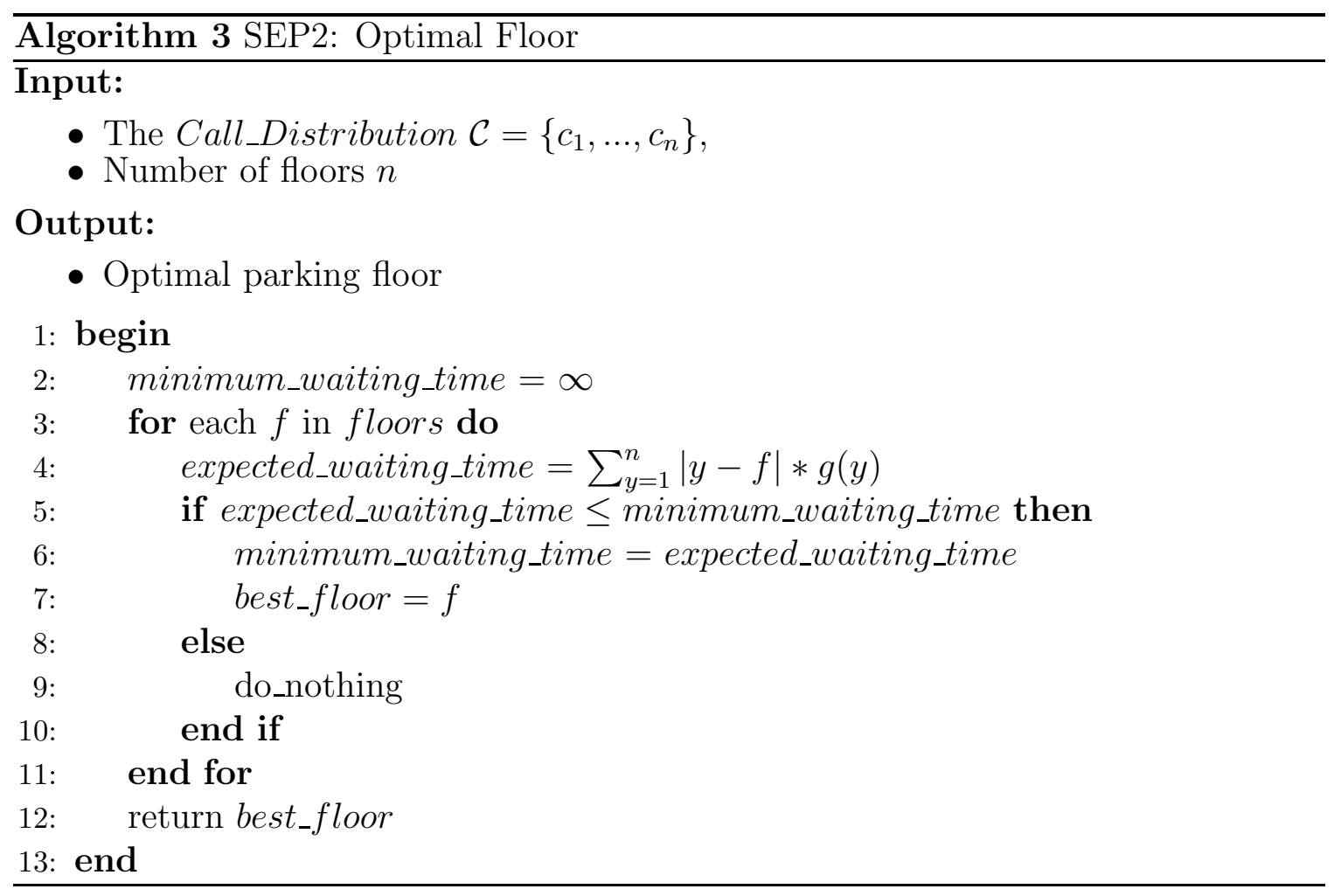

\section{Simulation Results}

To test the performance of SEP2, the myopic policy, we used the same building settings mentioned previously. For the sake of comparison, we will include the results for the same settings that we showed the results for, in SEP1. In Table 3.6, we use the same legend as in Table 3.5 with the addition of $B F$ which is the "best parking floor" calculated.

From Table 3.6, where we considered the case of 12 floors, we can see that the performance of SEP2 exceeded that of SEP1, especially when the distribution is skewed towards a specific floor, as in Exp and InvExp. The AWT in the Exp scenario decreased significantly from 5.36 to 0.71 . Unlike SEP1, the algorithm determined a floor that it will always wait at for the next call, which is the first floor in the Exp setting.

Similarly, in the InvExp scenario, the results showed a huge decrease in the $A W T$, and the algorithm determined that the best parking floor was floor 12 , and 
that resulted in an $A W T$ of 0.75 , which was reduced significantly from 5.38. Both the Exp and InvExp scenarios yielded a huge decrease in the AWT of about $85 \%$.

In the Gaussian and Bimodal cases, the improvements in the performance were not as significant as in the previous distributions, as most of the calls were more distributed across all the floors. So it is reasonable to expect a higher $A W T$ because more calls originated from other floors. For the Gaussian case, the algorithm picked floor 6 to be the optimal one, and that reduced the $A W T$ to 2.13 from 3.61. For the Bimodal case, floor 8 was selected to be optimal, and that reduced the AWT to 2.25 from 3.71. In both distributions, the decrease in waiting time was around $40 \%$.

Table 3.6: Simulation results for a 12-floor building for the policy SEP2 for an ensemble of 200 experiments. The results reported are the average waiting times for passengers in terms of number of floors for the elevator car to travel so as to reach the next call from the parked location.

\begin{tabular}{c|cccc}
\hline Dist & Exp & InvExp & Gaussian & Bimodal \\
\hline$c_{1}$ & 0.59344245 & 0.00002978 & 0.02592411 & 0.01270883 \\
$c_{2}$ & 0.24127569 & 0.00007324 & 0.04518336 & 0.03860891 \\
$c_{3}$ & 0.09809538 & 0.00018013 & 0.07046903 & 0.07525205 \\
$c_{4}$ & 0.0398826 & 0.00044306 & 0.09834746 & 0.09457958 \\
$c_{5}$ & 0.01621506 & 0.00108974 & 0.12282111 & 0.08017525 \\
$c_{6}$ & 0.00659255 & 0.00268033 & 0.13725493 & 0.06220755 \\
$c_{7}$ & 0.00268033 & 0.00659255 & 0.13725493 & 0.08440493 \\
$c_{8}$ & 0.00108974 & 0.01621506 & 0.12282111 & 0.14232761 \\
$c_{9}$ & 0.00044306 & 0.0398826 & 0.09834746 & 0.17475873 \\
$c_{10}$ & 0.00018013 & 0.09809538 & 0.07046903 & 0.13967666 \\
$c_{11}$ & 0.00007324 & 0.24127569 & 0.04518336 & 0.07169797 \\
$c_{12}$ & 0.00002978 & 0.59344245 & 0.02592411 & 0.02360195 \\
\hline BestFloor & 1 & 12 & 6 & 8 \\
\hline AWT & 0.71168 & 0.750515 & 2.131015 & 2.250805 \\
\hline
\end{tabular}

In Figure 3.3, we plot the results of the $A W T$ for an ensemble of 200 experiments for the Exp distribution, where the number of passenger calls is 1,000. Observe that for the SEP2, the value first increased steeply, and was then able to converge to a final small value of $A W T$ in less than 100 calls. This is very reasonable since it calculates 
the best floor before the simulation deals with the calls.

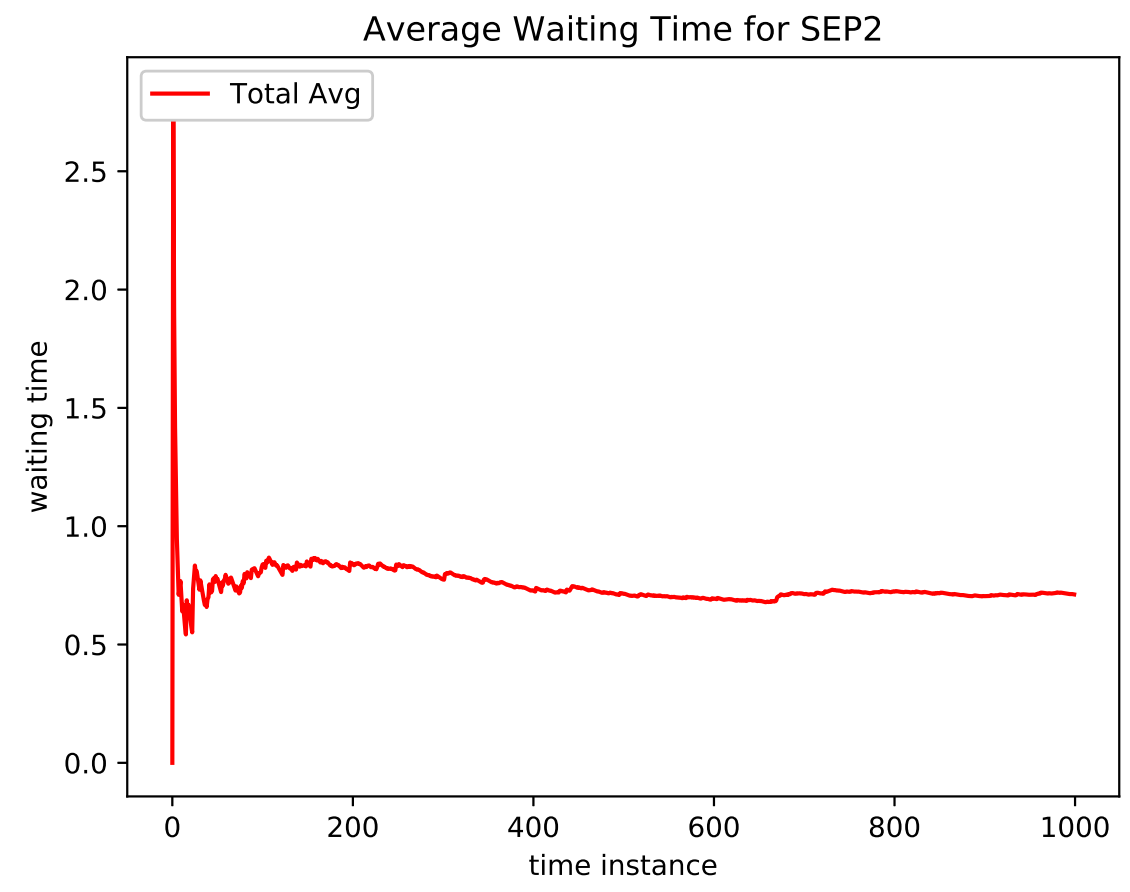

Figure 3.3: Average Waiting Time for the "Myopic Policy", SEP2, for an ensemble of 200 experiments for the case of Experiment Ex(1).

In Figure 3.4, one can observe the difference in the performance between the two policies, SEP1 and SEP2, and how the SEP2 outperformed SEP1 to produce a very low $A W T$ in comparison with SEP1.

The problem with SEP2 is that to achieve these results, the algorithm needed to know $\mathcal{C}$ so as to calculate the best parking floor. In the next section, we will show how one can obtain even more superior results or (close to the optimal results) with LA-based solutions that do not require this knowledge.

Additional results with tables and graphs for the other cases can be found in Appendix A. 


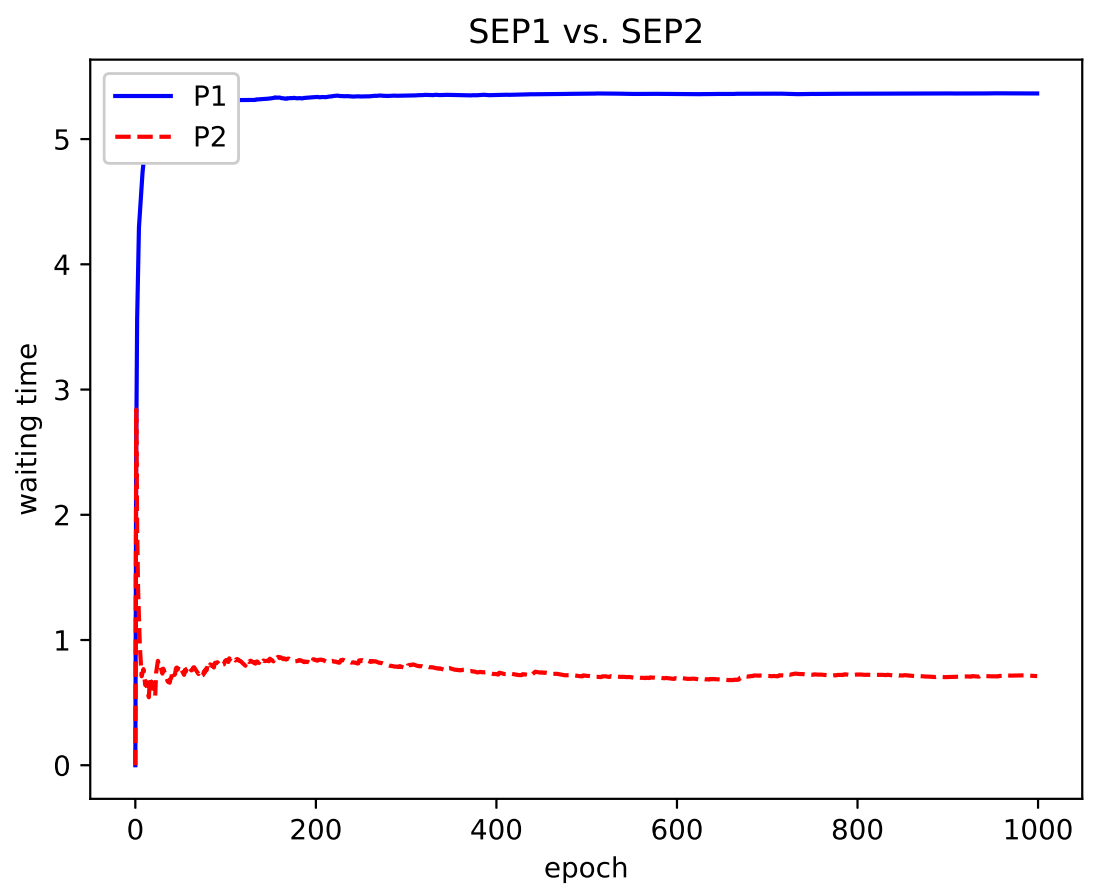

Figure 3.4: The Average Waiting Time for SEP1 vs. SEP2, for an ensemble of 200 experiments for the case of the Exp distribution.

\subsection{LA-Based Solutions}

In this section, we are going to present our proposed LA-based solutions for the SEP. First, we will show how we have modelled the problem, and thereafter we present an $L_{R I}$-based solution to the problem. Subsequently, we submit an enhancement on the $L_{R I}$ solution, in which we use the pursuit concept for the $L_{R I}$, and this yielded the second and even better solution, which is the $P L_{R I}$-based solution.

\subsubsection{Problem Modelling}

Before we present our proposed solutions, we need to explain how the problem was modelled so that it could be solved using an LA approach. As mentioned in Chapter 2, any LA structure consists of an Environment and the LA itself. The LA chooses one of the actions it is offered, i.e., one that is relevant to the problem domain, and then 
the Environment evaluates it and reacts based on the criterion it uses by responding with a reward or penalty feedback to the LA.

In the SEP, the modelling is much simpler than in the MEP investigated later. In the SEP, we modelled the floors as the actions of the LA, which, hopefully, will eventually converge to one that can be reckoned as the best parking floor. We then modelled the Environment so that it could provide us with the feedback about whether the selected floor was good or bad. In the former case the decision was rewarded, and in the latter, it was penalized.

To achieve this, we divided the SEP into two different parts, namely the controller and the evaluator. When it concerns the LA-based solution, we can say that the controller is the LA and the evaluator is the Environment, which evaluates the action selected by the controller or the LA.

The LA acts as the elevators' controller, which chooses one of the available floors or (actions) where the elevator will park at to wait for the next passenger call. On the other hand, the Environment evaluates the selected floor based on the objective or fitness function that is specified. One thing to note here is that the LA, or the controller, does not know anything about the distribution of passengers' calls, $\mathcal{C}$, unlike the solutions presented earlier.

\subsection{2 $\mathrm{L}_{R I}$-Based Solution: SEP3}

Our first proposed solution, referred to as SEP3, is based on the $L_{R I}$ scheme, where the LA updates the actions probabilities when it receives a reward from the Environment, and it does nothing when it receives a penalty. Based on the theory of LA, the $L_{R I}$ scheme helps us to achieve a near-optimal solution by updating the action probabilities to converge towards the best possible solution, which, in our case, is the best parking floor.

We present the corresponding algorithm in Algorithm 4. Initially, when the simulation of the experiments begins, the LA begins by selecting one of the available floors (actions) as the initial parking floor with equal probability and sends the selected floor to the Environment. Once the Environment receives the selected floor, 
it evaluates it based on the passengers' $A W T$ from the start of the simulation until that time instance, as shown in Algorithm 5. If the Environment evaluates that the waiting time is less than or equal to the $A W T$, it sends a reward feedback to the LA, informing it that it was a good choice. Otherwise, it sends a penalty feedback.

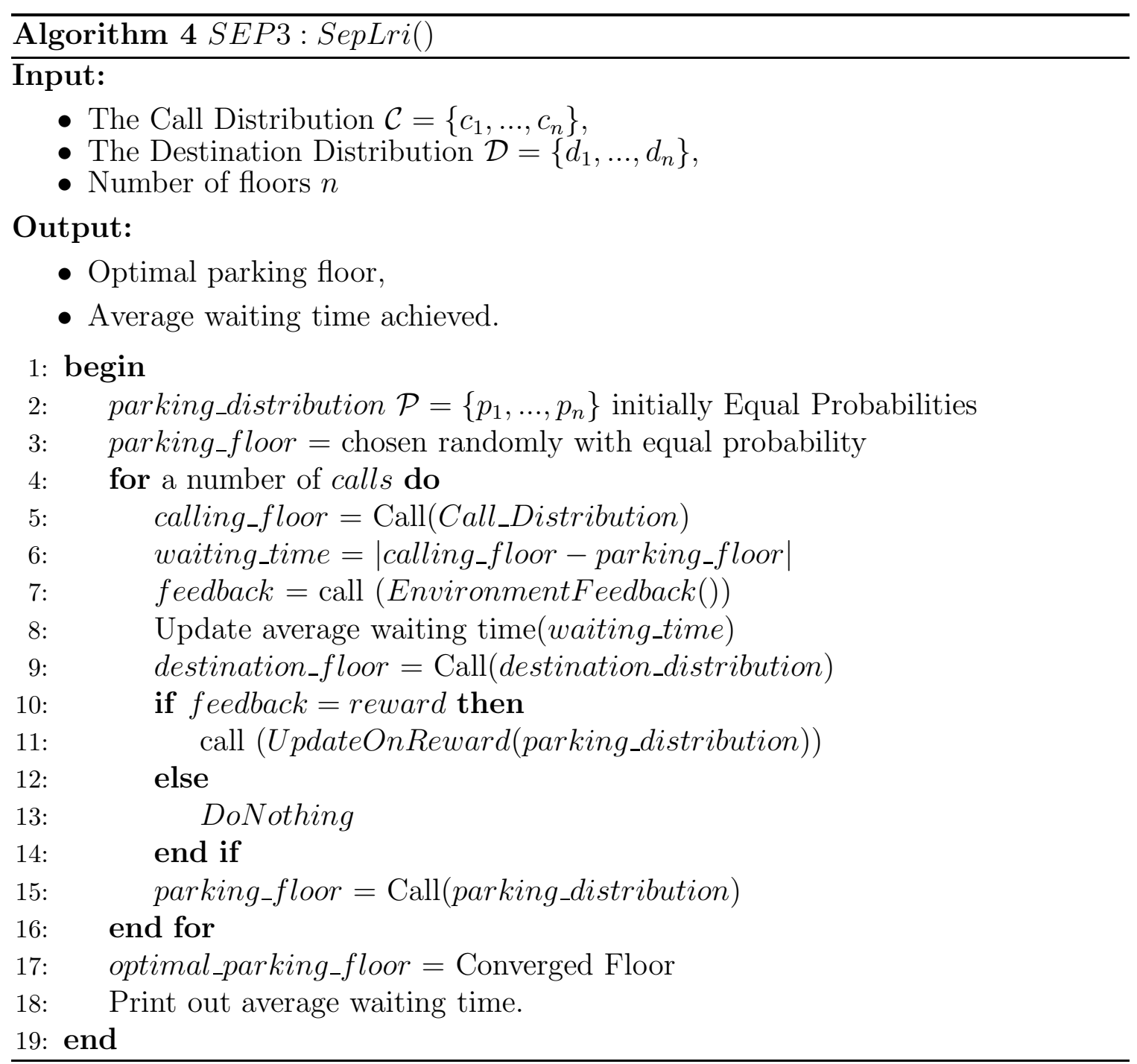

Once the LA receives the feedback, it checks whether it was a reward or a penalty. If it was a reward, the LA updates the probabilities of the floors according to the previously selected action in an $L_{R I}$ manner. In Algorithm 6 we present how the LA updates the probabilities of the floors. 
The LA then starts selecting a new parking floor based on the updated distribution, and repeats the process until it, hopefully, converges to the best parking floor.
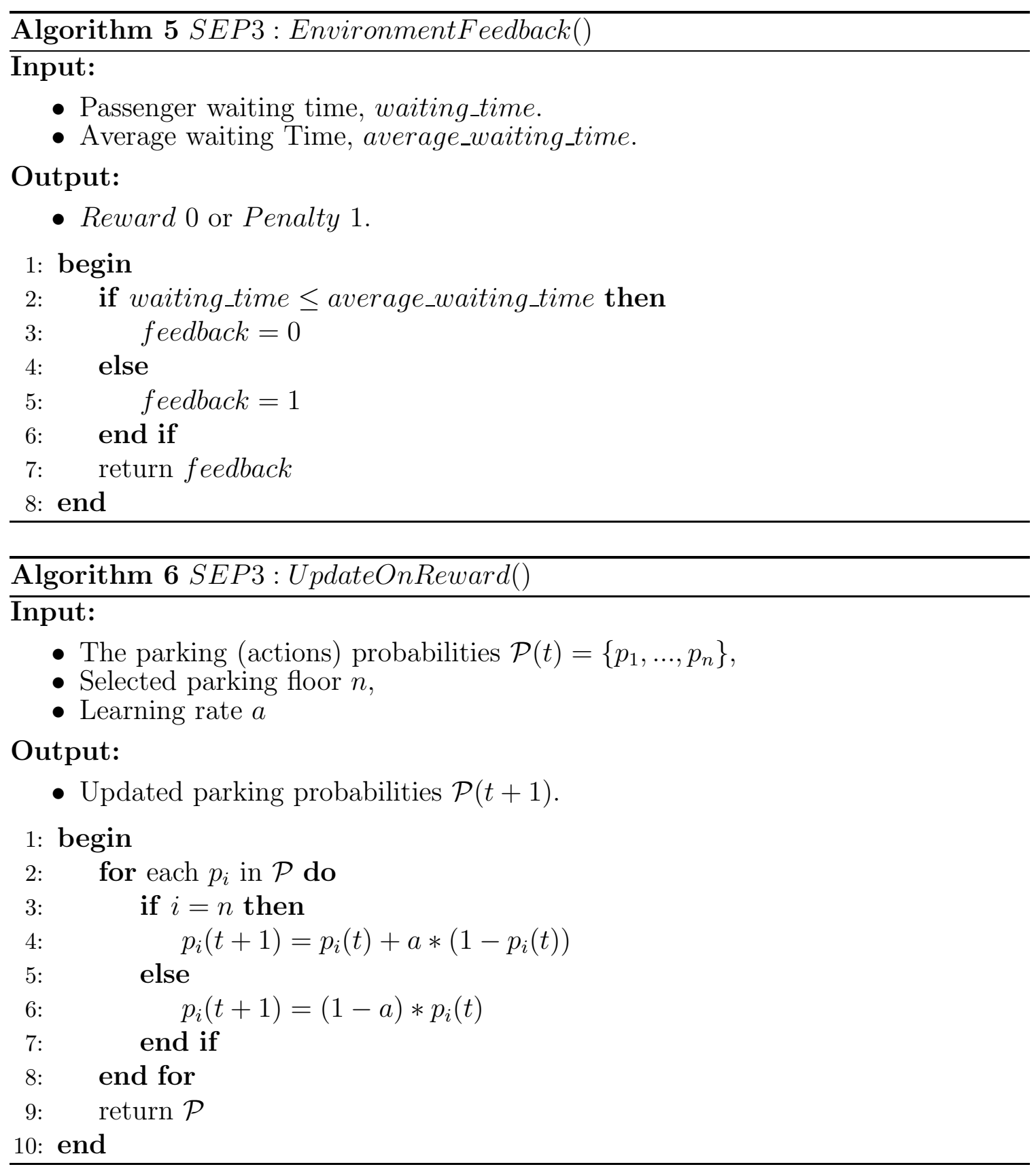


\section{Simulation Results}

To test our proposed solution, we used the same building settings that were used earlier in Sections 3.4.1 and 3.4.2 for the purpose of a fair comparison. We discuss the results below in Table 3.7.

From Table 3.7, one can observe that our proposed solution yielded the optimal parking floor with a value that is very close to the optimal $A W T$.

Consider the first scenario with the exponential distribution, Exp. The obtained results showed that the proposed solution converged to the optimal floor, which is the first floor, and was able to reach a better $A W T$ than SEP1 and very close to SEP2, with an $A W T$ of around 1.15 floors to reach the calling floor.

Similarly, in the second scenario, InvExp, our results demonstrated that the $L_{R I}$ algorithm was able to converge to a floor close to the best floor, which is the 11th floor. Since this distribution is merely the "inverse" of the previous distribution, this was a very reasonable choice. This yielded an $A W T$ of 1.19. Both the first and second scenarios showed a huge increase in performance in comparison with SEP1 and also produced results comparable to the SEP2 without requiring them to know $\mathcal{C}$ a priori.

In the third scenario, Gaussian, where most calls were not concentrated and where they were more evenly distributed, the results showed that it achieved a very low $A W T$ of 2.43 when it converged to floor 7 .

Similarly, for the final scenario, Bimodal, it was able to produce an even lower value of $A W T$ than the SEP1 with an $A W T$ of 2.56 , when it converged to floor 8 . One can observe here that the impact of the proposed algorithm is very noticeable in the first two scenarios where the calls were skewed towards a specific zone or a floor.

The overall performance of SEP3 yielded a significant decrease in the $A W T$ that was between $50 \%$ to almost $80 \%$ of SEP1's performance. It was also able to produce results that were very close to SEP2, where the optimal solution was known from the beginning. As opposed to this, in the LA-based case, we did not use any methods to calculate the best floor using any known distribution. Instead, SEP3 adapted to the Environment and was able to conclude the identity of the best floor to be used as a parking floor. 
Table 3.7: Simulation results for a 12-floor building for the policy SEP3 for an ensemble of 200 experiments. The results reported are the average waiting times for passengers in terms of number of floors for the elevator car to travel so as to reach the next call from the parked location.

\begin{tabular}{c|cccc}
\hline Dist & Exp & InvExp & Gaussian & Bimodal \\
\hline$c_{1}$ & 0.59344245 & 0.00002978 & 0.02592411 & 0.01270883 \\
$c_{2}$ & 0.24127569 & 0.00007324 & 0.04518336 & 0.03860891 \\
$c_{3}$ & 0.09809538 & 0.00018013 & 0.07046903 & 0.07525205 \\
$c_{4}$ & 0.0398826 & 0.00044306 & 0.09834746 & 0.09457958 \\
$c_{5}$ & 0.01621506 & 0.00108974 & 0.12282111 & 0.08017525 \\
$c_{6}$ & 0.00659255 & 0.00268033 & 0.13725493 & 0.06220755 \\
$c_{7}$ & 0.00268033 & 0.00659255 & 0.13725493 & 0.08440493 \\
$c_{8}$ & 0.00108974 & 0.01621506 & 0.12282111 & 0.14232761 \\
$c_{9}$ & 0.00044306 & 0.0398826 & 0.09834746 & 0.17475873 \\
$c_{10}$ & 0.00018013 & 0.09809538 & 0.07046903 & 0.13967666 \\
$c_{11}$ & 0.00007324 & 0.24127569 & 0.04518336 & 0.07169797 \\
$c_{12}$ & 0.00002978 & 0.59344245 & 0.02592411 & 0.02360195 \\
\hline BestFloor & 2 & 11 & 7 & 8 \\
\hline AWT & 1.14951 & 1.191435 & 2.434935 & 2.560355 \\
\hline
\end{tabular}


Figure 3.5 demonstrates the behaviour of the $A W T$ for SEP3 over the number of calls as it decreases to be close to a single floor.

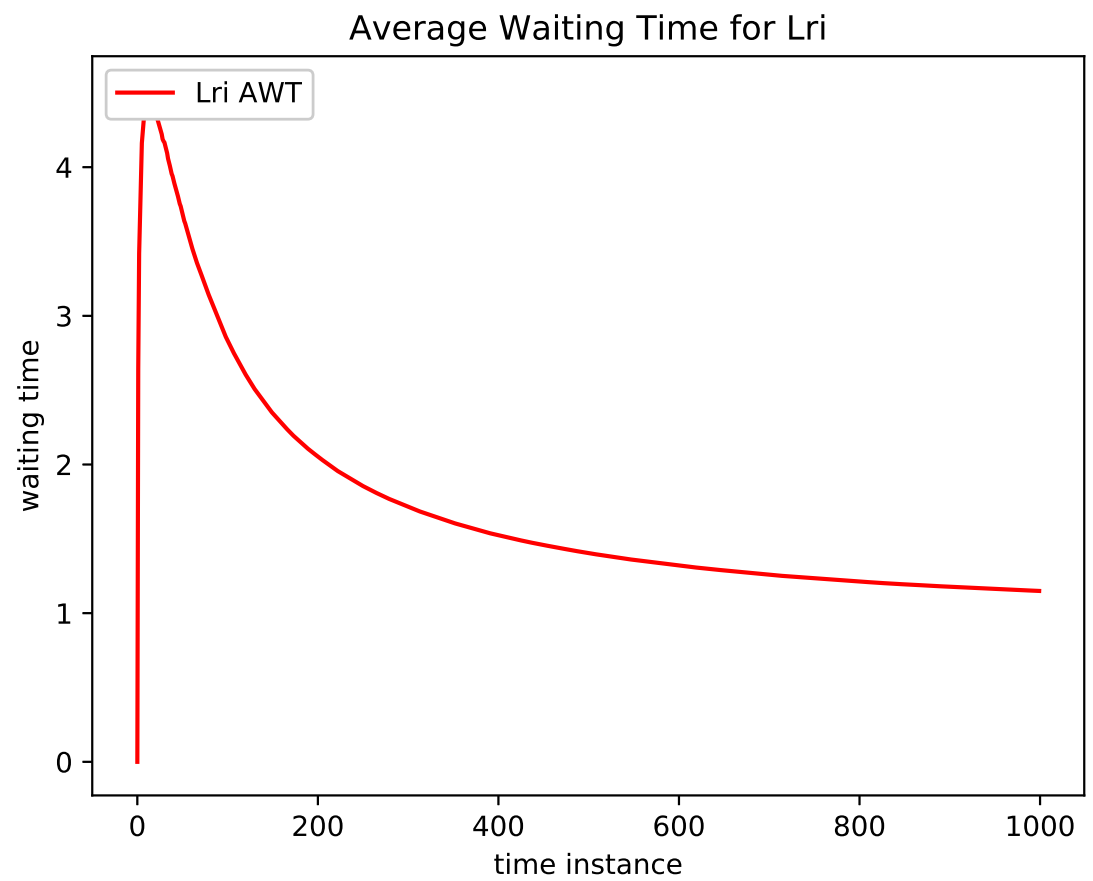

Figure 3.5: The Average Waiting Time for the $L_{R I}$ solution, SEP3, for an ensemble of 200 experiments for the case of the Exp distribution.

\subsection{3 $\mathrm{PL}_{R I^{-}}$Based Solution: SEP4}

The second LA-based solution, referred to as SEP4, is an improvement on the $L_{R I^{-}}$ based solution, SEP3, and it aimed to achieve an even better performance and faster convergence. To design it, we included the pursuit phenomenon described in Section 2.2.8. This allowed the LA to pursue the action with the superior reward ratio rather than just updating the selected action. The formal algorithm is shown in Algorithm 7.

From Algorithm 7, one can observe that it executes in the same manner as SEP3, but it differed when achieving the task of updating the action probabilities. For every action, it accomplished this by keeping track of the ratio of the rewards obtained to 
the number of times the action was selected. Consequently, we introduced the vector of reward estimates to keep track of the reward ratio.

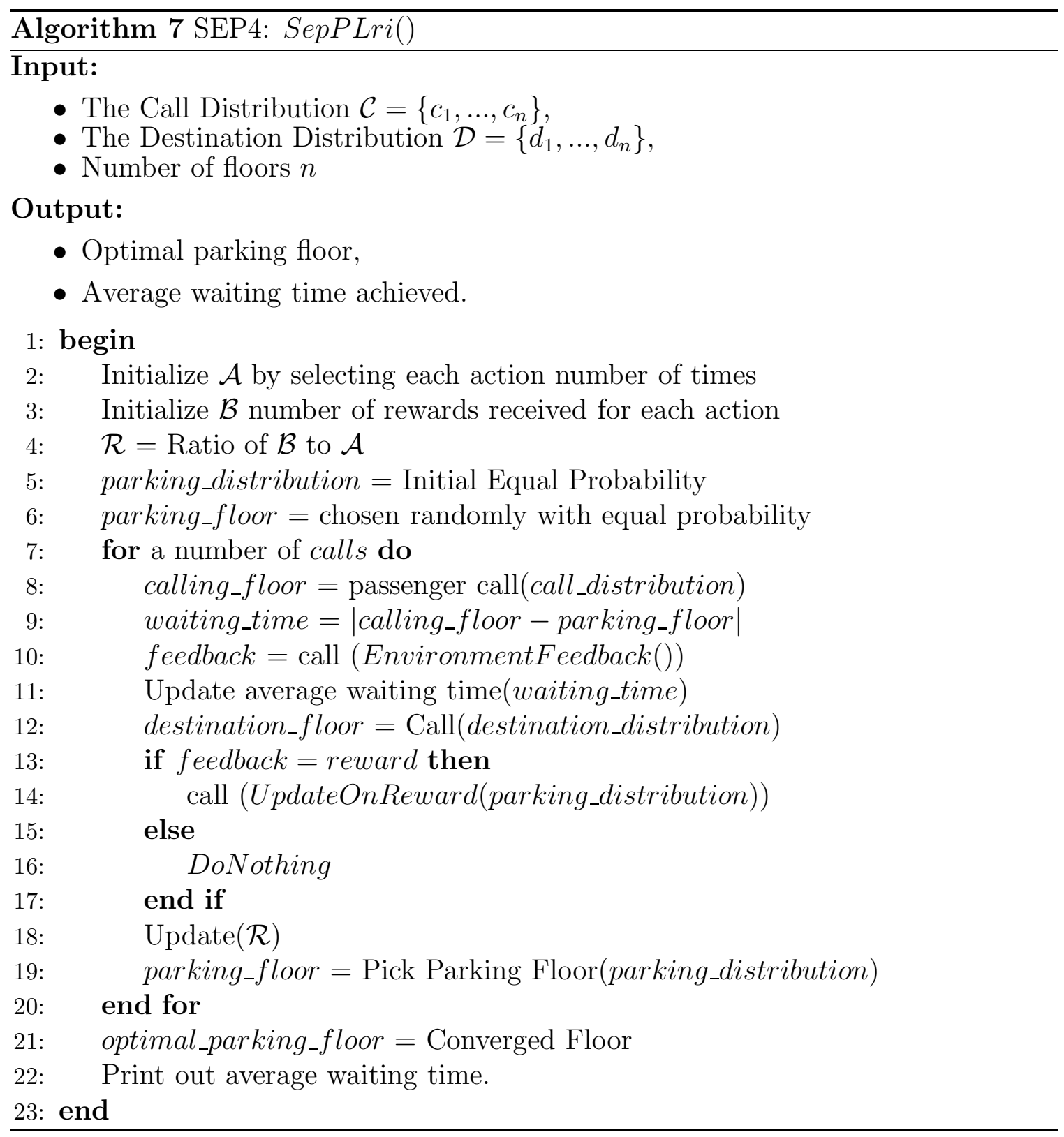

The simulation started by selecting each floor a small number of times (i.e., ten times each in our case), and recording the ratio of how many times each floor received a reward when compared to the number of times it was selected. Thereafter, the simulation proceeded along the same lines as the previous SEP3. The system first 
receives a call from a passenger, and it calculates the waiting time. It then requests the Environment for the feedback. The Environment evaluates the selected floor, and as done in Algorithm 5, it uses the average waiting time to decide on the feedback sent to the LA.

If the LA gets a reward, it updates the probabilities of the parking floors as in Algorithm 8. Instead of increasing the probability of selected floor, it pursues the one with the maximum reward estimate and increases its probability. This mechanism ensures that the algorithm converges towards the best solution faster than SEP3.

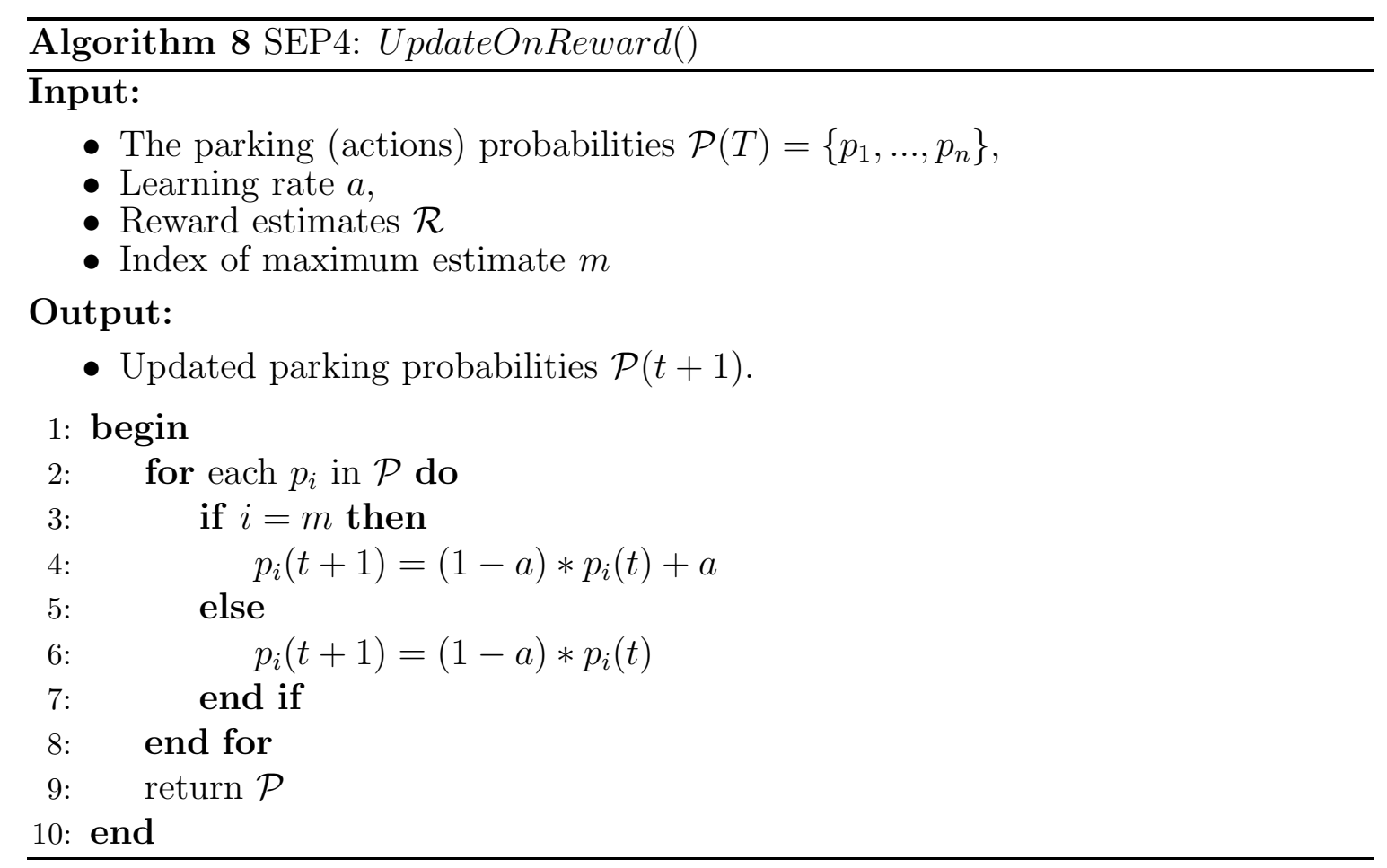

After that, the LA updates the rewards' estimates and again chooses a parking floor to be evaluated, and repeats the same cycle until it converges.

\section{Simulation Results}

To test SEP4, we used the same building settings as the previous solutions, SEP1, SEP2 and SEP3. Table 3.8 presents the results acquired from the simulations of the four distributions, and the corresponding results for SEP4. 
Table 3.8: Simulation results for a 12-floor building for the policy SEP4 for an ensemble of 200 experiments. The results reported are the average waiting times for passengers in terms of number of floors for the elevator car to travel so as to reach the next call from the parked location.

\begin{tabular}{c|cccc}
\hline Dist & Exp & InvExp & Gaussian & Bimodal \\
\hline$c_{1}$ & 0.59344245 & 0.00002978 & 0.02592411 & 0.01270883 \\
$c_{2}$ & 0.24127569 & 0.00007324 & 0.04518336 & 0.03860891 \\
$c_{3}$ & 0.09809538 & 0.00018013 & 0.07046903 & 0.07525205 \\
$c_{4}$ & 0.0398826 & 0.00044306 & 0.09834746 & 0.09457958 \\
$c_{5}$ & 0.01621506 & 0.00108974 & 0.12282111 & 0.08017525 \\
$c_{6}$ & 0.00659255 & 0.00268033 & 0.13725493 & 0.06220755 \\
$c_{7}$ & 0.00268033 & 0.00659255 & 0.13725493 & 0.08440493 \\
$c_{8}$ & 0.00108974 & 0.01621506 & 0.12282111 & 0.14232761 \\
$c_{9}$ & 0.00044306 & 0.0398826 & 0.09834746 & 0.17475873 \\
$c_{10}$ & 0.00018013 & 0.09809538 & 0.07046903 & 0.13967666 \\
$c_{11}$ & 0.00007324 & 0.24127569 & 0.04518336 & 0.07169797 \\
$c_{12}$ & 0.00002978 & 0.59344245 & 0.02592411 & 0.02360195 \\
\hline BestFloor & 1 & 12 & 6 & 8 \\
\hline AWT & 0.84734 & 0.839 & 2.36355 & 2.53346 \\
\hline
\end{tabular}


In the first scenario, Exp, the results acquired showed an improvement in the performance with comparison to SEP3. The $A W T$ decreased even more as a result of a faster convergence to the optimal location and even closer results to the optimal values of SEP2 with an AWT of 0.85 down from 1.15.

Similarly, in the second scenario, InvExp, SEP4 displayed a superior performance with a similar decrease in $A W T$ from 1.19 to 0.84 . We can see the significant impact on the performance, and how the faster convergence led to almost a $30 \%$ decrease in the $A W T$ when compared to SEP3.

In the third scenario, Gaussian, the results in SEP4 showed better results than SEP3, but the increase in the performance was not as significant as the previous scenarios with a decrease in the $A W T$ from 2.43 to 2.37 .

Similarly, for the final scenario, Bimodal, it was able to produce a lower $A W T$ than SEP3 with an $A W T$ of 2.53 down from 2.56 and converged to floor 8 . One can observe how the effect of the pursuit concept in SEP4 helps to increase the performance and produce superior results in all scenarios, and how this improvement is affected by $\mathcal{C}$.

Figure 3.6 displays the behaviour of how the $A W T$ decreased over time for the first scenario, Exp for SEP4.

In Figure 3.7, we can see the difference in convergence speed between the SEP3 and SEP4, where the SEP4 can be observed to display a better performance and faster convergence, which can both be attributed to incorporating the pursuit concept.

\subsection{Discussion}

We now comparatively discuss the simulations results obtained from the previous solutions. In Table 3.9, we submit all the results of the four solutions that were discussed, namely, SEP1, SEP2, SEP3 and SEP4. From the table, we see that SEP1, which we believe is the most popular policy currently used in buildings, performed very poorly in comparison to our proposed solutions. The improvement in the average waiting time was more than $50 \%$ and up to $80 \%$. This policy serves as a lower bound 


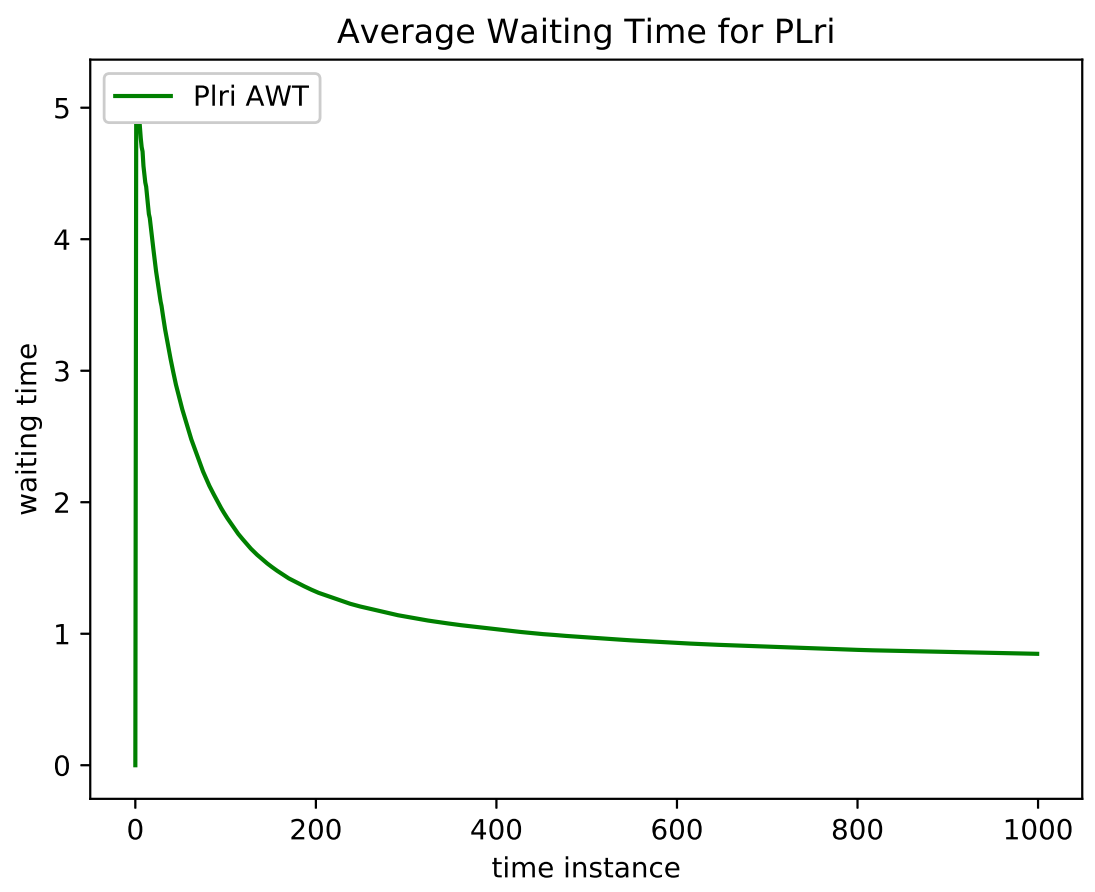

Figure 3.6: The Average Waiting Time for the $P L_{R I}$ solution, SEP4, for an ensemble of 200 experiments for the case of the Exp distribution.

for our benchmark, as no solution should be worse than this.

Table 3.9: Simulation results for the previous and newly proposed solutions for the SEP for an ensemble of 200 experiments for the 12 -floor settings. The results are given here as a tuple $(\alpha, \beta)$ where the first field, $\alpha$, is the best optimal parking floor and the second field, $\beta$ is the $A W T$ in terms of number of floors travelled for the elevator to reach the passenger from the parked location.

\begin{tabular}{|c|c|c|c|c|}
\hline Dist & SEP1 & SEP2 & SEP3 & SEP4 \\
\hline $\operatorname{Exp}$ & $(-, 5.364)$ & $(1,0.712)$ & $(2,1.150)$ & $(1,0.847)$ \\
\hline InvExp & $(-, 5.377)$ & $(12,0.751)$ & $(11,1.191)$ & $(12,0.839)$ \\
\hline Gaussian & $(-, 3.607)$ & $(6,2.131)$ & $(7,2.435)$ & $(6,2.364)$ \\
\hline Bimodal & $(-, 3.707)$ & $(8,2.251)$ & $(8,2.560)$ & $(8,2.533)$ \\
\hline
\end{tabular}

SEP2 was able to give us the optimal parking floors from the beginning, but it required the a priori knowledge of $\mathcal{C}$ for each floor. On the other hand, our LA-based 


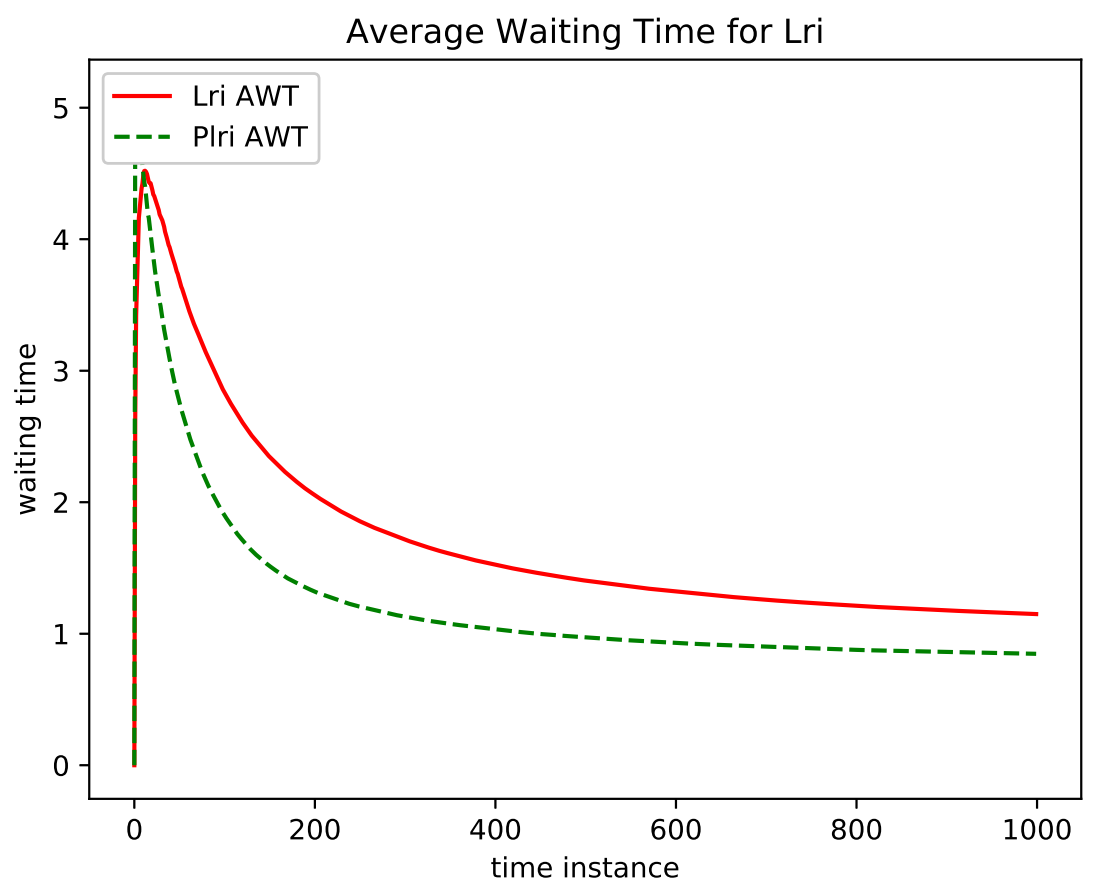

Figure 3.7: The Average Waiting Time for the $L_{R I}$ solution, SEP3, vs. $P L_{R I}$ solution, SEP4, for an ensemble of 200 experiments for the case of the Exp distribution.

solutions were able to achieve a close-to-optimal $A W T$ without the knowledge of $\mathcal{C}$.

SEP3 showed that it was better than SEP1 and recorded an $A W T$ improvement in Exp of $78.56 \%$, InveExp of $77.85 \%$, Gaussian of $32.49 \%$ and Bimodal of $30.9 \%$. Moreover, the results were close to the values achieved by SEP2.

We attempted to improve SEP3 by proposing SEP4 that incorporated the Pursuit concept. Here, we achieved even better results than obtained for the SEP3. The algorithm helped the system to converge faster to the optimal locations. It also resulted in a better overall $A W T$. The improvements were $26.3 \%$ for the Exp, $29.5 \%$ for the InvExp, 3\% for the Gaussian and 1\% for the Bimodal distributions.

One result that we found is that the more equally distributed the calls are, for example for the Gaussian distribution, the higher the $A W T$ will be. On the other hand, the importance of having a good policy shines when the calling distribution is skewed toward a specific region or a specific floor. Also, one can observe how the 
policies affected the $A W T$ in the first and the second scenarios, Exp and InvExp.

In Figure 3.8, we present the performance of each algorithm and how our proposed LA-based algorithms were able to achieve an average waiting time that is very close to the optimal solution, SEP2, and how it significantly outperforms SEP1.

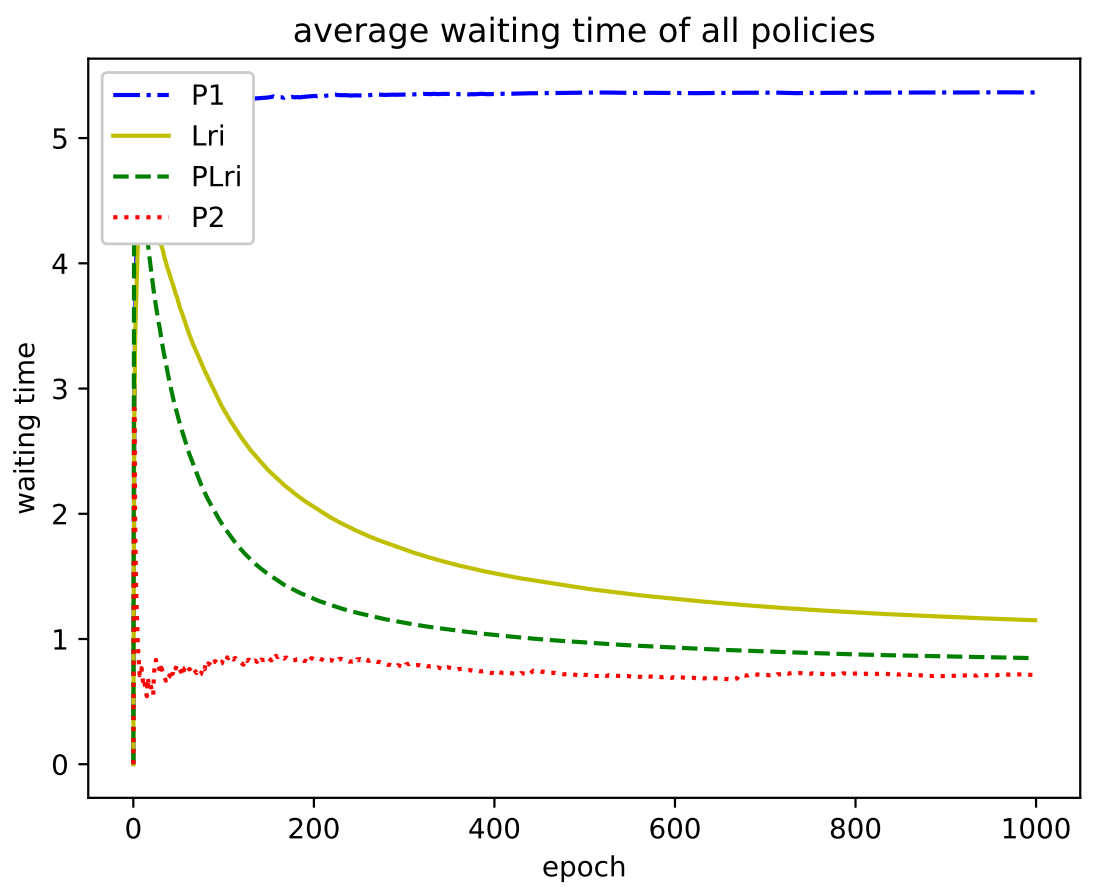

Figure 3.8: The Average Waiting Time for SEP1, SEP2, SEP3 and SEP4 for an ensemble of 200 experiments for the case of the Exp distribution, given in the graph as $P 1, P 2, L_{R I}, P L_{R I}$ respectively.

As mentioned earlier, additional results for all settings are given in Appendix A.

\subsection{Conclusion}

In this chapter, we reviewed different parking policies that were used in various building settings and for previously-reported solutions. We discussed two different solutions in the single elevator scenario and tabulated the experimental results that correspond to the various simulations. We then introduced our $L_{R I}$-based solution, SEP3, and it achieved the optimal parking floor without knowing $\mathcal{C}$ a priori. 
We further introduced an improvement on SEP3 to incorporate the Pursuit concept. This yielded our second solution, SEP4, a $P L_{R I}$-based solution. From the simulation results for SEP4, we demonstrated that it performed even better than SEP3.

This chapter included our findings and compared the results of our proposed solutions to the previous solutions. We showed that our LA-based solutions performed better than SEP1 and converged to the optimal floor. They also reduced the AWT to be close to the optimal value with the advantage that they did not require us to know the distributions to determine the best floor.

In the next chapter, we will discuss the more complicated scenario, namely one which deals with more than a single elevator shaft, the MEP. 


\section{Chapter 4}

\section{Solutions for the MEP}

\subsection{Introduction}

In this chapter, we will tackle the more complicated scenario which is the MEP, in which the building has more than a single elevator. We will initially submit the solutions used as benchmarks and show their results. After that, we will introduce our LA-based solutions and display their simulation results. We will then compare our findings with the benchmark results to demonstrate that we have obtained similar or better results.

In the literature, many papers have tackled the MEP from the perspective of the dispatching problem. Few, however, have tackled the problem of determining the optimal floors for the elevators, so as to reduce the average waiting time. Thus, we consider two scenarios to be used as benchmarks. The first one, referred to as MEP1, is to park the elevator at the current destination. We believe that this policy is the

most-widely used one in modern day buildings. The second policy is an extension of the previously-mentioned SEP2, where we predetermine a specific floor as an optimal parking floor, and the elevator always goes to that floor after each drop-off. However, in the case of the MEP, the optimal locations will be a set of floors that the elevators will park at. 


\subsection{Models of Computation}

As mentioned earlier in Section 3.2, there were two models of computations that were taken into consideration when solving the SEP. In the case of the MEP, we will use the same models. However, we will extend them to cover the multi-elevator scenario. The main competitive solution will require that the pick-up and drop-off distributions are known a priori, and we will use them to calculate the optimal parking floors for the MEP setting.

On the other hand, the other model is when the distributions are unknown to the controller or the decision-making algorithm. In this case, the MEP is solved using LA-based solutions. As we used LA for the SEP, we will show that a similar but extended version of the general LA-based solutions, can be applied to the MEP.

The formalization is as mentioned in Section 3.2 where there are two distributions $\mathcal{C}$ and $\mathcal{D} . \mathcal{C}=\left\{c_{1}, \ldots, c_{n}\right\}$ specifies the probabilities of passengers requesting to be picked up, where $c_{i}$ is the probability that a passenger requests a pickup from floor $i$. Similarly, $\mathcal{D}=\left\{d_{1}, \ldots, d_{n}\right\}$, where $d_{j}$ specifies the probability that a passenger will alight at floor $j$. Our computation model assumes that $\sum_{i=1}^{n} c_{i}=1$ and $\sum_{j=1}^{n} d_{j}=1$. Throughout our study, we will assume that all elevators process a passenger's request after it comes to rest. The dispatching criterion is outside the scope of this thesis, as we are concerned about the optimal parking locations only during the idle period. We will also assume that the car can only pick one passenger at a time and that all previous requests have been served.

\subsection{Simulation Settings}

Throughout our simulations, we incorporated different settings so as to test our models on different scenarios, and these settings were tested across all the proposed solutions for comparison purposes. The settings differed from the SEP case because we introduced the addition of extra elevator shafts. To calculate the number of required elevators, we assumed that each building would require elevators depending on their height, and we used one additional elevator for every 7 floors. Hence, we used the 
following equation to determine how many elevators we needed:

$$
E=\left\lceil\frac{\text { floors }}{7}\right\rceil .
$$

For our simulations, we used four different building settings. Just as in the SEP case, we tested our models on 8-floor, 12-floor, 16-floor and 18-floor buildings with 2, 2, 3 and 3 elevators respectively. For each case we used four different distributions for $\mathcal{C}$, which were as introduced in Section 3.3:

1. The first is an exponential distribution, referred to as Exp, designed to simulate an up-peak traffic pattern, where most calls come from the ground and/or lower floors;

2. The second distribution is the inverse exponential distribution, referred to as InvExp, that represents a down-peak traffic pattern, where most calls are from the upper floors;

3. The third distribution is the Gaussian (Normal) distribution, referred to as Gaussian, and this represents the traffic of passengers during the middle of the day, also referred to as "regular traffic";

4. The final distribution that we will use is a bimodal distribution, referred to as Bimodal, which is specified as a mixture of Gaussian distributions to represent a more complex "regular traffic" pattern.

The corresponding probabilities for the distributions mentioned above are represented in Section 3.3 in Tables 3.1, 3.2, 3.3 and 3.4 for 8, 12, 16 and 20-floor buildings respectively.

\subsection{Previous Solutions}

We first introduce the two models that have been considered as benchmarks for the MEP. The first policy is the one that we believe is the most dominant policy used in modern-day buildings, and the principle motivating it is that when one of the elevators 
drops a passenger off at the desired destination, it parks at that floor waiting for the next call. The second policy used as a benchmark is the extension of the one studied previously in [22] (used earlier in Section 3.4.1) to be applied to the MEP case. It is based on calculating the best parking floors as per the call distribution, $\mathcal{C}$.

\subsubsection{Do Nothing Policy: MEP1}

The first policy, called the "Do Nothing" policy, is referred to as MEP1, and is presented in Algorithm 9. As one can observe, the simulations start by having the elevators parked at initial random floors, selected in an equiprobable manner. Thereafter, a call is issued according to the distribution $\mathcal{C}$. The main controller then checks which elevator is the closest to the calling floor and sends that elevator to pick up the passenger. Once the elevator drops off the passenger at the destination floor, which is determined according to the distribution $\mathcal{D}$, the elevator waits at that drop-off floor and stops for the next call.

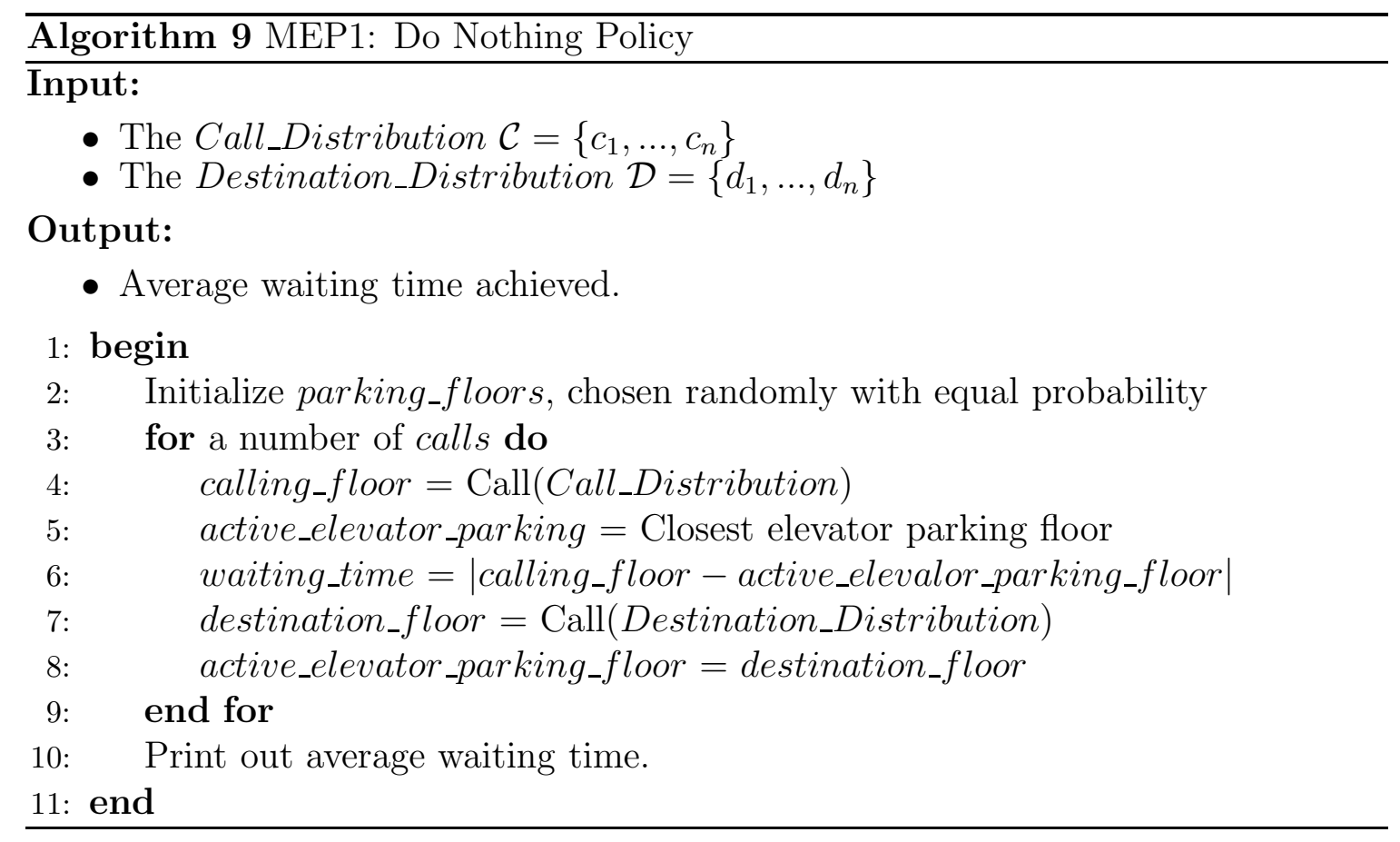

To evaluate the performance of the policy, we used the same metric mentioned in Equation (3.2), which is the average waiting time for the passengers. 


\section{Simulation Results}

To test the models in this chapter, we used the building settings mentioned in Section 4.3. We ran the tests and the simulations across all building settings, but in the body of this chapter, we will submit and discuss only a single setting of the building, which is the 12-floor building with 2 elevator shafts. More comprehensive results are presented in Appendix B.

In Table 4.1, we present the different call distributions for the 12-floor building, in which we tested MEP1 on, and the results of these simulations. The table shows the ensemble $A W T$ for passengers for an ensemble of 200 experiments, and where the number of iterations (i.e., passenger calls) was 1,000. In the table, Dist refers to the type of distribution used in that simulation, $c_{i}$ is the call probability at floor $i$, and $A W T$ is the corresponding average waiting time of the passengers in that experiment.

Table 4.1: Simulation results for a 12-floor building for the policy MEP1 for an ensemble of 200 experiments. The results reported are the average waiting times for passengers in terms of number of floors for the active elevator cars to travel so as to reach the next call from the parked location.

\begin{tabular}{c|cccc}
\hline Dist & Exp & InvExp & Gaussian & Bimodal \\
\hline$c_{1}$ & 0.59344245 & 0.00002978 & 0.02592411 & 0.01270883 \\
$c_{2}$ & 0.24127569 & 0.00007324 & 0.04518336 & 0.03860891 \\
$c_{3}$ & 0.09809538 & 0.00018013 & 0.07046903 & 0.07525205 \\
$c_{4}$ & 0.0398826 & 0.00044306 & 0.09834746 & 0.09457958 \\
$c_{5}$ & 0.01621506 & 0.00108974 & 0.12282111 & 0.08017525 \\
$c_{6}$ & 0.00659255 & 0.00268033 & 0.13725493 & 0.06220755 \\
$c_{7}$ & 0.00268033 & 0.00659255 & 0.13725493 & 0.08440493 \\
$c_{8}$ & 0.00108974 & 0.01621506 & 0.12282111 & 0.14232761 \\
$c_{9}$ & 0.00044306 & 0.0398826 & 0.09834746 & 0.17475873 \\
$c_{10}$ & 0.00018013 & 0.09809538 & 0.07046903 & 0.13967666 \\
$c_{11}$ & 0.00007324 & 0.24127569 & 0.04518336 & 0.07169797 \\
$c_{12}$ & 0.00002978 & 0.59344245 & 0.02592411 & 0.02360195 \\
\hline$A W T$ & 5.3604 & 5.3697 & 2.6252 & 2.6770 \\
\hline
\end{tabular}

One can observe that in Table 4.1, for the exponential distribution, Exp, we obtained an average waiting time, $A W T$, of 5.36. This means that if the building has 
call probabilities as per this distribution, the multiple elevators with no intelligent parking policy would result in having an $A W T$ of almost 6 floors' distance. This result is quite similar to the one obtained from the previous scenario where we had only a single elevator.

Similarly, in the inverse exponential distribution, InvExp, MEP1 yielded very similar results, with an $A W T$ of 5.37 , which is again very reasonable since it is the "inverse" of the probabilities in an increasing order.

For the Gaussian distribution, Gaussian, the $A W T$ was reduced to 2.65 as the traffic was more evenly distributed across the building. Similarly, in the more complicated distribution, Bimodal, the value was close to the Gaussian, with an $A W T$ of 2.68 .

Just as in the case of SEP1, MEP1 depends on the destination floor, and the $A W T$ is significantly affected by both $\mathcal{C}$ and $\mathcal{D}$. If both the distributions are skewed towards a specific area of the building, this will result in a small value for $A W T$. If, however, they are opposing each other, such as having $\mathcal{C}$ to be $\operatorname{Exp}$ and $\mathcal{D}$ to be InvExp, it will produce a very high $A W T$.

In Figure 4.1, we plot the results of the $A W T$ for an ensemble of 200 experiments for the Exp Distribution, where the number of passenger calls was 1,000. Observe that the MEP1 policy quickly leads to the final converged value of the $A W T$, in less than 300 passenger calls.

\subsubsection{Myopic Policy: MEP2}

The second policy, MEP2, is an extension to the previous solution for the single elevator scenario, SEP2, but modified to work with the multi-elevator setting, as explained in [22]. The model uses the principle of using the call distributions, $\mathcal{C}$, to calculate the best possible parking floors for the number of available elevators.

The simulation followed the same procedure as in the previously presented model, MEP1, where the elevators received a passenger call and then decided which elevator was to serve this passenger based on how far the elevators were, and where the closest one was, which was the one chosen to serve the current passenger. Subsequently, the 


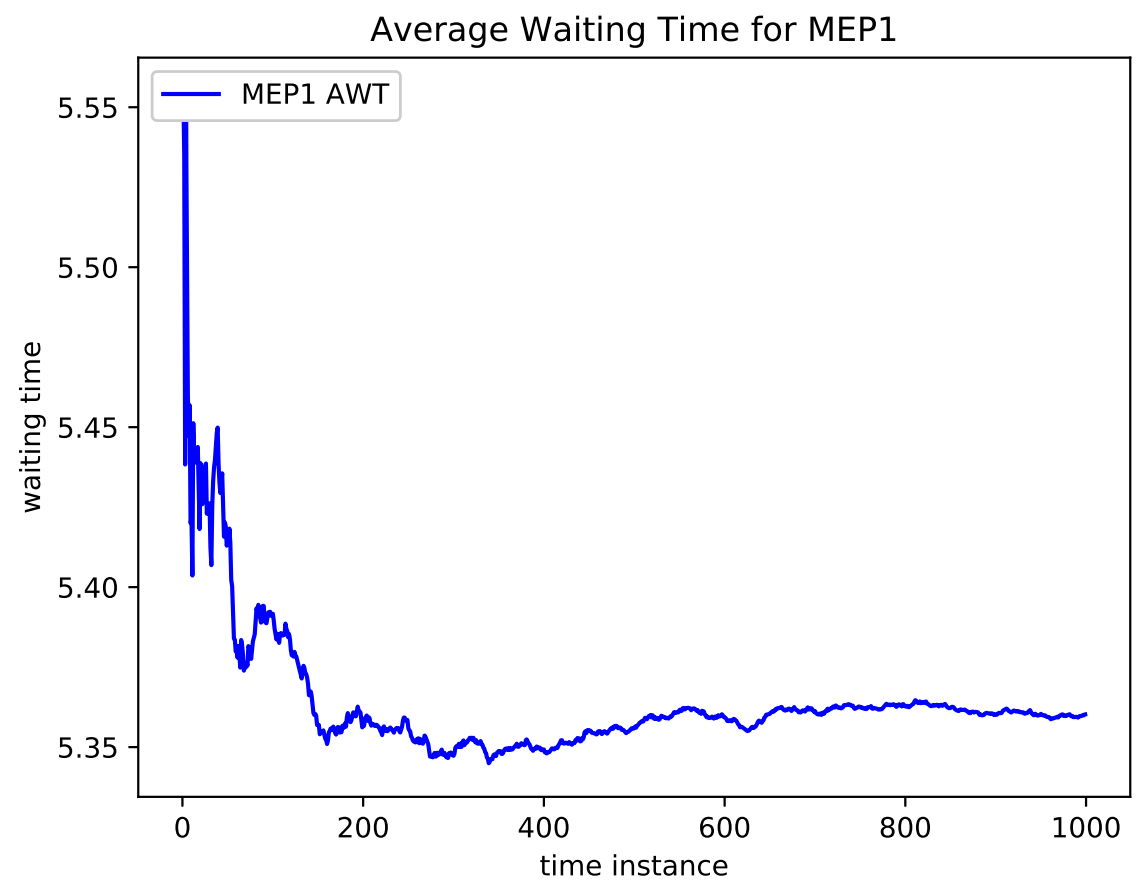

Figure 4.1: The Average Waiting Time for the "Do Nothing" Policy, MEP1, for an ensemble of 200 experiments for the case of the Exp distribution.

elevator dropped-off the passenger at the destination location, but instead of staying at the destination floor as a parking floor, the elevators moved to predetermined parking floors that are associated with the elevators after every service. The corresponding algorithm is given in Algorithm 10.

Algorithm 11 shows how the optimal parking floors were calculated. This was done by exhaustively searching across the combination of floors which minimized a function representing the expected waiting time. The extended equation for this is as follows:

$$
T\left(f_{1}, \ldots, f_{e}\right)=\sum_{y=1}^{m p_{1}}\left|y-f_{1}\right| * g(y)+\ldots+\sum_{y=m p_{e-1}}^{n}\left|y-f_{e}\right| * g(y),
$$

where $f_{1}$ to $f_{e}$ are the floors selected as parking floors for the set of available elevators, $n$ is the number of floors in the building, $g(y)$ is the probability of receiving a call 


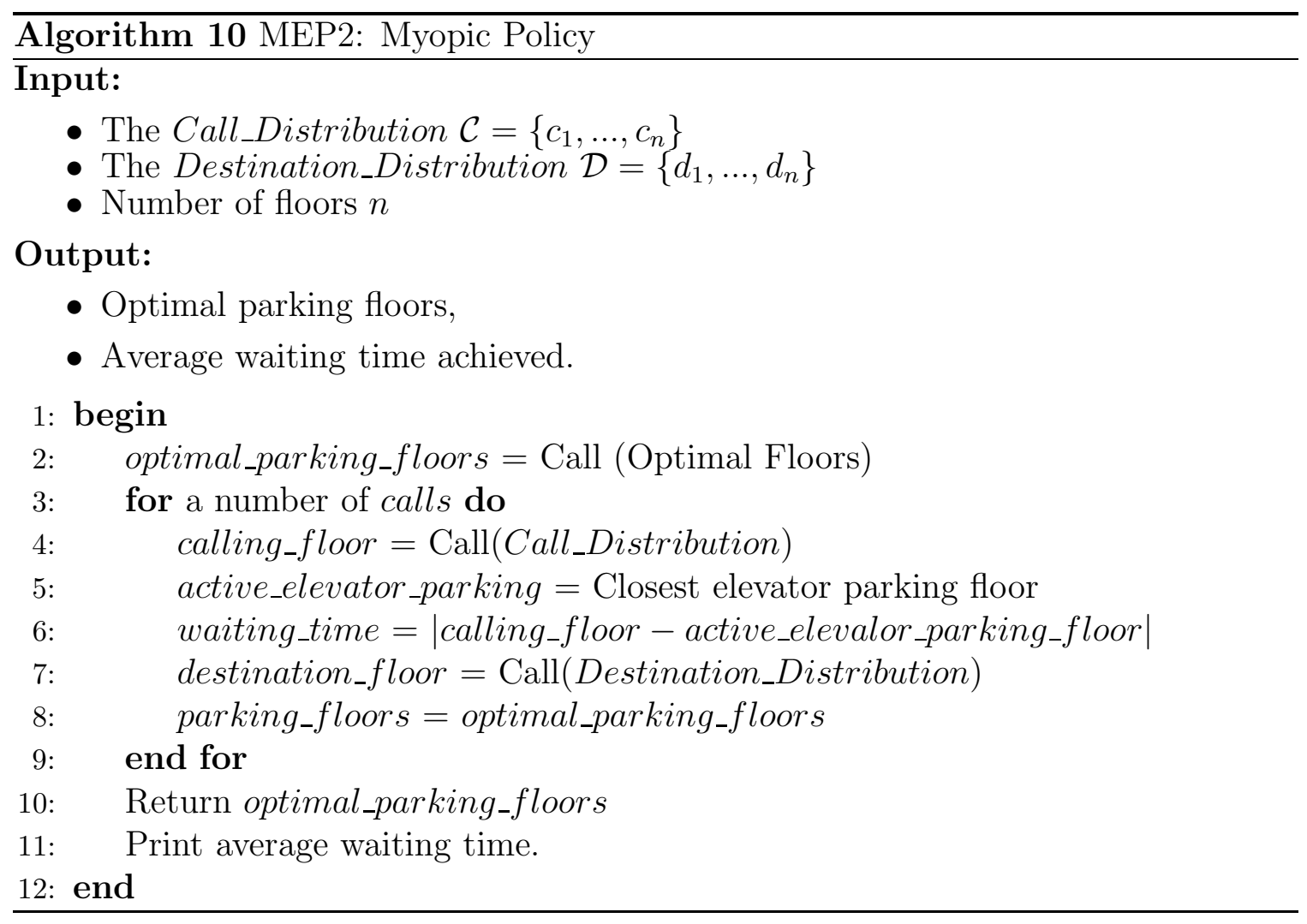


from the floor $y, m p_{e-1}$ is the midpoint between the two consecutive parking floors $f_{e-1}$ and $f_{e}$. In our study, as specified earlier, we employed 2 and 3-elevator models.

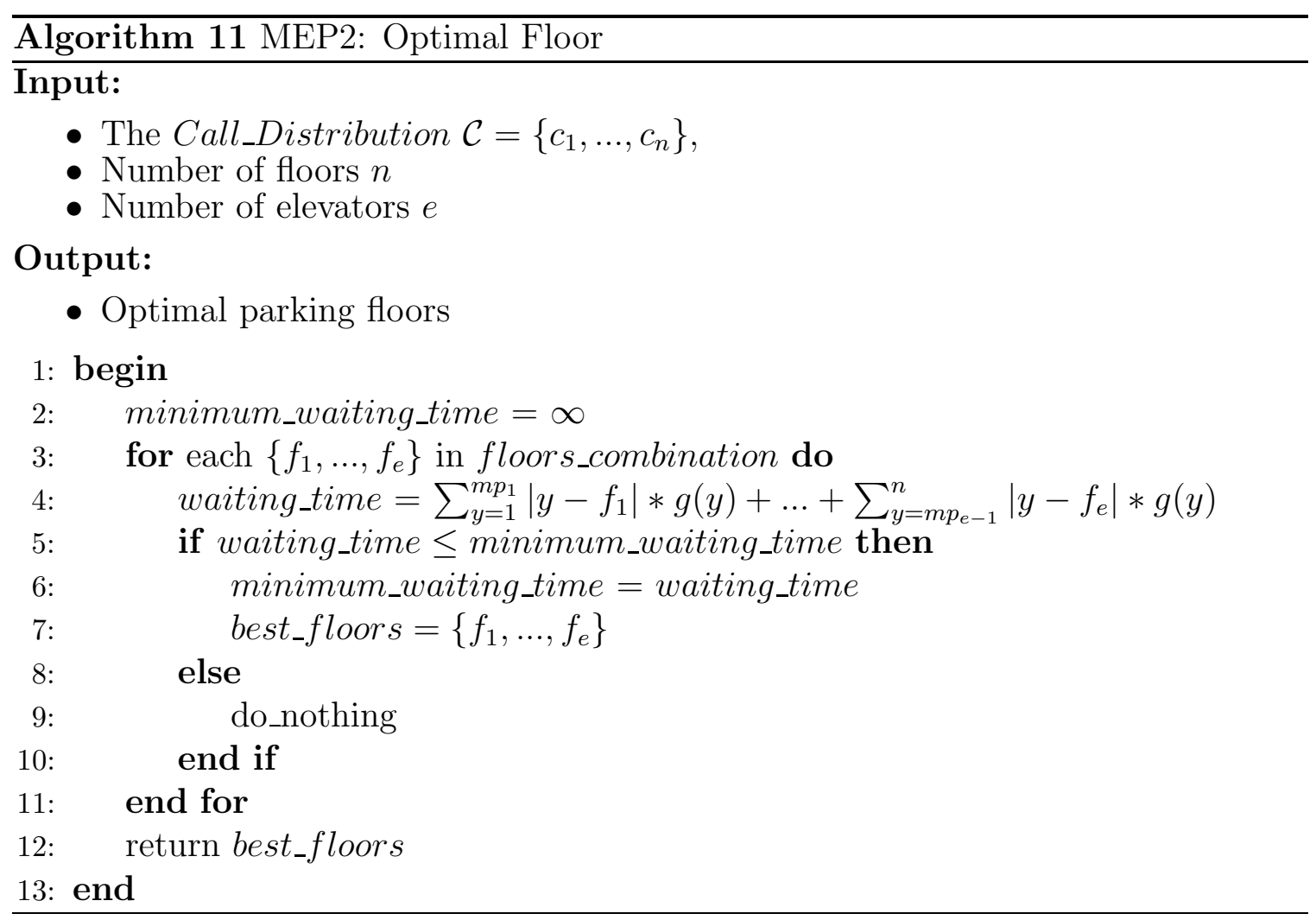

The main disadvantage of this policy is that, as in SEP2, it requires the a priori knowledge of $\mathcal{C}$ to calculate the expected waiting time for each combination of floors.

\section{Simulation Results}

To test the performance of MEP2 or the myopic policy, we used the same building settings mentioned previously. For the sake of comparison, we will include the results for the same settings that we showed the results for, in the case of MEP1. In Table 4.2, we use the same legend as in Table 4.1 with the addition of the row captioned BestFloors, which is the "best set of parking floors" calculated.

From Table 4.2, where we considered the case of 12 floors, we can see that the performance of MEP2 exceeded that of MEP1, especially when the distribution was skewed towards a specific floor, as in Exp and InvExp. The AWT in the Exp scenario 
decreased significantly from 5.36 to 0.36 . Unlike MEP1, the algorithm determined a set of floors that the elevators would always wait at for the next call, which were the first and the third floors in the Exp setting.

Similarly, in the InvExp scenario, the results showed a huge decrease in the $A W T$, and the algorithm determined that the best parking floors were floors 10 and 12 , and that resulted in an $A W T$ of 0.357, down significantly from 5.37. Both Exp and InvExp scenarios yielded marked decreases in the $A W T$ of about $93 \%$.

In the Gaussian and Bimodal cases, the improvements in the performance were not as significant as in the previous distributions, as most of the calls were more distributed across all the floors. So it is reasonable to expect a higher $A W T$ because more calls originated from other floors. For the Gaussian case, the algorithm picked floors 4 and 8 to be the optimal and that reduced the $A W T$ to 1.25 from 2.62. For the Bimodal case, floors 4 and 9 were selected to be optimal parking floors, and that reduced the $A W T$ to 1.07 from 2.67. In both distributions, the decrease in waiting time was around $60 \%$.

In Figure 4.2, we plot the results of the $A W T$ for an ensemble of 200 experiments for the Exp distribution, where the number of passenger calls was 1,000. Observe that for the MEP2, the value converged to the final terminal value of $A W T$ from the starting configuration in less than 50 calls, and this is very reasonable since it calculates the best floors before the simulation processes the calls.

In Figure 4.3, one can observe the difference in the performance between the two policies, MEP1 and MEP2, and how MEP2 outperformed MEP1 to produce a very low $A W T$ in comparison with MEP1.

Just as in the case of SEP2, the problem with MEP2 is that to achieve these results, the algorithm needs to know $\mathcal{C}$, a priori, to calculate the set of best parking floors. In the next section, we will show how one can obtain even more superior results or (close to the optimal results) with LA-based solutions that do not require this knowledge.

Additional experimental results for other building parameters and for other relevant distributions are shown in Appendix B. 
Table 4.2: Simulation results for a 12-floor building for the policy MEP2 for an ensemble of 200 experiments. The results reported are the average waiting times for passengers in terms of number of floors for the elevator car to travel so as to reach the next call from the parked location.

\begin{tabular}{c|cccc}
\hline Dist & Exp & InvExp & Gaussian & Bimodal \\
\hline$c_{1}$ & 0.59344245 & 0.00002978 & 0.02592411 & 0.01270883 \\
$c_{2}$ & 0.24127569 & 0.00007324 & 0.04518336 & 0.03860891 \\
$c_{3}$ & 0.09809538 & 0.00018013 & 0.07046903 & 0.07525205 \\
$c_{4}$ & 0.0398826 & 0.00044306 & 0.09834746 & 0.09457958 \\
$c_{5}$ & 0.01621506 & 0.00108974 & 0.12282111 & 0.08017525 \\
$c_{6}$ & 0.00659255 & 0.00268033 & 0.13725493 & 0.06220755 \\
$c_{7}$ & 0.00268033 & 0.00659255 & 0.13725493 & 0.08440493 \\
$c_{8}$ & 0.00108974 & 0.01621506 & 0.12282111 & 0.14232761 \\
$c_{9}$ & 0.00044306 & 0.0398826 & 0.09834746 & 0.17475873 \\
$c_{10}$ & 0.00018013 & 0.09809538 & 0.07046903 & 0.13967666 \\
$c_{11}$ & 0.00007324 & 0.24127569 & 0.04518336 & 0.07169797 \\
$c_{12}$ & 0.00002978 & 0.59344245 & 0.02592411 & 0.02360195 \\
\hline BestFloors & $\langle 1,3\rangle$ & $\langle 10,12\rangle$ & $\langle 4,8\rangle$ & $\langle 4,9\rangle$ \\
\hline AWT & 0.3597 & 0.3568 & 1.2511 & 1.0651 \\
\hline
\end{tabular}




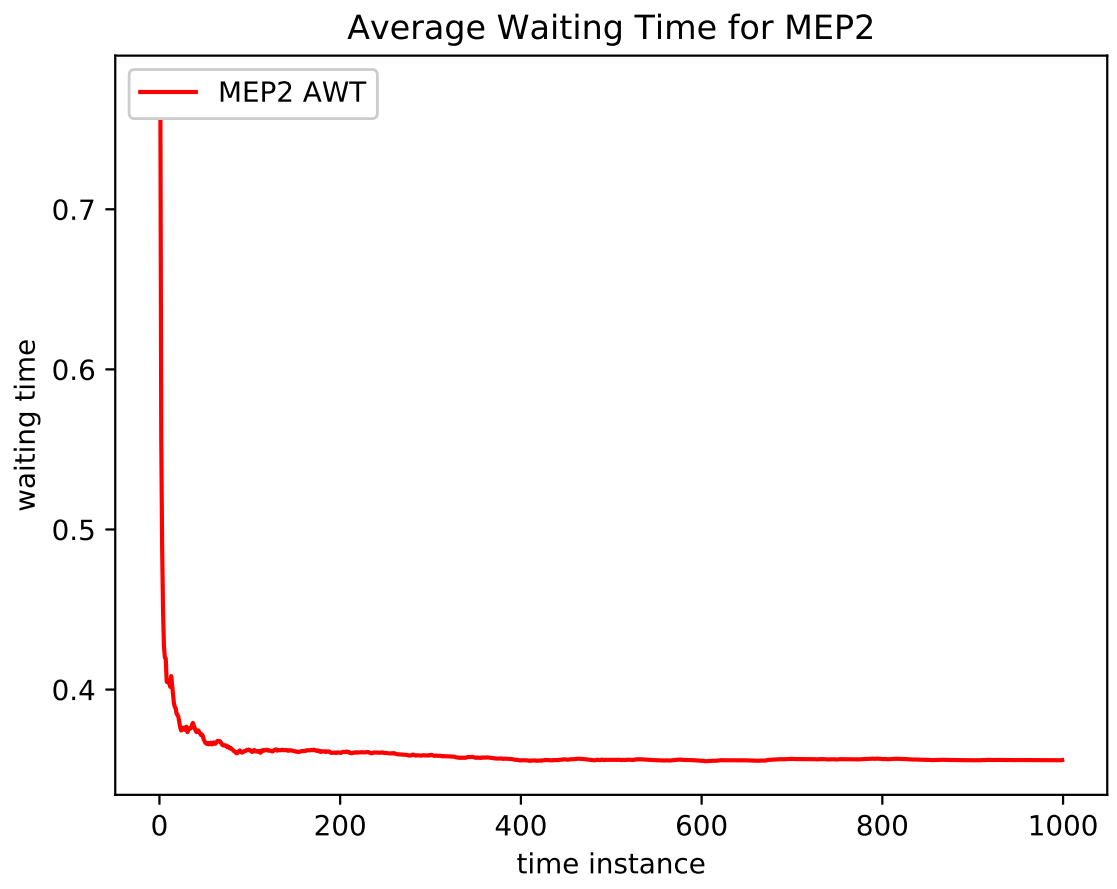

Figure 4.2: The Average Waiting Time for the Myopic Policy, MEP2, for an ensemble of 200 experiments for the case of the Exp distribution.

\subsection{LA-Based Solutions}

In this section, we are going to present our proposed LA-based solutions for the MEP. We are first going to consider how the problem was modelled, and what has changed from the previous single elevator scenario so that the LA-based solution could be adaptable to the multi-elevator setting. The first solution is an $L_{R I}$-based solution, and it is referred to as MEP3. The second one is its enhancement using the pursuit concept to give us the $P L_{R I}$-based solution, referred to as MEP4. In each case, we will present our solution and submit the corresponding results. We then will compare our models' results with the benchmarks mentioned previously. 


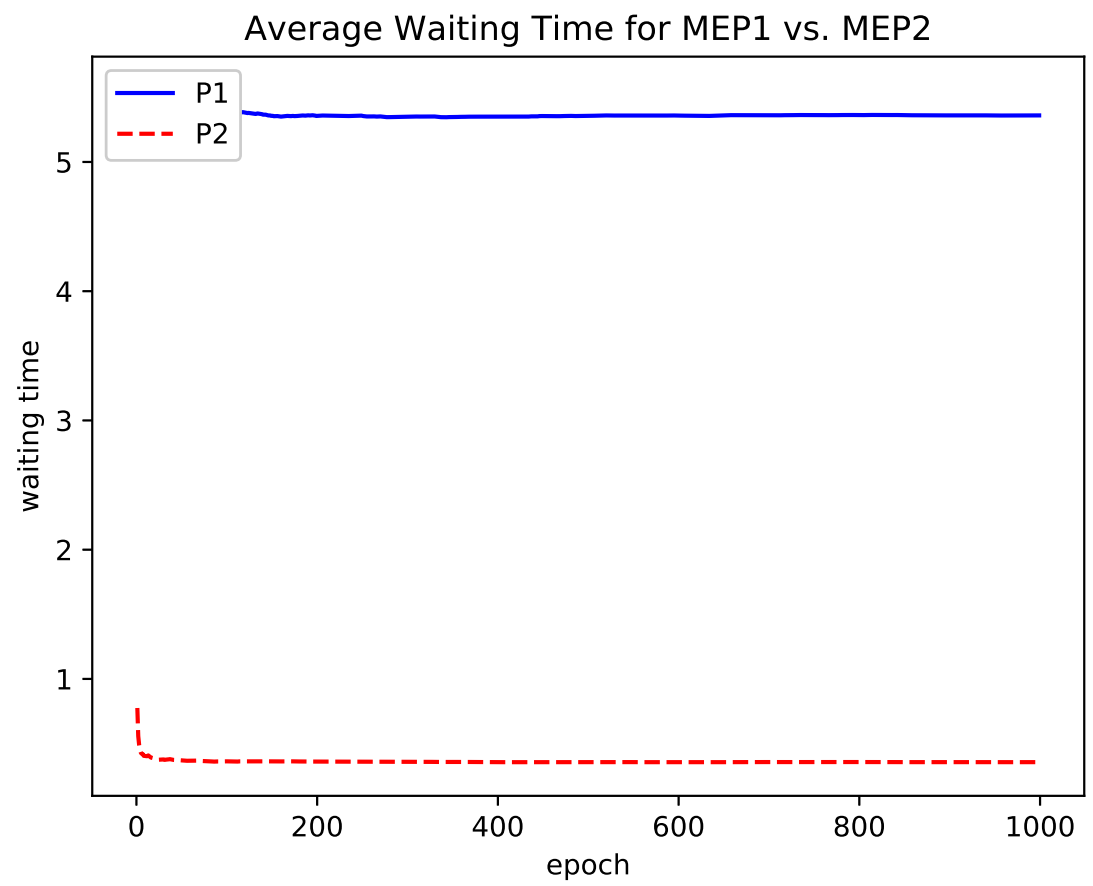

Figure 4.3: The Average Waiting Time for the MEP1 vs. MEP2, for an ensemble of 200 experiments for the case of the Exp distribution.

\subsubsection{Problem Modelling}

As mentioned in Section 3.5.1, any LA-based structure consists of an Environment and the LA itself. Unlike the SEP, in the MEP we have to take into consideration the fact that we are having multiple elevators, and this renders the problem to be more complicated. To model the problem, we have to define what would constitute the LA, and what the Environment would do. The Environment acts in a manner similar to how it performed in the SEP Environment.

On the other hand, the LA design differs inasmuch as we could employ two models. In the first model, the actions are specified as a tuple of a combination of floors, and the issue of selecting the floors as seen as a single decision. However, this method requires a considerable amount of memory as the number of elevators increases, because it involves an exponentially-increasing quantity in order to store each combination of floors. The second model requires having different LA for each elevator. This 
paradigm saves memory as it requires a linearly increasing amount of space. This is the paradigm used in our thesis.

Each LA acts as an elevator's controller, which picks one of the available floors at which it will park at so as to wait for the next call. Moreover, all the LAs (i.e., the controller), will share the same Environment as they all operate in the same building.

\subsection{2 $\mathrm{L}_{R I}$-Based Solution: MEP3}

Our first proposed solution is based on the $L_{R I}$ scheme where the LA updates the probabilities of the actions based on the feedback received from the Environment. It updates them when it receives a reward feedback, and keeps them unchanged when it receives a penalty feedback.

The corresponding algorithm of MEP3 is presented in Algorithm 12. The simulation starts by having the elevator randomly selecting initial parking floors in the building. Each elevator acts independently from the other, and thus, each one has its own action probability for the parking floors, and both are initially created in an equiprobable manner. The Environment generates a call floor and a destination floor using the associated distributions $\mathcal{C}$ and $\mathcal{D}$ respectively. Thereafter, the closest elevator to the calling floor will be dispatched to service that passenger, and its waiting time is used to determine whether the parking floor that the elevator came from was good or not. The corresponding feedback from the Environment is presented in Algorithm 13.

Once the Environment sends the feedback to the elevator, only the active elevator's action probabilities are updated. After the active elevator drops off the passenger, all elevators pick a parking floor, each according to its action or parking probabilities ${ }^{1}$. The corresponding updating algorithm is presented in Algorithm 14.

After this, the procedure repeats itself until the LA converges to a set of floors that correspond to the number of elevators in the building.

\footnotetext{
${ }^{1}$ This is done to prevent any of the elevators from being "stuck" at one position without being called during the whole simulation.
} 


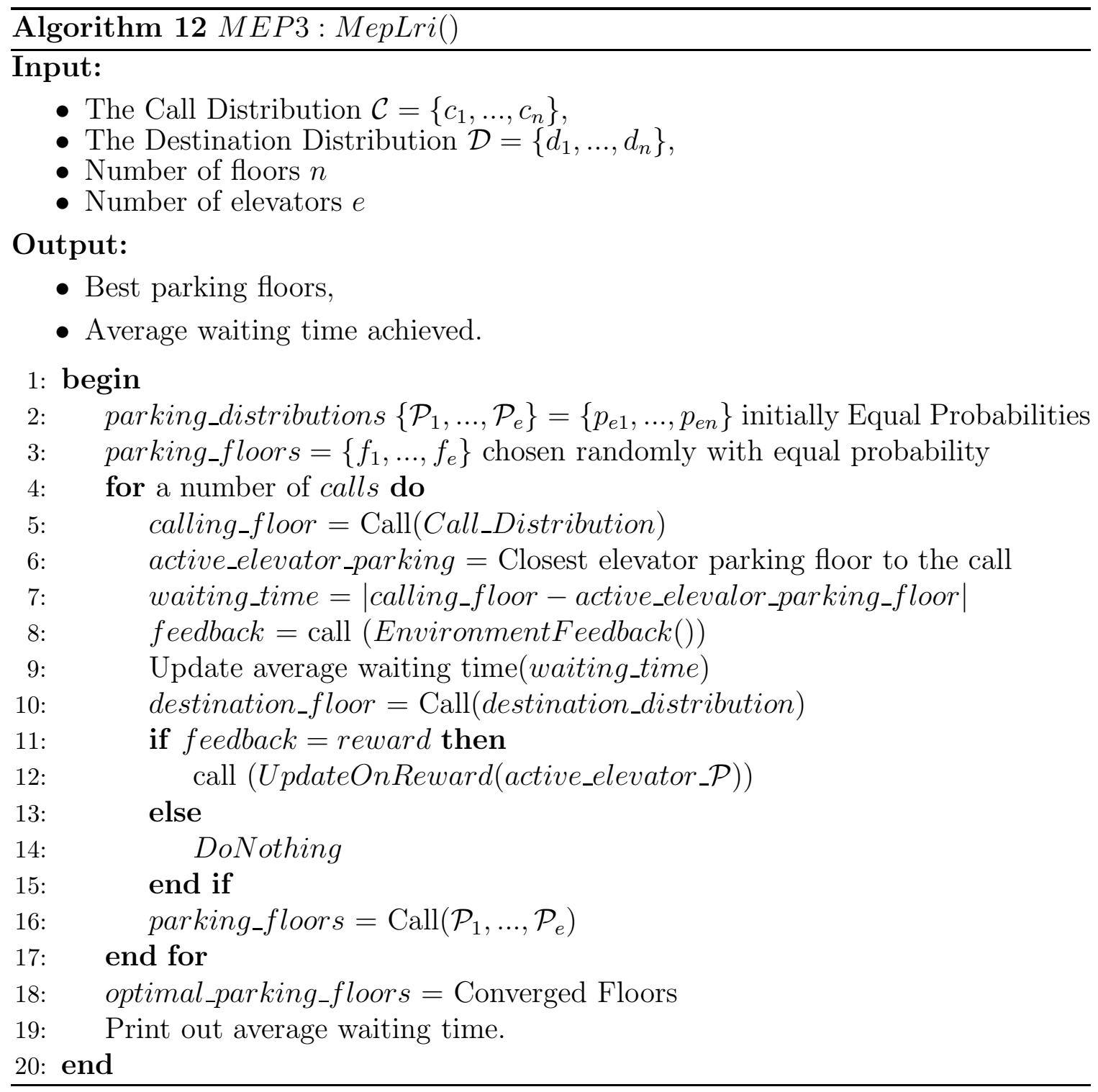



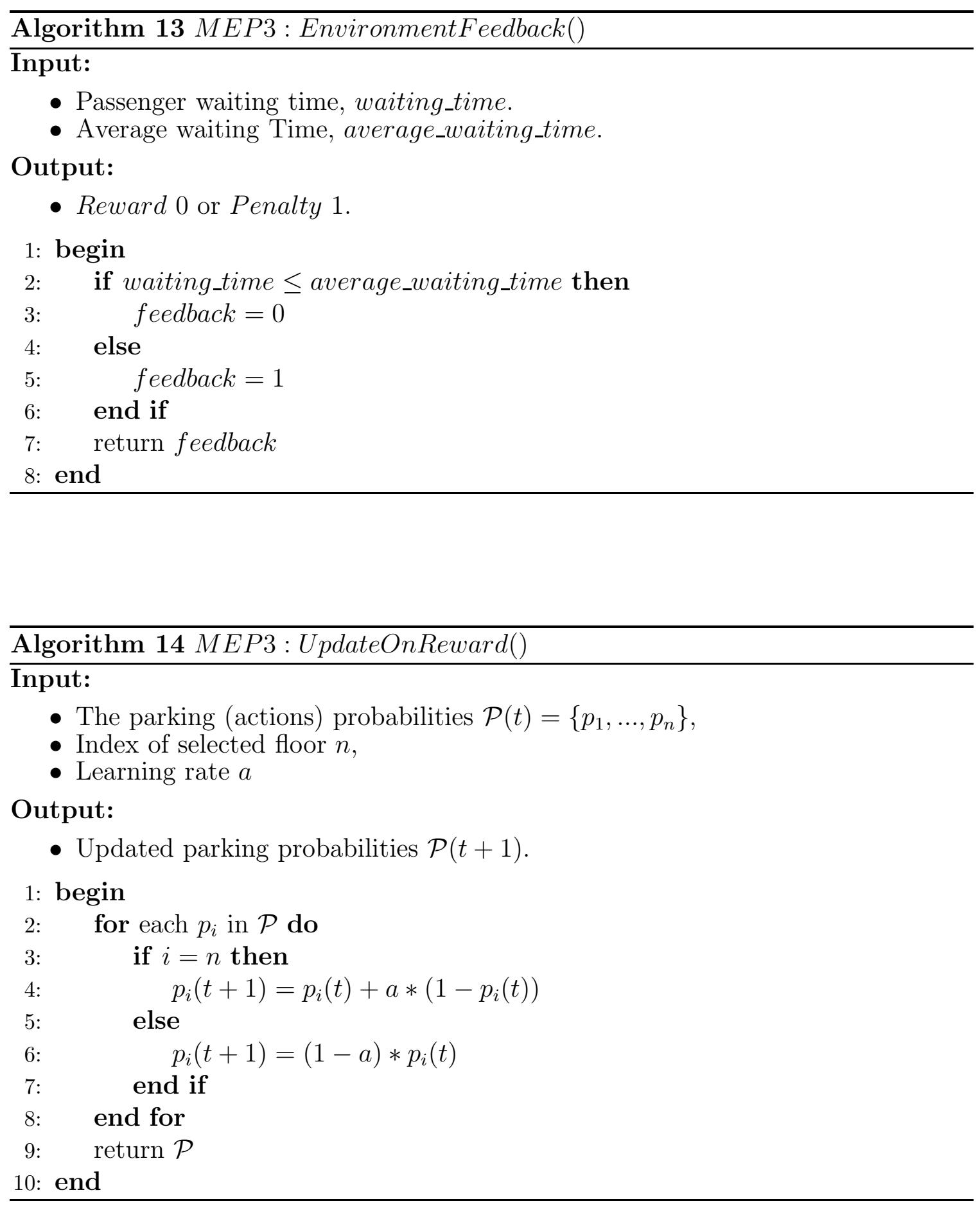


\section{Simulation Results}

To test our proposed solution, MEP3, we used the same building settings that were used earlier in Sections 4.4.1 and 4.4.2 for the purpose of a fair comparison. We discuss the results below in Table 4.3.

From Table 4.3, one can observe that our proposed solution yielded the optimal (or near optimal) floors for the various distributions, with a value that was close to the optimal $A W T$.

Consider the first scenario with the exponential distribution, Exp. The results obtained showed that for MEP3, the LA were able to converge to an optimal floor for one elevator, which is the first floor, and to a floor in the near proximity for the second elevator, which is the third floor. It was thus able to reach a better $A W T$ than MEP1 and very close to MEP2, with an $A W T$ of around 0.78 floors to reach the calling floor.

Similarly, in the second scenario, InvExp, our results showed that MEP3 was able to converge to the best parking locations, which were floors 10 and 12 . Since this distribution is merely the "inverse" of the previous distribution, this was again, very reasonable. This yielded an $A W T$ of 0.76 . Both the first and second scenarios showed a noticeable increase in performance in comparison with the MEP1, and also produced results comparable to the MEP2 without the necessity of knowing $\mathcal{C}$ a priori.

In the third scenario, Gaussian, where most calls were not concentrated and where they were more evenly distributed, the results demonstrated that it achieved a very low $A W T$ of 1.52 when it converged to floors 5 and 8 .

Similarly, for the final scenario, Bimodal, MEP3 was able to produce an even lower value of $A W T$ than MEP1, with an $A W T$ of 1.48 , when it converged to floors 5 and 9. Once again, one can observe here that the impact of MEP3 is very noticeable on the first two scenarios where the calls were skewed towards a specific zone or floor, or when there is a specific pattern.

The overall performance of MEP3 showed a significant decrease in the $A W T$ that was between $42 \%$ to almost $86 \%$ of MEP1's performance. It was also able to produce results that were very close to MEP2, where the optimal solutions were known from the beginning. Instead, MEP3 adapted to the Environment and was able to conclude 
what would be the best possible set of floors to be used as parking floors for the set of available elevators.

Table 4.3: Simulation results for a 12-floor building for the policy MEP3 for an ensemble of 200 experiments. The results reported are the average waiting times for passengers in terms of number of floors for the elevator car to travel so as to reach the next call from the parked location.

\begin{tabular}{c|cccc}
\hline Dist & Exp & InvExp & Gaussian & Bimodal \\
\hline$c_{1}$ & 0.59344245 & 0.00002978 & 0.02592411 & 0.01270883 \\
$c_{2}$ & 0.24127569 & 0.00007324 & 0.04518336 & 0.03860891 \\
$c_{3}$ & 0.09809538 & 0.00018013 & 0.07046903 & 0.07525205 \\
$c_{4}$ & 0.0398826 & 0.00044306 & 0.09834746 & 0.09457958 \\
$c_{5}$ & 0.01621506 & 0.00108974 & 0.12282111 & 0.08017525 \\
$c_{6}$ & 0.00659255 & 0.00268033 & 0.13725493 & 0.06220755 \\
$c_{7}$ & 0.00268033 & 0.00659255 & 0.13725493 & 0.08440493 \\
$c_{8}$ & 0.00108974 & 0.01621506 & 0.12282111 & 0.14232761 \\
$c_{9}$ & 0.00044306 & 0.0398826 & 0.09834746 & 0.17475873 \\
$c_{10}$ & 0.00018013 & 0.09809538 & 0.07046903 & 0.13967666 \\
$c_{11}$ & 0.00007324 & 0.24127569 & 0.04518336 & 0.07169797 \\
$c_{12}$ & 0.00002978 & 0.59344245 & 0.02592411 & 0.02360195 \\
\hline BestFloors & $\langle 1,2\rangle$ & $\langle 11,12\rangle$ & $\langle 6,7\rangle$ & $\langle 5,9\rangle$ \\
\hline AWT & 0.493 & 0.508 & 1.658 & 1.715 \\
\hline
\end{tabular}

Figure 4.4 demonstrates the behaviour of the $A W T$ for MEP3 over the number of calls as it decreased to be a single floor.

As before, additional results for the other settings and distributions are included in Appendix B.

\subsection{3 $\mathrm{PL}_{R I}$-Based Solution: MEP4}

The second LA-based solution, referred to as MEP4, is the $P L_{R I}$-based model. This is based on applying the pursuit concept, where we keep track of the ratios of rewards to the number of times an action was selected, and we use this to enhance the previous MEP3 solution. We observed in Section 3.5.3 that applying the pursuit concept often yielded superior results when compared to the non-pursuit $L_{R I}$-based solution. 


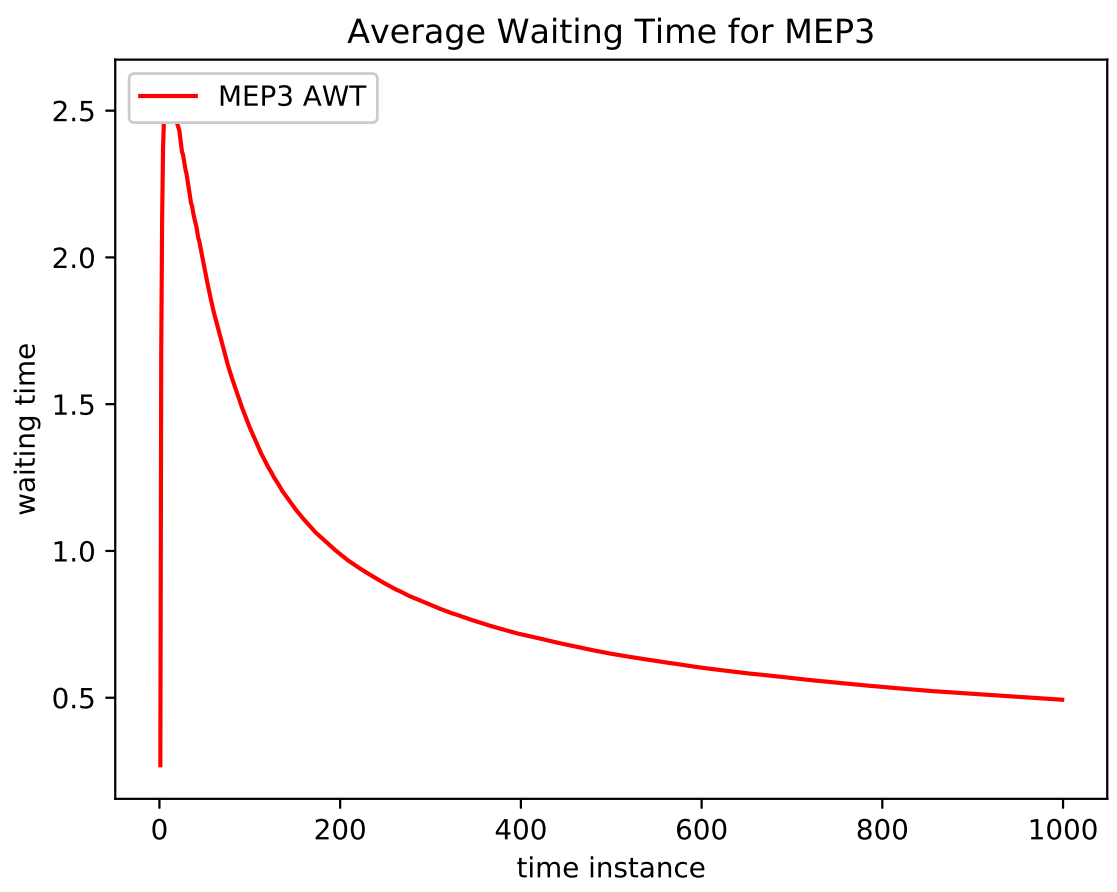

Figure 4.4: The Average Waiting Time for the $L_{R I}$ solution, MEP3, for an ensemble of 200 experiments for the case of the Exp distribution.

The corresponding algorithm for MEP4 is presented in Algorithm 15. As before, the simulation started by selecting each action, which represents the selection of a combination of parking floors for the set of elevators, for a small number of calls, i.e., in our case, 10 times. Thereafter, the Environment evaluated the selected actions and recorded the ratio of the number of times an action was rewarded to the number of times it was chosen. The simulation then proceeded in the same manner as MEP3.

The Environment generated a number of calls and destinations according to the distributions $\mathcal{C}$ and $\mathcal{D}$, and for each call, the closest elevator served the passenger. It then requested the feedback from the Environment, which evaluated the current parking floors using Algorithm 13. Subsequently, the active elevator dropped the passenger off at the destination floor, and then, if the LA of the active elevator received a reward feedback from the Environment, it updated its parking probabilities. On the other hand, it did nothing if it received a penalty feedback. The corresponding 
updating algorithm is presented in Algorithm 16.

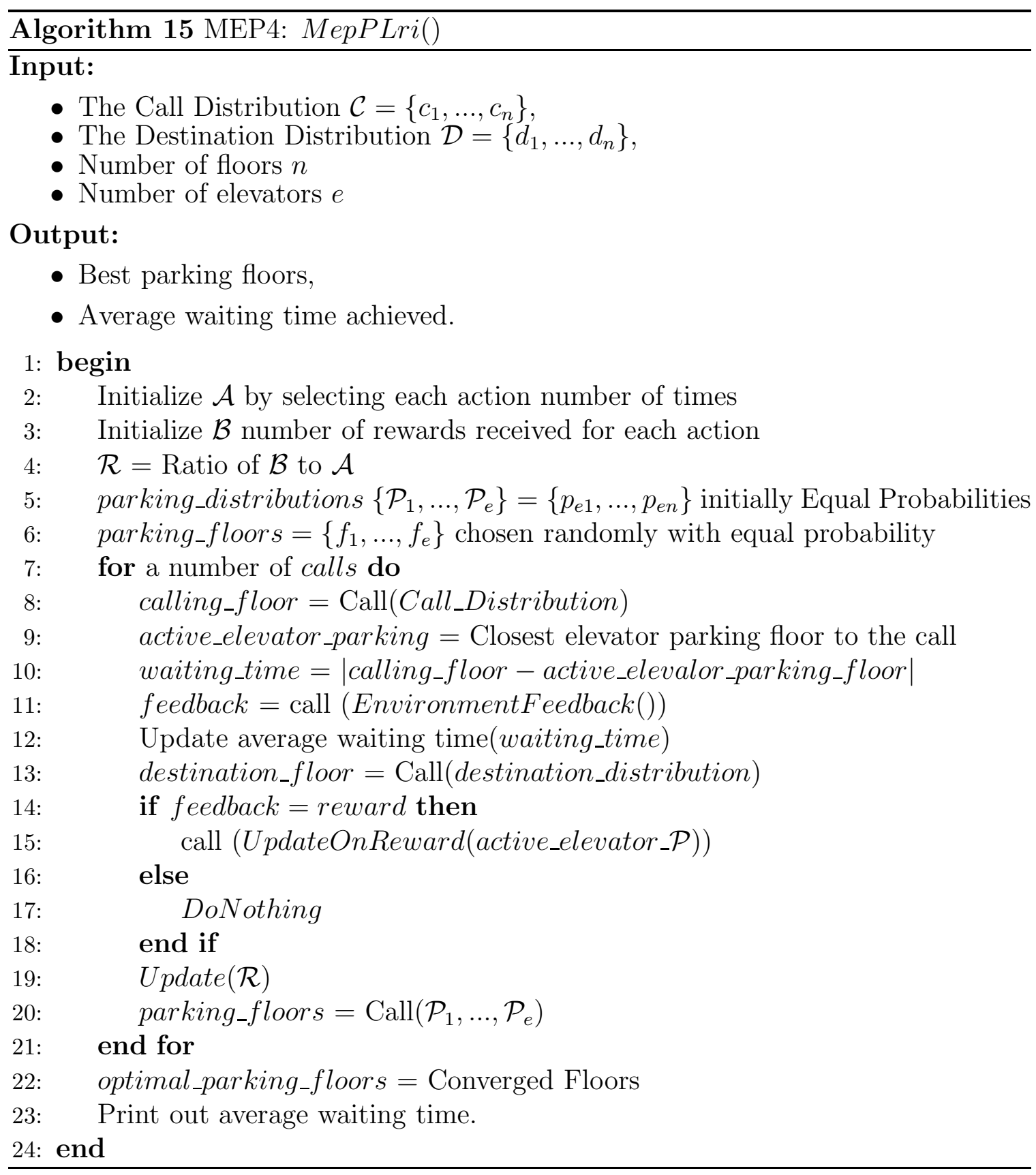

After the active elevator updated the parking probabilities, the LA updated its rewards' estimates for each elevator as per its parking probabilities. 


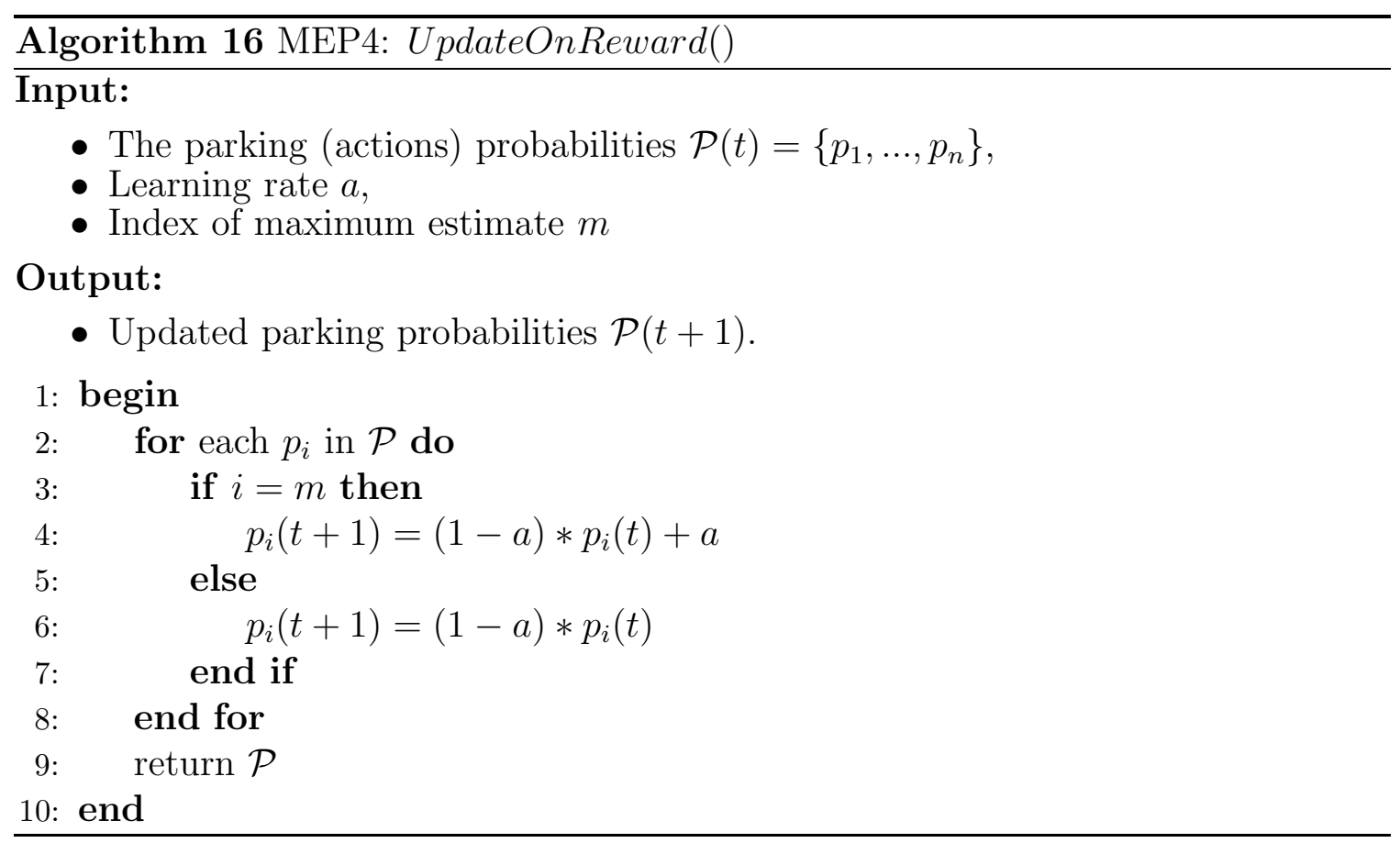

\section{Simulation Results}

To test MEP4, we used the same building settings as in the previous solutions, MEP1, MEP2 and MEP3. Table 4.4 presents the results acquired from the simulations of the four distributions for MEP4.

In the first scenario, Exp, the results obtained showed a slight improvement in the performance with comparison to MEP3. The $A W T$ decreased even more as a result of a faster convergence to the optimal location, and gave even closer results to the optimal values of MEP2 with an $A W T$ of 0.76 down from 0.78 as it converged to the parking floors 1 and 2 .

Similarly, in the second scenario, InvExp, MEP4 showed a superior performance in comparison to MEP1 with a similar decrease with an $A W T$ of 0.87 . Moreover, it converged to the floors 11 and 12, which are very close to the optimal floors.

In the third scenario, Gaussian, the results in MEP4 showed results similar to the performance of MEP3, with an $A W T$ of 1.52, with the parking floors being 5 and 8.

For the final scenario, Bimodal, it was able to produce a slightly lower $A W T$ 
Table 4.4: Simulation results for a 12-floor building for the policy MEP4 for an ensemble of 200 experiments. The results reported are the average waiting times for passengers in terms of number of floors for the elevator car to travel so as to reach the next call from the parked location.

\begin{tabular}{c|cccc}
\hline Dist & Exp & InvExp & Gaussian & Bimodal \\
\hline$c_{1}$ & 0.59344245 & 0.00002978 & 0.02592411 & 0.01270883 \\
$c_{2}$ & 0.24127569 & 0.00007324 & 0.04518336 & 0.03860891 \\
$c_{3}$ & 0.09809538 & 0.00018013 & 0.07046903 & 0.07525205 \\
$c_{4}$ & 0.0398826 & 0.00044306 & 0.09834746 & 0.09457958 \\
$c_{5}$ & 0.01621506 & 0.00108974 & 0.12282111 & 0.08017525 \\
$c_{6}$ & 0.00659255 & 0.00268033 & 0.13725493 & 0.06220755 \\
$c_{7}$ & 0.00268033 & 0.00659255 & 0.13725493 & 0.08440493 \\
$c_{8}$ & 0.00108974 & 0.01621506 & 0.12282111 & 0.14232761 \\
$c_{9}$ & 0.00044306 & 0.0398826 & 0.09834746 & 0.17475873 \\
$c_{10}$ & 0.00018013 & 0.09809538 & 0.07046903 & 0.13967666 \\
$c_{11}$ & 0.00007324 & 0.24127569 & 0.04518336 & 0.07169797 \\
$c_{12}$ & 0.00002978 & 0.59344245 & 0.02592411 & 0.02360195 \\
\hline BestFloors & $\langle 1,2\rangle$ & $\langle 11,12\rangle$ & $\langle 6,8\rangle$ & $\langle 4,9\rangle$ \\
\hline AWT & 0.4334 & 0.446 & 1.464 & 1.448 \\
\hline
\end{tabular}


than MEP3 with an $A W T$ of 1.45 down from 1.47, and converged to floors 4 and 9 . One can observe how the incorporation of the pursuit concept in MEP4 helped to increase the performance and produce better results in most scenarios, and how this improvement is affected by $\mathcal{C}$.

Figure 4.5 displays the behaviour of how the $A W T$ decreased over time for the first scenario, Exp, for MEP4.

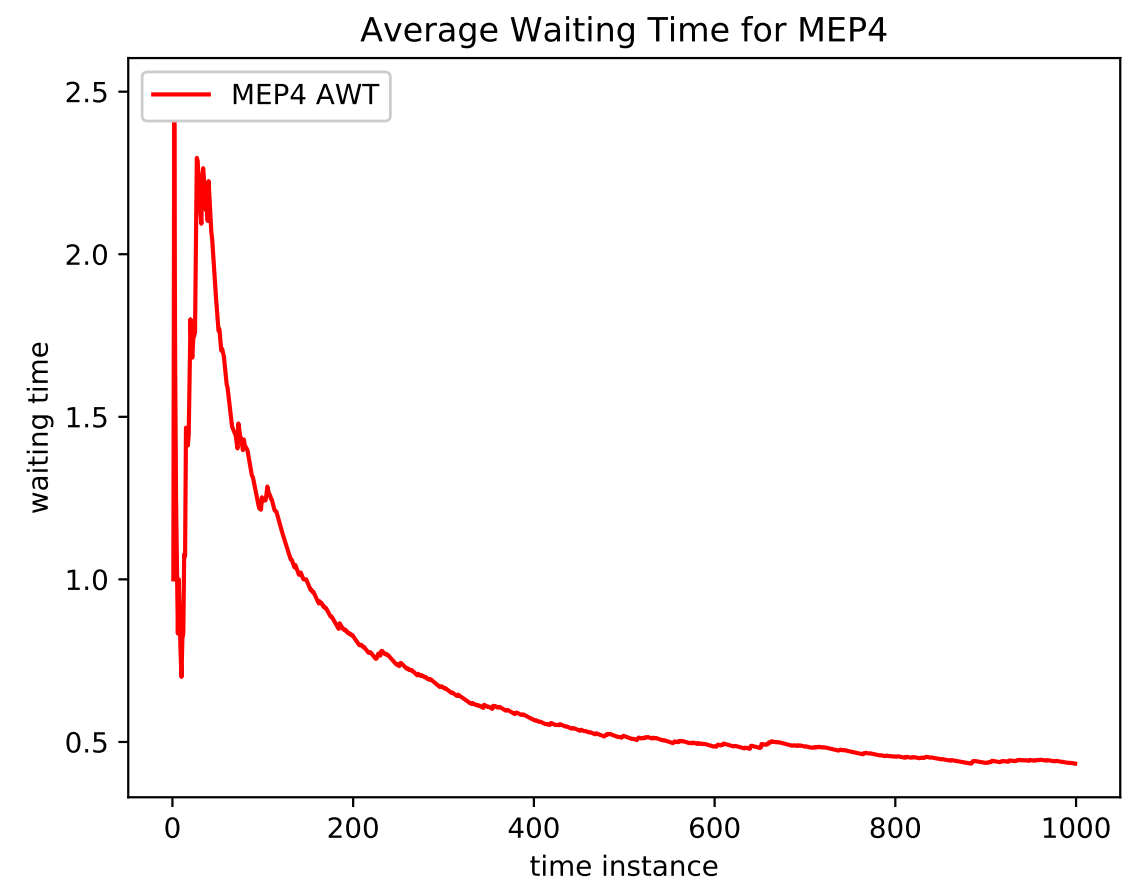

Figure 4.5: The Average Waiting Time for the $P L_{R I}$ solution, MEP4, for an ensemble of 200 experiments for the case of the Exp distribution.

In Figure 4.6, we can see the difference in convergence speed between MEP3 and MEP4, where MEP4 yielded a better performance and faster convergence, which can both be attributed to the pursuit concept.

Again, additional results for the other settings and distributions are included in Appendix B. 


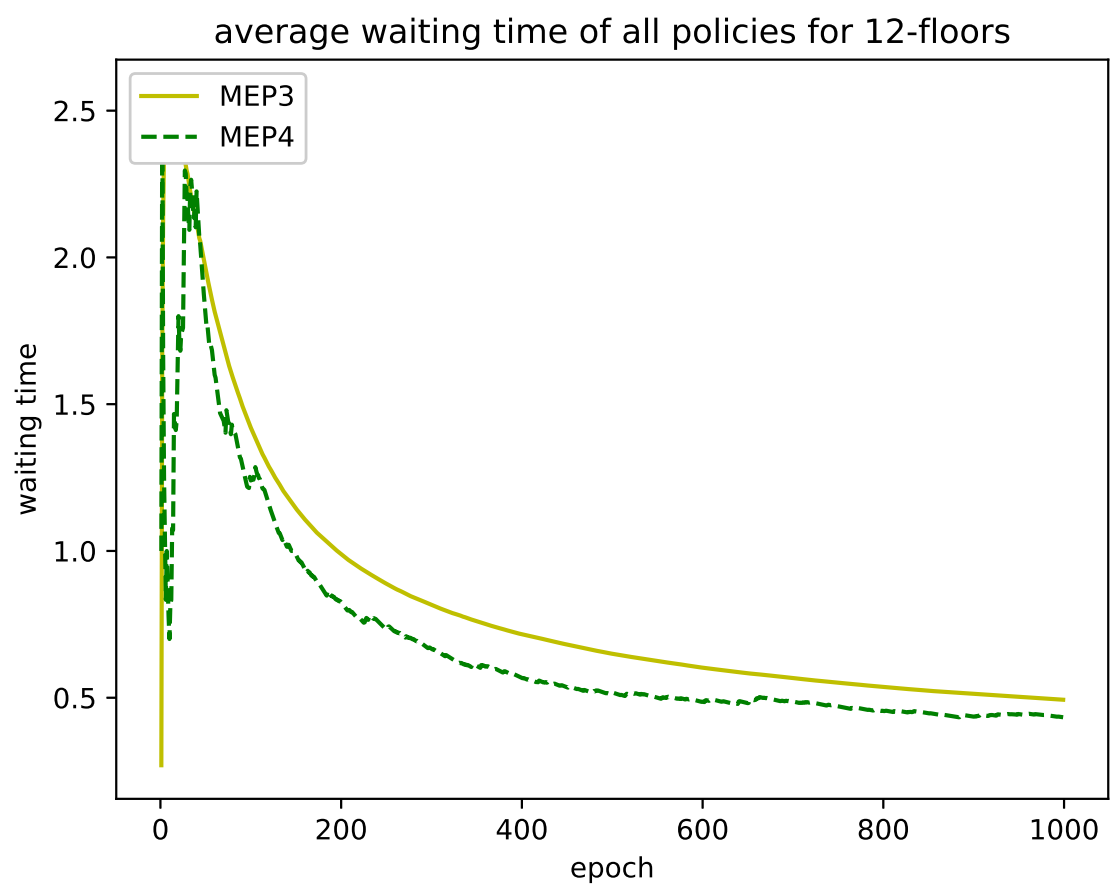

Figure 4.6: The Average Waiting Time for the MEP3 vs. MEP4, for an ensemble of 200 experiments for the case of the Exp distribution.

\subsection{Discussion}

We now comparatively discuss the results of the simulations obtained from the above solutions. In Table 4.5, we submit the results of the four solutions that were discussed, namely, MEP1, MEP2, MEP3 and MEP4. From the table, one can see that MEP1, which we believe is the most popular policy currently used in buildings, performed very poorly in comparison to our proposed solutions, MEP3 and MEP4. The improvement in the average waiting time was more than $40 \%$ and up to $86 \%$. This policy, MEP1, serves as a lower bound for our benchmark, as no solution should perform worse than this.

MEP2 was able to provide the set of optimal parking floors from the beginning, but it required the a priori knowledge of $\mathcal{C}$ for each floor. On the other hand, our LA-based solutions were able to achieve a close-to-optimal $A W T$ and parked at floors 
Table 4.5: Simulation results for the previous and newly proposed solutions for the MEP for an ensemble of 200 experiments for the 12-floor settings. The results are given here as a tuple $(\alpha, \beta)$ where the first field, $\alpha=\left\langle\alpha_{1}, \alpha_{2}\right\rangle$, is the best optimal parking floors for each elevator and the second field, $\beta$ is the $A W T$ in terms of number of floors travelled for the elevator to reach the passenger from the parked locations.

\begin{tabular}{c|cccc|}
\hline Dist & MEP1 & MEP2 & MEP3 & MEP4 \\
\hline Exp & $(-, 5.3604)$ & $(\langle 1,3\rangle, 0.3597)$ & $(\langle 1,2\rangle, 0.493)$ & $(\langle 1,2\rangle, 0.4334)$ \\
InvExp & $(-, 5.3697)$ & $(\langle 10,12\rangle, 0.3568)$ & $(\langle 11,12\rangle, 0.508)$ & $(\langle 11,12\rangle, 0.446)$ \\
Gaussian & $(-, 2.6252)$ & $(\langle 4,8\rangle, 1.2511)$ & $(\langle 6,7\rangle, 1.658)$ & $(\langle 6,8\rangle, 1.464)$ \\
Bimodal & $(-, 2.6770)$ & $(\langle 4,9\rangle, 1.0651)$ & $(\langle 5,9\rangle, 1.715)$ & $(\langle 4,9\rangle, 1.448)$ \\
\hline
\end{tabular}

close to the optimal floors, without the knowledge of $\mathcal{C}$.

MEP3 showed that it was better than MEP1 and recorded an $A W T$ improvement of $85 \%, 86 \%, 42 \%$ and $45 \%$ for the Exp, InveExp, Gaussian and BiModal distributions respectively. Moreover, the results were close to the values achieved by MEP2. Further, the increase in the performance was more than for the SEP models, which demonstrates the effect of having multiple intelligent elevators especially when the number of elevators increases.

As in the case of SEP4, we attempted to improve MEP3 by proposing MEP4 that applied the Pursuit concept. MEP4 was able to achieve slightly better results than MEP3. The algorithm helped the system to converge faster to the optimal locations in most of the cases. It also resulted in a superior $A W T$.

One result that we found from the simulations in the SEP and the MEP is that having a single intelligent elevator could result in a better performance than having multiple elevators where non of them used intelligent policies. The scenario for the 12-floor building system with two elevators is one such example. While in MEP1, the AWT was between 5 and 6 floors, the SEP3 resulted in an average of about only 2 floors. Also, just as in the case of the SEP, the more equally-distributed the calls were, the higher was the value of the $A W T$. On the other hand, the importance of having a good policy becomes evident when the calling distribution is skewed toward a specific region or a specific floor. 
In Figure 4.7, we present the performance of each algorithm and how our proposed LA-based algorithms, MEP3 and MEP4, were able to achieve an AWT that is very close to the optimal solution, MEP2, and how it significantly outperforms MEP1.

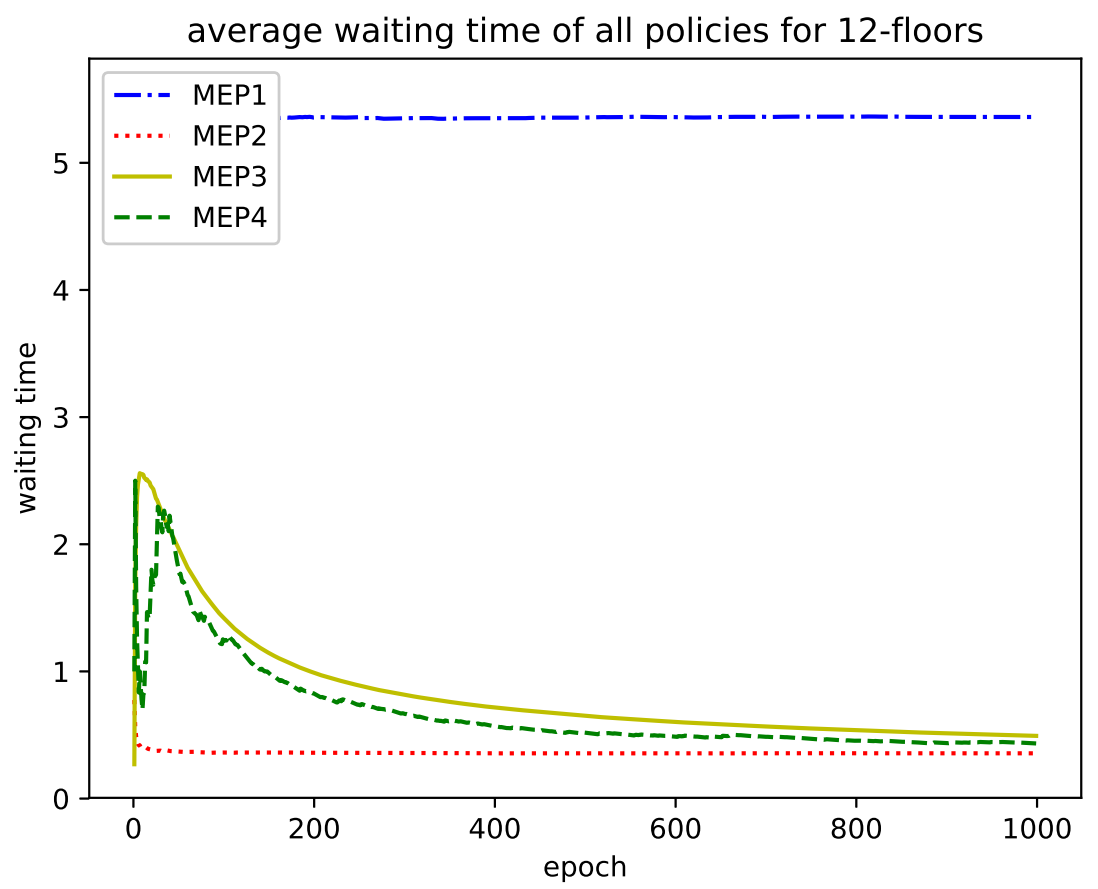

Figure 4.7: The Average Waiting Time for MEP1, MEP2, MEP3 and MEP4 for an ensemble of 200 experiments for the case of the Exp distribution.

\subsection{Conclusion}

In this chapter, we reviewed different parking policies that were used in different building settings and previously-reported solutions for the multi-elevator scenario. We discussed two different solutions and tabulated the experimental results for the various simulations. We then introduced our $L_{R I}$-based solution, MEP3, and showed that it could achieve the optimal parking floor without the need to know $\mathcal{C}$ a priori.

We also presented an improvement on MEP3 to involve the Pursuit concept. This yielded our second solution, MEP4, a $P L_{R I}$-based solution. From the simulation results for MEP4, we demonstrated that it performed even better than MEP3. 
This chapter included our findings and compared the results of our proposed solutions to the previous benchmark solutions. We showed that our LA-based solutions performed better than MEP1 and converged to the optimal floor. They also reduced the $A W T$ to be close to the optimal value with the advantage that they did not require us to know the distributions to find the best set of possible parking floors.

This chapter concludes the research component of our thesis. 


\section{Chapter 5}

\section{Summary, Conclusions and Future Work}

In this chapter, we submit a brief summary of the thesis, record what has been done, and present our conclusions on the results that we have obtained. Finally, we will provide some ideas for potential future relevant work.

\subsection{Summary and Conclusion}

The content of this thesis can be briefly summarized as follows. Our goal was to consider a field of AI that has not been applied to many problems, namely the field of Learning Automata (LA). One of these problems is the elevator problem, where the objective is to determine a set of parking floors that correspond to the elevators in the building, where the elevators would park so as to minimize the waiting time for the passengers. In this context, we mention that there are other real-life problems that possess similar characteristics, which also require some sort of "parking" phenomenon so as to anticipate an event from the environment. We have referred to these as Elevator-Like Problems (ELPs). Our goal was to tackle the real Elevator-named problem so as to provide LA-based solutions that could be generalized and applied to ELPs.

In Chapter 2, we surveyed the concept of "Learning" as considered in Computer 
Science, by addressing its formulation, and by considering how it has been applied to different application domains. We then showed how the field of LA, the main area of focus in this thesis, is related to the concept of Learning. We classified the various LA described in the literature into two different classes according to their characteristics. The first one was the family of Fixed Structure Stochastic Automata (FSSA), and the second one was the family of Variable Structure Stochastic Automata (VSSA), and briefly described each family's performance. After that, we introduced the phenomenon of utilizing the Maximum Likelihood Estimates of the reward probabilities, and explained how the LA can be enhanced using the pursuit phenomenon, i.e., by pursuing the estimated "best" action.

In this chapter, we also surveyed the Elevator Problem and Elevator-Like Problems. Through our survey, we concluded that out of the reviewed papers, only a few considered the problem of determining the optimal parking locations for the elevator problem. We thus formulated our goal so that we could solve that problem, with the intention of optimizing the Average Waiting Time (AWT) for the passengers.

In Chapter 3, we focused on the single elevator scenario, and reviewed different parking policies that we believe could be considered as benchmarks for our proposed solutions. These were the solutions referred to as SEP1 and SEP2, where in SEP1 the elevator uses the current destination floor as the parking floor, and in SEP2 the optimal floors are calculated using calling distribution $\mathcal{C}$, assumed to be known $a$ priori. We then introduced our two LA-based solutions, i.e., SEP3, which is an $L_{R I^{-}}$ based solution, and SEP4, which is an enhancement on SEP3 and which involves the pursuit concept, the $P L_{R I}$-based solution. We demonstrated how SEP3 and SEP4 performed, and reported a comparison with the benchmark models. We also showed that their results were close to the optimal results with the advantage of not knowing the call distributions a priori.

In Chapter 4, we expanded our focus to cover the multi-elevator scenario where we generalized SEP1 and SEP2 to create MEP1 and MEP2 respectively. These served as benchmarks for our proposed algorithms in the multi-elevator case. Thereafter, we proposed our LA-based models for the MEP and we provided two solutions which were MEP3, an $L_{R I}$-based solution, and MEP4, a $P L_{R I}$-based solution. The results 
showed that they performed significantly better than MEP1 and produced results close to the optimal results provided by MEP2.

\subsection{Conclusion and Future Work}

As concluding results, we state the following:

- We surveyed the specific subfield of AI, namely the field of Learning Automata (LA), and reported that it has not been used to solve the elevator problem.

- We identified two different models of computations for the problem, where the first requires the knowledge of the calling distributions and the second does not need this information.

- We modelled the problem in a way such that it could be solved using LA-based approaches.

- We introduced the Linear Reward-Inaction $\left(L_{R I}\right)$-based solutions for the cases of single elevators and multi-elevators, and referred to them as SEP3 and MEP3 respectively.

- We also presented an improvement on the above solutions by including the socalled Pursuit phenomenon. This led to the $P L_{R I}$-based solutions referred to as SEP4 and MEP4 for the single-elevator and multi-elevator settings respectively.

- We showed that LA-based solutions can adoptively solve elevator-like problems without requiring the prior knowledge of any distributions.

- The proposed LA-based solutions provided results that were near-optimal.

The research of studying the elevator problem and ELPs, and by solving them using LA, opened new questions regarding the applications of LA, and queries related to the elevator problem itself. Two of the areas that were not covered in this research, and which can be explored in future research are the following: 
- The question of using LA to determine the best parking policy when the scheduler incorporates different dispatching schemes, can lead to more complicated and constrained settings. These could attempt to work with different optimization criteria based on costs, energy savings and time. This question is yet unanswered.

- The task of studying the elevator problem when it operates in a non-stationary environment is also very complicated and remain open. We believe that by combining multiple patterns, one could obtain LA solutions for these more complicated scenarios and for time-varying settings.

- Another relatively simple exercise will be to obtain the results for other types of distributions, for example, those that resemble early-morning and late-evening traffic patterns.

- The question of having elevators use LA-based solutions to move multiple passengers, and with various car capacities, is also open. 
Appendices 


\section{Appendix A}

\section{SEP Results}

This appendix will include all the results and plots acquired from the simulations done for all the building settings, and for all the pertinent distributions for the case of the SEP. The notations, terminologies and legends are as described in Chapter 3.

Table A.1: Simulation results for the previous and newly proposed solutions for the SEP for an ensemble of 200 experiments for the 8-floor settings. The results are given here as a tuple $(\alpha, \beta)$ where the first field, $\alpha$, is the best optimal parking floor and the second field, $\beta$ is the $A W T$ in terms of number of floors travelled for the elevator to reach the passenger from the parked location.

\begin{tabular}{c|cccc|}
\hline Dist & SEP1 & SEP2 & SEP3 & SEP4 \\
\hline Exp & $(-, 3.4080)$ & $(1,0.673)$ & $(2,0.919)$ & $(1,0.783)$ \\
InvExp & $(-, 3.4086)$ & $(8,0.760)$ & $(7,0.930)$ & $(8,0.782)$ \\
Gaussian & $(-, 2.508)$ & $(5,1.747)$ & $(5,1.919)$ & $(4,1.889)$ \\
Bimodal & $(-, 2.423)$ & $(5,1.473)$ & $(6,1.586)$ & $(6,1.557)$ \\
\hline
\end{tabular}




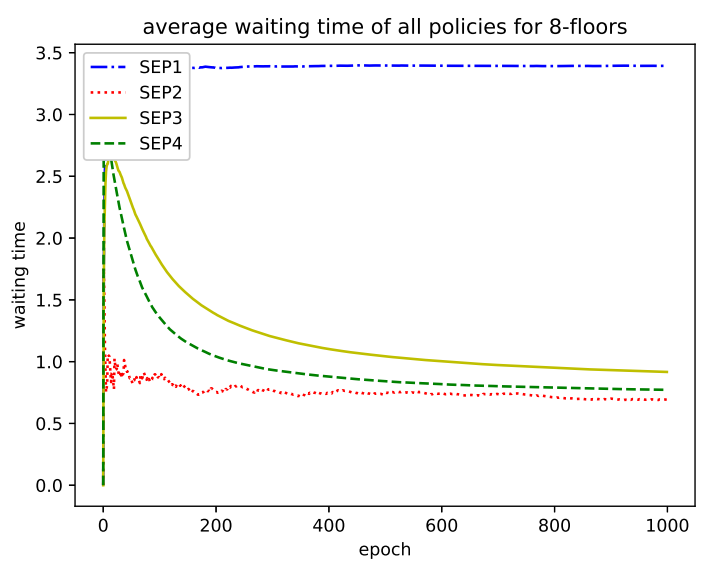

Figure A.1: The Average Waiting Time for SEP1, SEP2, SEP3 and SEP4 for an ensemble of 200 experiments for the case of the Exp distribution for an 8-floor setting.

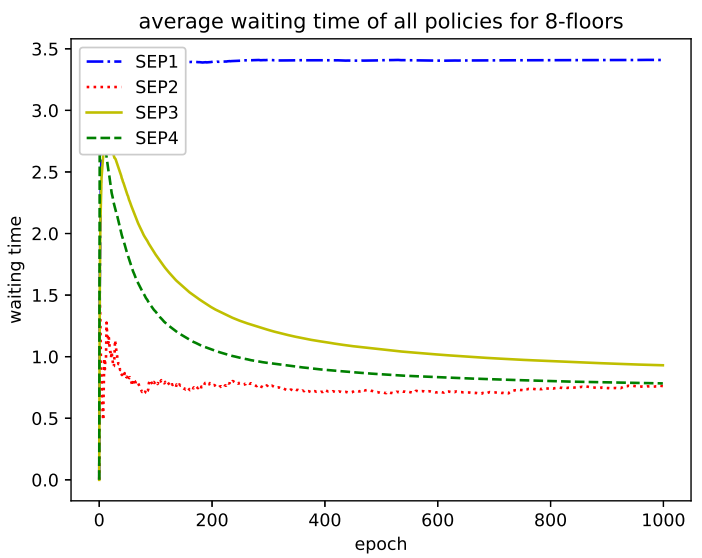

Figure A.2: The Average Waiting Time for SEP1, SEP2, SEP3 and SEP4 for an ensemble of 200 experiments for the case of the InvExp distribution for an 8-floor setting. 


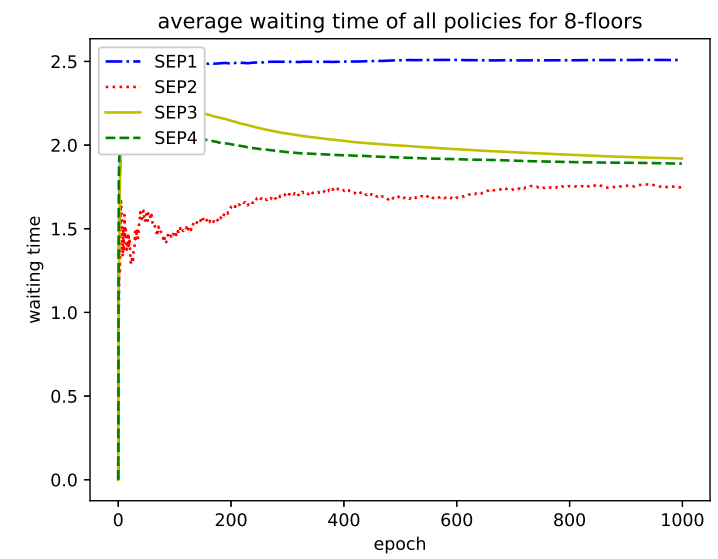

Figure A.3: The Average Waiting Time for SEP1, SEP2, SEP3 and SEP4 for an ensemble of 200 experiments for the case of the Gaussian distribution for an 8-floor setting.

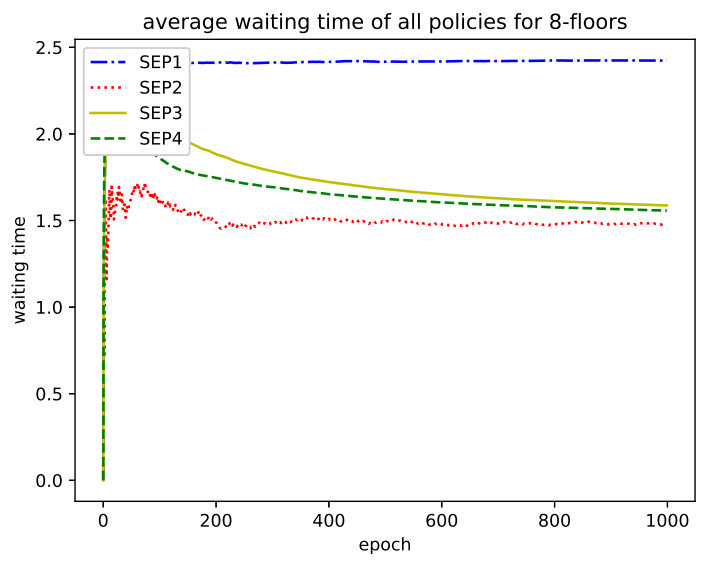

Figure A.4: The Average Waiting Time for SEP1, SEP2, SEP3 and SEP4 for an ensemble of 200 experiments for the case of the Bimodal distribution for an 8-floor setting. 
Table A.2: Simulation results for the previous and newly proposed solutions for the SEP for an ensemble of 200 experiments for the 16 -floor settings. The results are given here as a tuple $(\alpha, \beta)$ where the first field, $\alpha$, is the best optimal parking floor and the second field, $\beta$ is the $A W T$ in terms of number of floors travelled for the elevator to reach the passenger from the parked location.

\begin{tabular}{|c|c|c|c|c|}
\hline Dist & SEP1 & SEP2 & SEP3 & SEP4 \\
\hline $\operatorname{Exp}$ & $(-, 7.352)$ & $(1,0.705)$ & $(2,1.502)$ & $(1,0.897)$ \\
\hline InvExp & $(-, 7.342)$ & $(16,0.639)$ & $(15,1.557)$ & $(16,0.922)$ \\
\hline Gaussian & $(-, 4.583)$ & $(9,2.375)$ & $(8,2.669)$ & $(8,2.617)$ \\
\hline Bimodal & $(-, 4.894)$ & $(11,2.879)$ & $(10,3.213)$ & $(11,3.113)$ \\
\hline
\end{tabular}

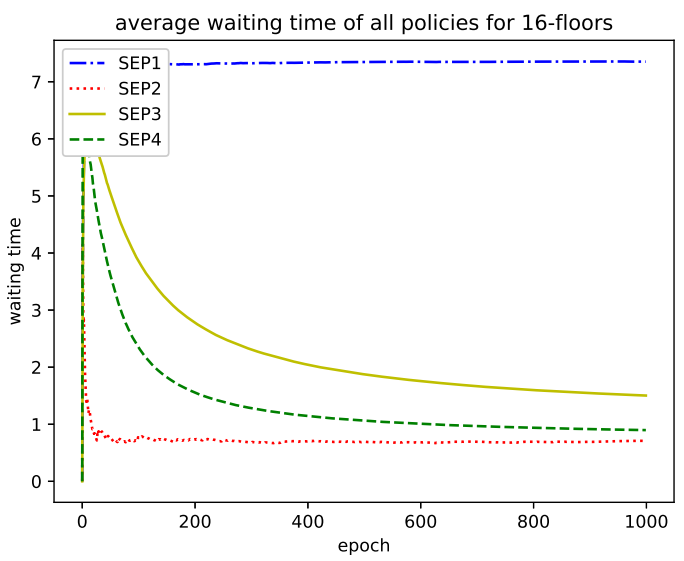

Figure A.5: The Average Waiting Time for SEP1, SEP2, SEP3 and SEP4 for an ensemble of 200 experiments for the case of the Exp distribution for an 16-floor setting. 


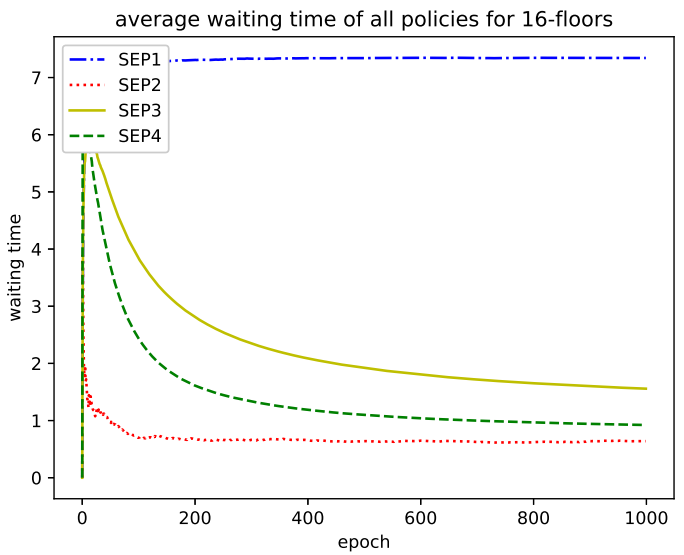

Figure A.6: The Average Waiting Time for SEP1, SEP2, SEP3 and SEP4 for an ensemble of 200 experiments for the case of the InvExp distribution for an 16-floor setting.

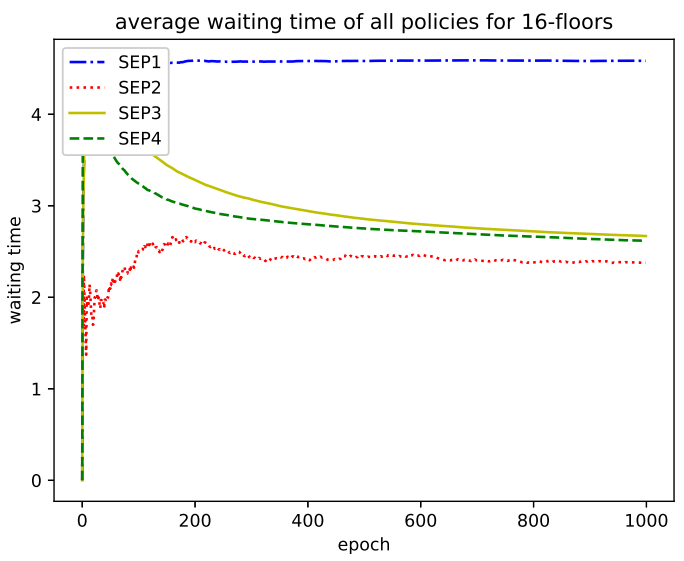

Figure A.7: The Average Waiting Time for SEP1, SEP2, SEP3 and SEP4 for an ensemble of 200 experiments for the case of the Gaussian distribution for an 16-floor setting. 


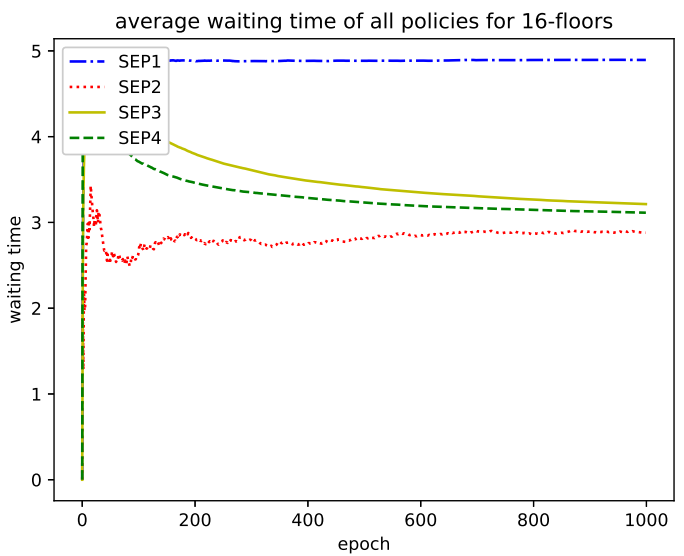

Figure A.8: The Average Waiting Time for SEP1, SEP2, SEP3 and SEP4 for an ensemble of 200 experiments for the case of the Bimodal distribution for an 16-floor setting.

Table A.3: Simulation results for the previous and newly proposed solutions for the SEP for an ensemble of 200 experiments for the 20 -floor settings. The results are given here as a tuple $(\alpha, \beta)$ where the first field, $\alpha$, is the best optimal parking floor and the second field, $\beta$ is the $A W T$ in terms of number of floors travelled for the elevator to reach the passenger from the parked location.

\begin{tabular}{|c|c|c|c|c|}
\hline Dist & SEP1 & SEP2 & SEP3 & SEP4 \\
\hline $\operatorname{Exp}$ & $(-, 9.342)$ & $(1,0.676)$ & $(2,1.840)$ & $(1,0.938)$ \\
\hline InvExp & $(-, 9.341)$ & $(20,0.723)$ & $(19,1.921)$ & $(20,0.976)$ \\
\hline Gaussian & $(-, 5.538)$ & $(11,2.453)$ & $(11,2.805)$ & $(10,2.774)$ \\
\hline Bimodal & $(-, 5.867)$ & $(13,3.261)$ & $(13,3.687)$ & $(13,3.639)$ \\
\hline
\end{tabular}




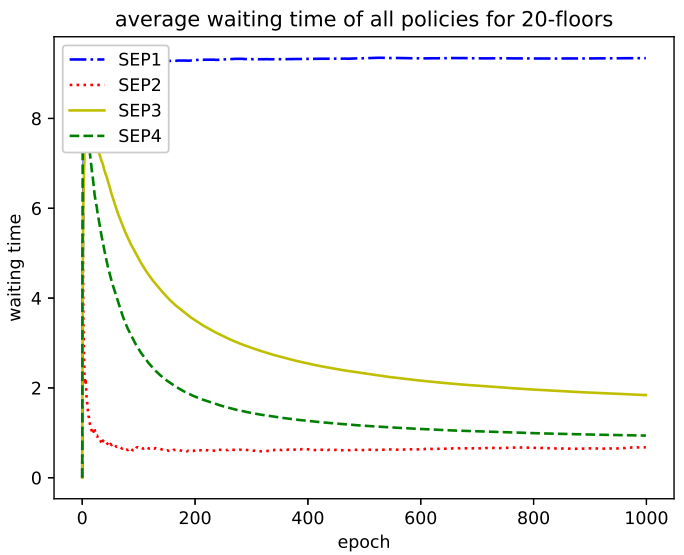

Figure A.9: The Average Waiting Time for SEP1, SEP2, SEP3 and SEP4 for an ensemble of 200 experiments for the case of the Exp distribution for an 20-floor setting.

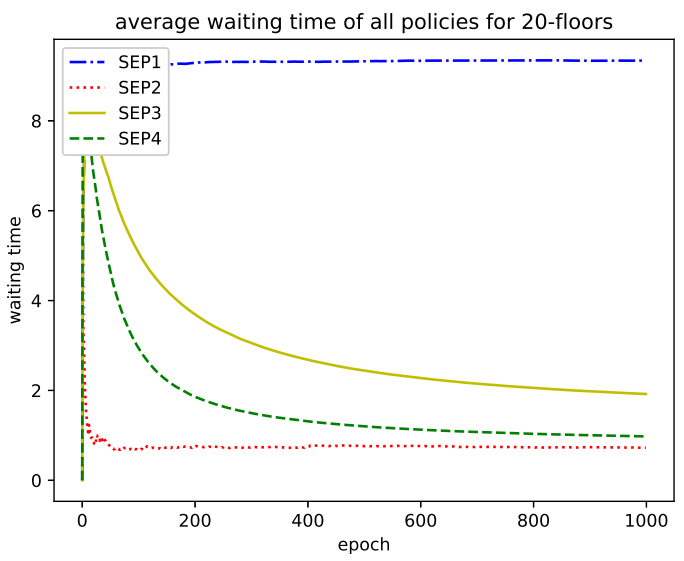

Figure A.10: The Average Waiting Time for SEP1, SEP2, SEP3 and SEP4 for an ensemble of 200 experiments for the case of the InvExp distribution for an 20-floor setting. 


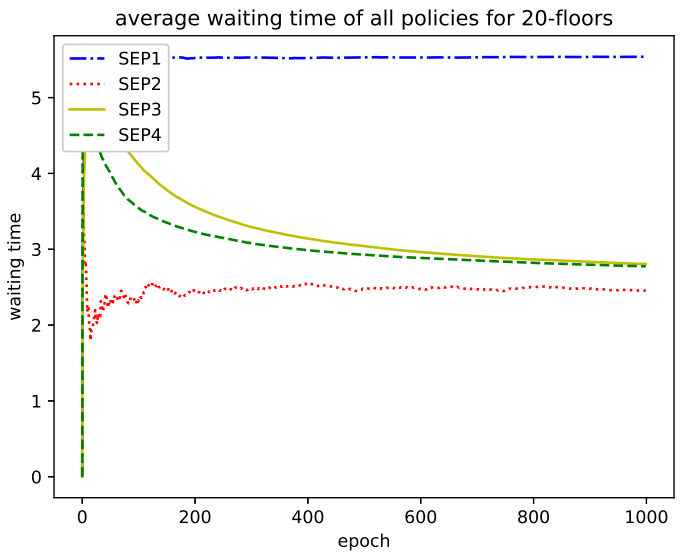

Figure A.11: The Average Waiting Time for SEP1, SEP2, SEP3 and SEP4 for an ensemble of 200 experiments for the case of the Gaussian distribution for an 20-floor setting.

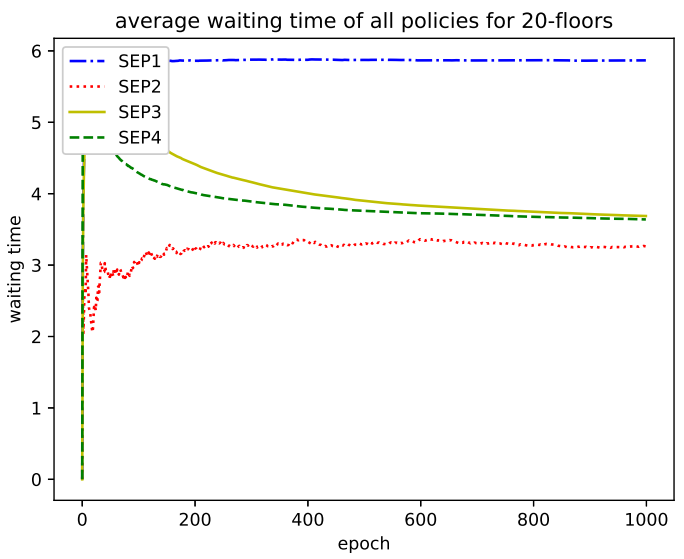

Figure A.12: The Average Waiting Time for SEP1, SEP2, SEP3 and SEP4 for an ensemble of 200 experiments for the case of the Bimodal distribution for an 20-floor setting. 


\section{Appendix B}

\section{MEP Results}

This appendix will include all the results and plots acquired from the simulations done for all the building settings, and for all the pertinent distributions for the case of the MEP. The notations, terminologies and legends are as described in Chapter 4.

Table B.1: Simulation results for the previous and newly proposed solutions for the MEP for an ensemble of 200 experiments for the 8-floor settings. The results are given here as a tuple $(\alpha, \beta)$ where the first field, $\alpha=\left\langle\alpha_{1}, \alpha_{2}\right\rangle$, is the best optimal parking floors for each elevator and the second field, $\beta$ is the $A W T$ in terms of number of floors travelled for the elevator to reach the passenger from the parked locations.

\begin{tabular}{c|cccc|}
\hline Dist & MEP1 & MEP2 & MEP3 & MEP4 \\
\hline Exp & $(-, 3.3608)$ & $(\langle 1,3\rangle, 0.3491)$ & $(\langle 1,2\rangle, 0.4314)$ & $(\langle 1,2\rangle, 0.3693)$ \\
InvExp & $(-, 3.3615)$ & $(\langle 6,8\rangle, 0.3513)$ & $(\langle 7,8\rangle, 0.4282)$ & $(\langle 7,8\rangle, 0.4634)$ \\
Gaussian & $(-, 1.7348)$ & $(\langle 2,6\rangle, 0.9823)$ & $(\langle 4,5\rangle, 1.2354)$ & $(\langle 4,6\rangle, 1.2342)$ \\
Bimodal & $(-, 1.7990)$ & $(\langle 3,6\rangle, 0.6504)$ & $(\langle 3,6\rangle, 0.9992)$ & $(\langle 5,6\rangle, 0.8838)$ \\
\hline
\end{tabular}




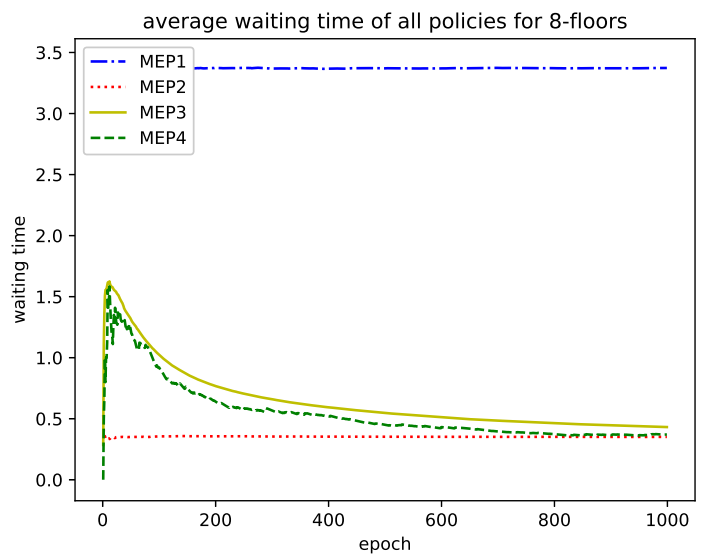

Figure B.1: The Average Waiting Time for MEP1, MEP2, MEP3 and MEP4 for an ensemble of 200 experiments for the case of the Exp distribution for an 8-floor setting.

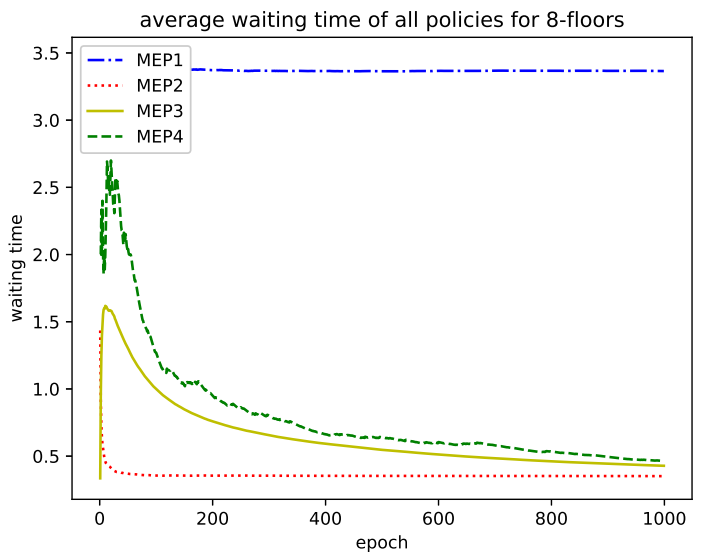

Figure B.2: The Average Waiting Time for MEP1, MEP2, MEP3 and MEP4 for an ensemble of 200 experiments for the case of the InvExp distribution for an 8-floor setting. The graph for MEP3 showed a superior performance to MEP4, and this was probably a statistical anomaly. 


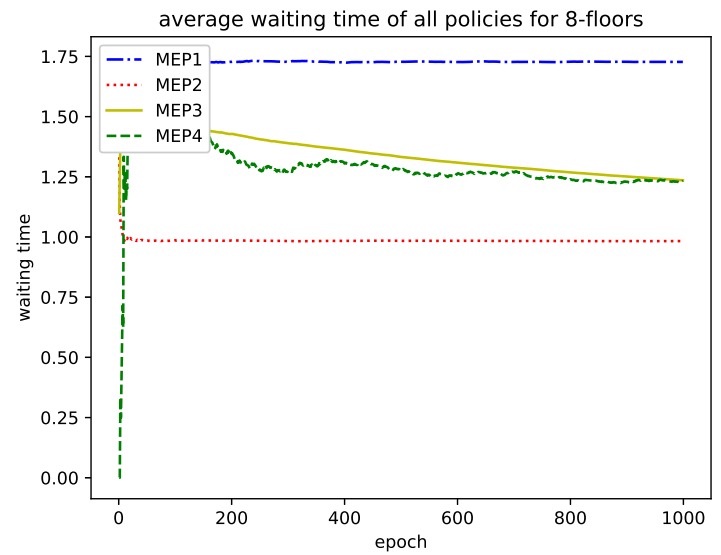

Figure B.3: The Average Waiting Time for MEP1, MEP2, MEP3 and MEP4 for an ensemble of 200 experiments for the case of the Gaussian distribution for an 8-floor setting.

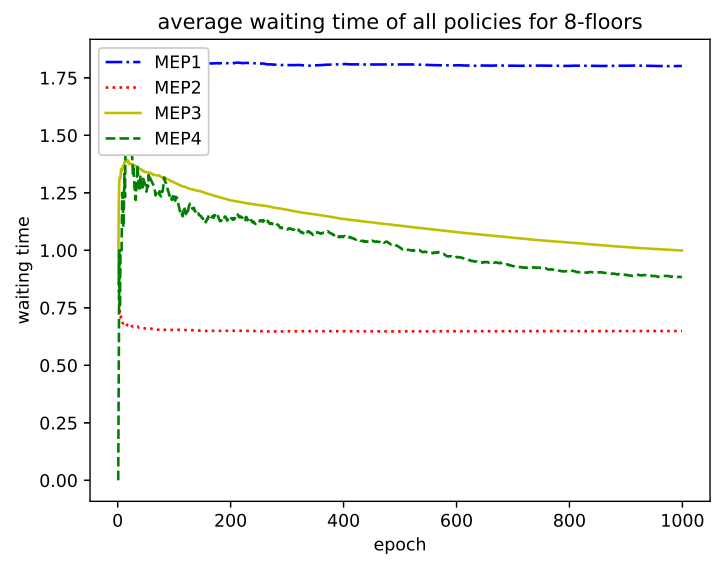

Figure B.4: The Average Waiting Time for MEP1, MEP2, MEP3 and MEP4 for an ensemble of 200 experiments for the case of the Bimodal distribution for an 8-floor setting. 
Table B.2: Simulation results for the previous and newly proposed solutions for the MEP for an ensemble of 200 experiments for the 16-floor settings. The results are given here as a tuple $(\alpha, \beta)$ where the first field, $\alpha=\left\langle\alpha_{1}, \alpha_{2}, \alpha_{3}\right\rangle$, is the best optimal parking floors for each elevator and the second field, $\beta$ is the $A W T$ in terms of number of floors travelled for the elevator to reach the passenger from the parked locations.

\begin{tabular}{c|cccc|}
\hline Dist & MEP1 & MEP2 & MEP3 & MEP4 \\
\hline Exp & $(-, 7.347)$ & $(\langle 1,3,5\rangle, 0.3023)$ & $(\langle 1,2,3\rangle, 0.381)$ & $(\langle 1,2,2\rangle, 0.362)$ \\
InvExp & $(-, 7.341)$ & $(\langle 12,14,16\rangle, 0.301)$ & $(\langle 15,16,16\rangle, 0.390)$ & $(\langle 15,16,16\rangle, 0.329)$ \\
Gaussian & $(-, 3.175)$ & $(\langle 5,9,11\rangle, 1.048)$ & $(\langle 7,9,10\rangle, 1.446)$ & $(\langle 7,9,10\rangle, 1.475)$ \\
Bimodal & $(-, 3.304)$ & $(\langle 5,8,12\rangle, 1.075)$ & $(\langle 6,12,13\rangle, 1.671)$ & $(\langle 10,12,13\rangle, 1.436)$ \\
\hline
\end{tabular}

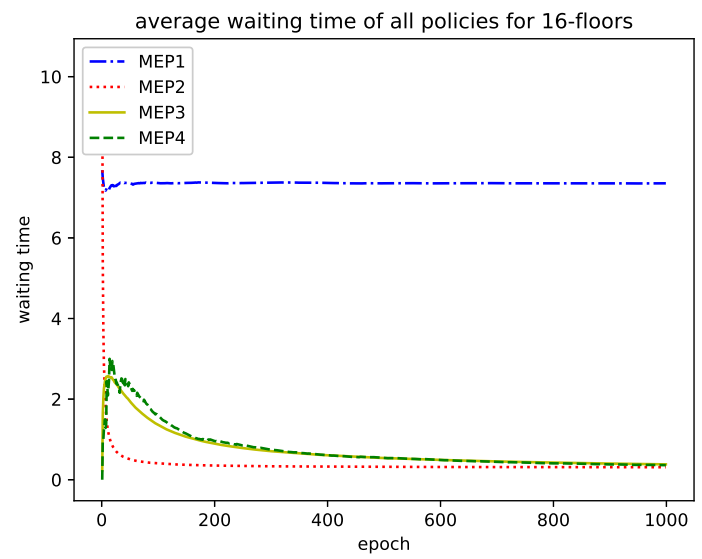

Figure B.5: The Average Waiting Time for MEP1, MEP2, MEP3 and MEP4 for an ensemble of 200 experiments for the case of the Exp distribution for an 16-floor setting.

Table B.3: Simulation results for the previous and newly proposed solutions for the MEP for an ensemble of 200 experiments for the 20-floor settings. The results are given here as a tuple $(\alpha, \beta)$ where the first field, $\alpha=\left\langle\alpha_{1}, \alpha_{2}, \alpha_{3}\right\rangle$, is the best optimal parking floors for each elevator and the second field, $\beta$ is the $A W T$ in terms of number of floors travelled for the elevator to reach the passenger from the parked locations.

\begin{tabular}{c|cccc|}
\hline Dist & MEP1 & MEP2 & MEP3 & MEP4 \\
\hline Exp & $(-, 9.345)$ & $(\langle 1,3,5\rangle, 0.306)$ & $(\langle 1,2,3\rangle, 0.418)$ & $(\langle 1,2,2\rangle, 0.442)$ \\
InvExp & $(-, 9.340)$ & $(\langle 16,18,20\rangle, 0.300)$ & $(\langle 19,20,20\rangle, 0.419)$ & $(\langle 19,19,20\rangle, 0.481)$ \\
Gaussian & $(-, 4.231)$ & $(\langle 7,11,13\rangle, 1.076)$ & $(\langle 9,10,12\rangle, 1.491)$ & $(\langle 9,11,12\rangle, 1.408)$ \\
Bimodal & $(-, 3.959)$ & $(\langle 7,13,15\rangle, 1.108)$ & $(\langle 7,14,15\rangle, 1.842)$ & $(\langle 13,15,15\rangle, 1.735)$ \\
\hline
\end{tabular}




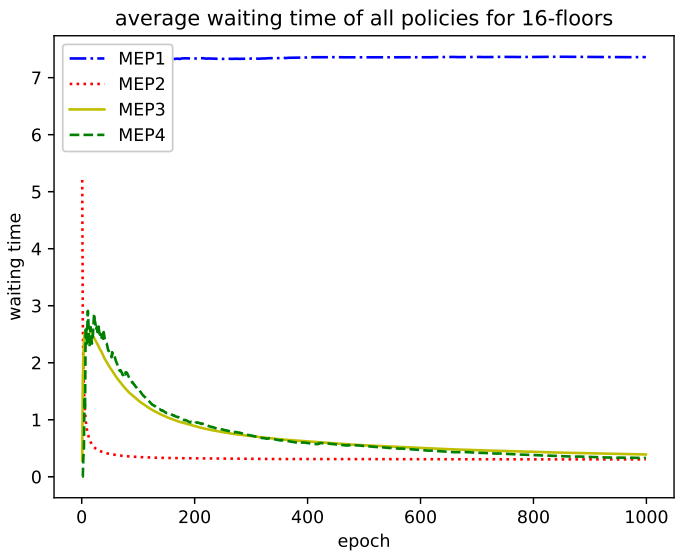

Figure B.6: The Average Waiting Time for MEP1, MEP2, MEP3 and MEP4 for an ensemble of 200 experiments for the case of the InvExp distribution for an 16-floor setting.

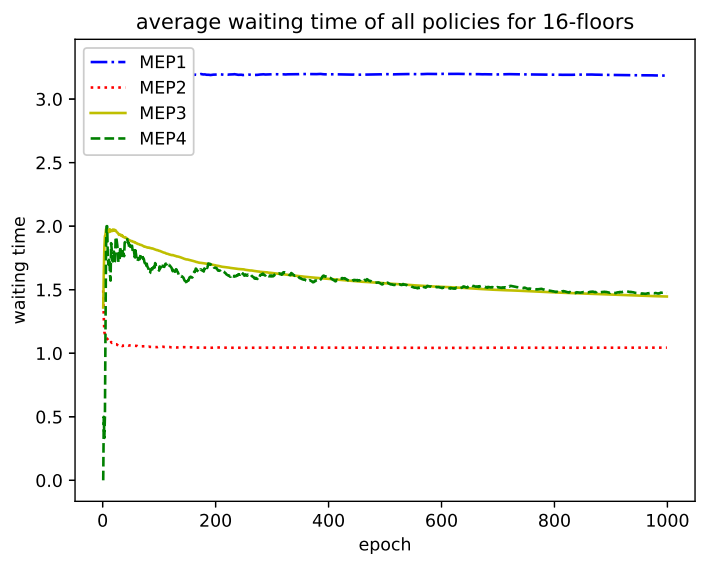

Figure B.7: The Average Waiting Time for MEP1, MEP2, MEP3 and MEP4 for an ensemble of 200 experiments for the case of the Gaussian distribution for an 16-floor setting. 


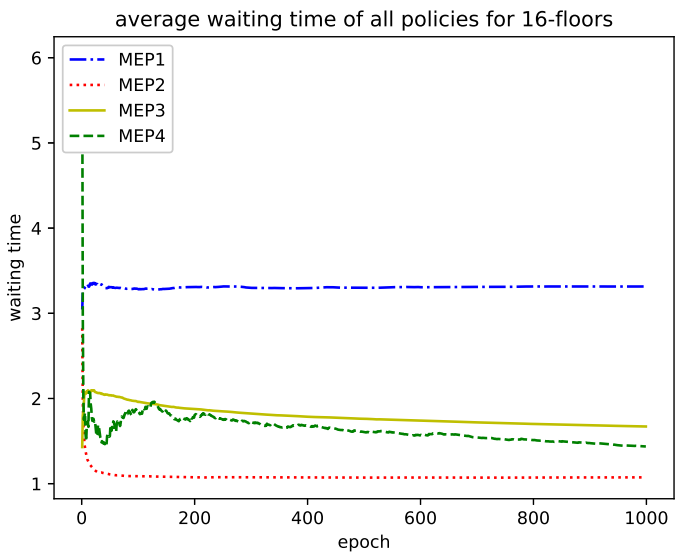

Figure B.8: The Average Waiting Time for MEP1, MEP2, MEP3 and MEP4 for an ensemble of 200 experiments for the case of the Bimodal distribution for an 16-floor setting.

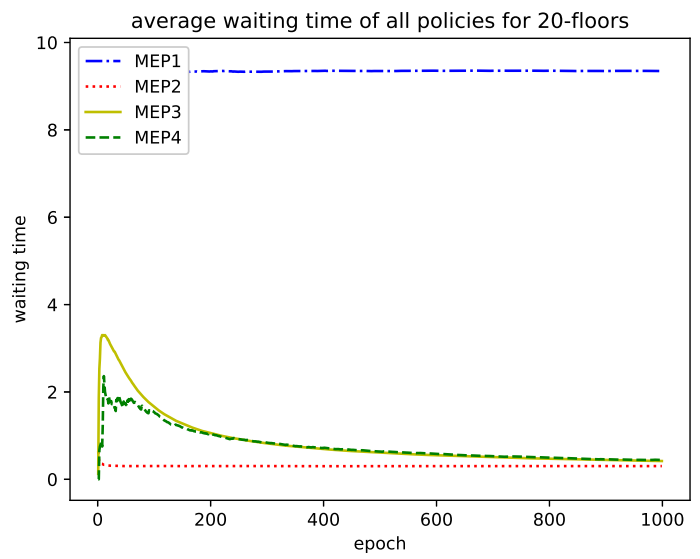

Figure B.9: The Average Waiting Time for MEP1, MEP2, MEP3 and MEP4 for an ensemble of 200 experiments for the case of the Exp distribution for an 20-floor setting. 


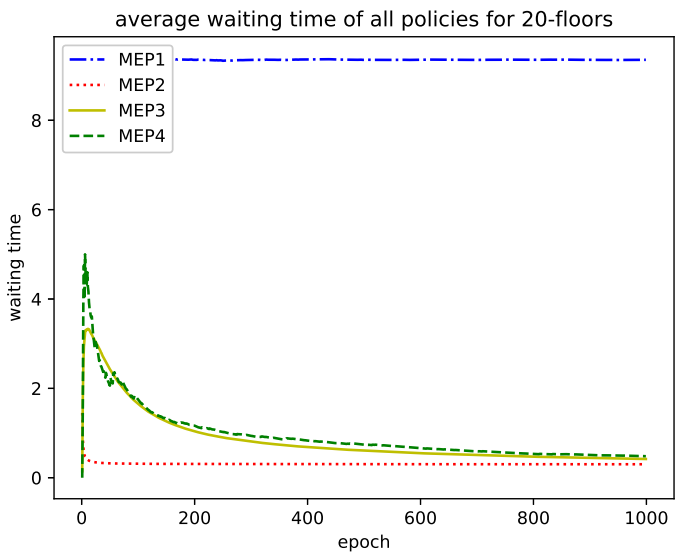

Figure B.10: The Average Waiting Time for MEP1, MEP2, MEP3 and MEP4 for an ensemble of 200 experiments for the case of the InvExp distribution for an 20-floor setting.

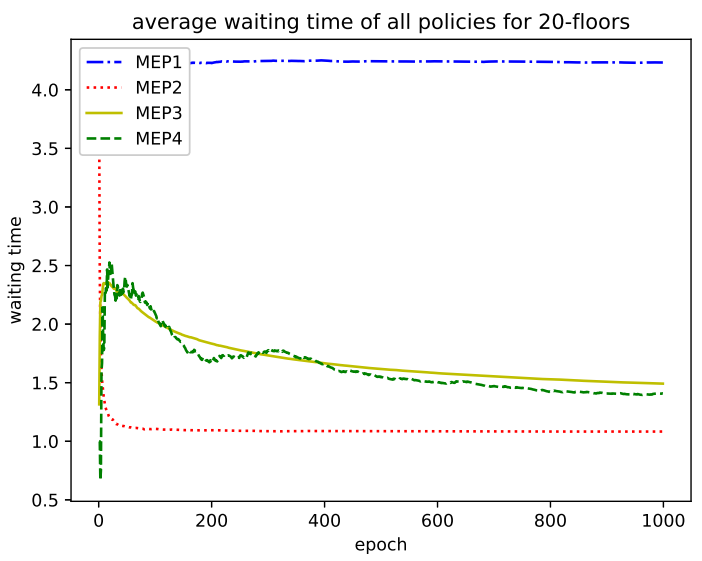

Figure B.11: The Average Waiting Time for MEP1, MEP2, MEP3 and MEP4 for an ensemble of 200 experiments for the case of the Gaussian distribution for an 20-floor setting. 


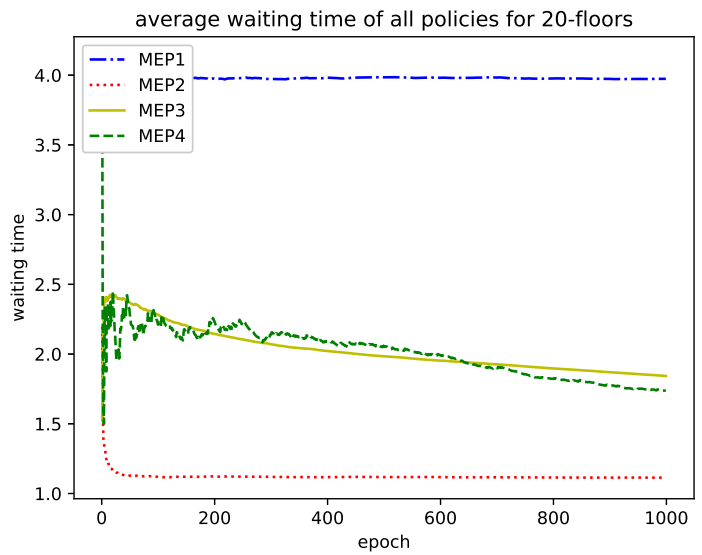

Figure B.12: The Average Waiting Time for MEP1, MEP2, MEP3 and MEP4 for an ensemble of 200 experiments for the case of the Bimodal distribution for an 20-floor setting. 


\section{Bibliography}

[1] N Baba and Y Sawaragi. On the learning behavior of stochastic automata under a nonstationary random environment. IEEE Transactions on Systems Man and Cybernetics, 2(SMC-5):273-275, 1975.

[2] A Barto and R H Crites. Improving elevator performance using reinforcement learning. Proceedings of the 8th International Conference on Neural Information Processing Systems, pages 1017-1023, 1995.

[3] Berna Bolat and Pablo Cortés. Genetic and tabu search approaches for optimizing the hall call - Car allocation problem in elevator group systems. Applied Soft Computing Journal, 11(2):1792-1800, 2011.

[4] M. Brand and D. Nikovski. Optimal parking in group elevator control. IEEE International Conference on Robotics and Automation, 2004. Proceedings. ICRA '04. 2004, pages 1002-1008 Vol.1, 2004.

[5] Young Cheol Cho, Zavarin Gagov, and Wook Hyun Kwon. Elevator Group Control with Accurate Estimation of Hall Call Waiting Times. IEEE: International Conference on Robotics \&3 Automation, (May):447-452, 1999.

[6] J. Fernández, P. Cortés, J. Munuzuri, and J. Guadix. Dynamic fuzzy logic elevator group control system with relative waiting time consideration. IEEE Transactions on Industrial Electronics, 61(9):4912-4919, 2014.

[7] J Kabudian, M R Meybodi, and M M Homayounpour. Applying continuous action reinforcement learning automata (carla) to global training of hidden markov 
models. In Information Technology: Coding and Computing, 2004. Proceedings. ITCC 2004. International Conference on, volume 2, pages 638-642. IEEE, 2004.

[8] V Y Krylov. One stochastic automaton which is asymptotically optimal in random medium. Automation and Remote Control, 24:1114-1116, 1964.

[9] Yaowu Liu, Zhangyong Hu, Qiang Su, and Jiazhen Huo. Energy saving of elevator group control based on optimal zoning strategy with interfloor traffic. Proceedings - 3rd International Conference on Information Management, Innovation Management and Industrial Engineering, ICIII 2010, 3:328-331, 2010.

[10] G McMurtry and K Fu. A variable structure automaton used as a multimodal searching technique. IEEE Transactions on Automatic Control, 11(3):379-387, 1966.

[11] M R Meybodi and H Beigy. New learning automata based algorithms for adaptation of backpropagation algorithm parameters. International Journal of Neural Systems, 12(01):45-67, 2002.

[12] S Misra and B J Oommen. GPSPA: A new adaptive algorithm for maintaining shortest path routing trees in stochastic networks. International Journal of Communication Systems, 17(10):963-984, 2004.

[13] S Molina and G Leguizam. An ACO Model for a Non-stationary Formulation of the Single Elevator Problem on. 7(1):45-51, 2007.

[14] K S Narendra and M A L Thathachar. Learning Automata: A Survey. IEEE Transactions on systems, man, and cybernetics, 4(4):323-334, 1974.

[15] K. S. Narendra and M. A. L. Thathachar. Learning automata: an introduction. Courier Corporation, 2012.

[16] K S Narendra and R Viswanathan. A two-level system of stochastic automata for periodic random environments. In Decision and Control, 1971 IEEE Conference on, pages 234-239. IEEE, 1971. 
[17] Daniel Nikovski and Matthew Brand. Decision-theoretic group elevator scheduling. in 13th International Conference on Automated Planning and Scheduling, pages 133-142, 2003.

[18] M S Obaidat, G I Papadimitriou, A S Pomportsis, and H S Laskaridis. Learning automata-based bus arbitration for shared-medium ATM switches. IEEE Transactions on Systems, Man, and Cybernetics, Part B (Cybernetics), 32(6):815-820, 2002.

[19] B J Oommen and J K Lanctot. Discretized pursuit learning automata. Systems, Man and Cybernetics, IEEE Transactions on, 20(4):931-938, 1990.

[20] B J Oommen and T D Roberts. Continuous learning automata solutions to the capacity assignment problem. IEEE Transactions on Computers, 49(6):608-620, 2000 .

[21] G I Papadimitriou and Pomportsis A S. Learning-automata-based TDMA protocols for broadcast communication systems with bursty traffic. IEEE Communications Letters, 4(3):107-109, 2000.

[22] Mahmut Parlar, Moosa Sharafali, and Jihong Ou. Optimal parking of idle elevators under myopic and state-dependent policies. European Journal of Operational Research, 170(3):863-886, 2006.

[23] Steven Porretta, Michel Barbeau, and Joaquin Garcia-alfaro. Learning to Communicate Underwater An Exploration of Limited Mobility Agents. pages 4-8, 2017.

[24] A S Poznyak. Learning automata in stochastic programming problems. Automat. Telemeh, 10:84-96, 1973.

[25] F Seredyński. Distributed scheduling using simple learning machines. European Journal of Operational Research, 107(2):401-413, 1998. 
[26] I J Shapiro and K S Narendra. Use of stochastic automata for parameter selfoptimization with multimodal performance criteria. IEEE Transactions on Systems Science and Cybernetics, 5(4):352-360, 1969.

[27] Clebes A Silva, Marcela N Oliveira, and Wesley P Calixto. Simulation and minimization of waiting time in rows of elevators of public buildings.

[28] Jin Sun, Qianchuan Zhao, Peter B. Luh, and Mauro J. Atalla. Estimation of optimal elevator scheduling performance. Proceedings - IEEE International Conference on Robotics and Automation, 2006(May):1078-1083, 2006.

[29] Shunji Tanaka, Yasuyuki Innami, and Mituhiko Araki. A study on objective functions for dynamic operation optimization of a single-car elevator system with destination hall call registration. Conference Proceedings - IEEE International Conference on Systems, Man and Cybernetics, 7:6274-6279, 2004.

[30] Shunji Tanaka, Yukihiro Uraguchi, and Mituhiko Araki. Dynamic optimization of the operation of single-car elevator systems with destination hall call registration: Part I. Formulation and simulations. European Journal of Operational Research, 167(2):550-573, 2005.

[31] Shunji Tanaka, Yukihiro Uraguchi, and Mituhiko Araki. Dynamic optimization of the operation of single-car elevator systems with destination hall call registration: Part II. The solution algorithm. European Journal of Operational Research, 167(2):550-573, 2005.

[32] Emre Oner Tartan, Hamit Erdem, and Ali Berkol. Optimization of waiting and journey time in group elevator system using genetic algorithm. In INISTA 2014 - IEEE International Symposium on Innovations in Intelligent Systems and Applications, Proceedings, pages 361-367, 2014.

[33] M A L Thathachar and K Ramakrishnan. A hierarchical system of learning automata. IEEE Transactions On Systems Man And Cybernetics, 11(3):236$241,1981$. 
[34] M A L Thathachar and P S Sastry. A class of rapidly converging algorithms for learning automata. In IEEE Int. Conf. on Systems, Man and Cybernatics. IEEE, 1984.

[35] M A L Thathachar and P S Sastry. A new approach to the design of reinforcement schemes for learning automata. IEEE Transactions on Systems, Man, and Cybernetics, SCM-15(1):168-175, 1985.

[36] M A L Thathachar and P S Sastry. Estimator algorithms for learning automata. In Platinum Jubilee Conference on Systems and Signal Processing, 1986.

[37] M Tsetlin. On behaviour of finite automata in random medium. Avtomat. $i$ Telemekh, 22(10):1345-1354, 1961.

[38] V Varshavskii and I P Vorontsova. On the behavior of stochastic automata with a variable structure. Avtomatika i Telemekhanika, 24(3):353-360, 1963.

[39] Jingyang Xu and Tianke Feng. Single Elevator Scheduling Problem with Complete Information: An Exact Model using Mixed Integer Linear Programming. pages 2894-2899, 2016.

[40] E Zerz, C L Beck, J C Doyle, A Megretski, J Shamma, P Cabinet, A Nemirovskii, A J Laub, and M Chilali. Exact Calculation of Expected Waiting Times for Group Elevator Control. 49(10):2002-2005, 2004.

[41] Liu Zheng, Shu Guang, and Dong Hui. Research of elevator group scheduling system based on reinforcement learning algorithm. Measurement, Information and Control (ICMIC), 2013 International Conference on, 01:606-610, 2013.

[42] Qun Zong, Weijia Wang, and Chaofeng Song. On Elevator Dynamic Scheduling Policy during Up-peak Traffic Based on Reinforcement Learning Algorithm. Proceedings of the International Conference on Sensing, Computing and Automation, (3):434-438, 2006. 